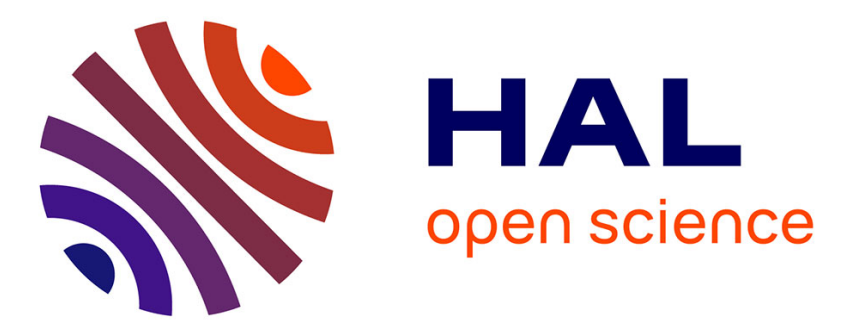

\title{
Where does the Thermospheric Ionospheric GEospheric Research (TIGER) Program go?
}

Gerhard Schmidtke, S.V. Avakyan, J. Berdermann, V. Bothmer, G. Cessateur, L. Ciraolo, L. Didkovsky, Thierry Dudok de Wit, F.G. Eparvier, A. Gottwald, et al.

\section{To cite this version:}

Gerhard Schmidtke, S.V. Avakyan, J. Berdermann, V. Bothmer, G. Cessateur, et al.. Where does the Thermospheric Ionospheric GEospheric Research (TIGER) Program go?. Advances in Space Research, 2015, 56 (8), pp.1547-1577. 10.1016/j.asr.2015.07.043 . insu-01183469

\section{HAL Id: insu-01183469 \\ https://hal-insu.archives-ouvertes.fr/insu-01183469}

Submitted on 31 May 2016

HAL is a multi-disciplinary open access archive for the deposit and dissemination of scientific research documents, whether they are published or not. The documents may come from teaching and research institutions in France or abroad, or from public or private research centers.
L'archive ouverte pluridisciplinaire HAL, est destinée au dépôt et à la diffusion de documents scientifiques de niveau recherche, publiés ou non, émanant des établissements d'enseignement et de recherche français ou étrangers, des laboratoires publics ou privés.

\section{(1) $(1) \Theta$}

Distributed under a Creative Commons Attribution - NonCommercial - NoDerivatives| 4.0 


\section{Accepted Manuscript}

Review

Where does the Thermospheric Ionospheric GEospheric Research (TIGER)

Program go?

G. Schmidtke, S.V. Avakyan, J. Berdermann, V. Bothmer, G. Cessateur, L. Ciraolo, L. Didkovsky, T. Dudok de Wit, F.G. Eparvier, A. Gottwald, M.

Haberreiter, R. Hammer, Ch. Jacobi, N. Jakowski, M. Kretzschmar, J. Lilensten, M. Pfeifer, S.M. Radicella, R. Schäfer, W. Schmidt, S.C. Solomon, G. Thuillier, W.K. Tobiska, S. Wieman, T.N. Woods

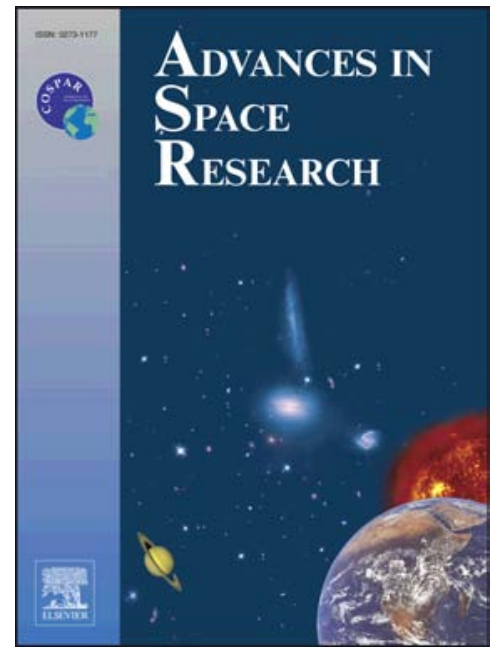

PII:

S0273-1177(15)00562-1

DOI: http://dx.doi.org/10.1016/j.asr.2015.07.043

Reference: JASR 12376

To appear in: Advances in Space Research

Received Date:

27 May 2015

Revised Date: 29 July 2015

Accepted Date:

30 July 2015

Please cite this article as: Schmidtke, G., Avakyan, S.V., Berdermann, J., Bothmer, V., Cessateur, G., Ciraolo, L., Didkovsky, L., Dudok de Wit, T., Eparvier, F.G., Gottwald, A., Haberreiter, M., Hammer, R., Jacobi, Ch., Jakowski, N., Kretzschmar, M., Lilensten, J., Pfeifer, M., Radicella, S.M., Schäfer, R., Schmidt, W., Solomon, S.C., Thuillier, G., Tobiska, W.K., Wieman, S., Woods, T.N., Where does the Thermospheric Ionospheric GEospheric Research (TIGER) Program go?, Advances in Space Research (2015), doi: http://dx.doi.org/10.1016/j.asr.2015.07.043

This is a PDF file of an unedited manuscript that has been accepted for publication. As a service to our customers we are providing this early version of the manuscript. The manuscript will undergo copyediting, typesetting, and review of the resulting proof before it is published in its final form. Please note that during the production process errors may be discovered which could affect the content, and all legal disclaimers that apply to the journal pertain. 


\title{
Where does the Thermospheric Ionospheric GEospheric Research (TIGER) Program go?
}

${ }^{*}$ G. Schmidtke ${ }^{\mathrm{a}}$, S. V. Avakyan ${ }^{\mathrm{j}}$, J. Berdermann ${ }^{\mathrm{d}}$, V. Bothmer ${ }^{\mathrm{p}}$, G. Cessateur ${ }^{\mathrm{h}, \mathrm{o}}$, L. Ciraolo ${ }^{\mathrm{g}}$, L. Didkovsky ${ }^{\mathrm{r}}$, T. Dudok de Wit ${ }^{\mathrm{m}}$, F. G. Eparvier ${ }^{\mathrm{b}}$, A. Gottwald ${ }^{\mathrm{q}}$, M. Haberreiter ${ }^{\mathrm{h}}$, R. Hammer $^{\mathrm{k}}$, Ch. Jacobi ${ }^{\mathrm{c}}$, N. Jakowski ${ }^{\mathrm{d}}$, M. Kretzschmar ${ }^{\mathrm{m}}$, J. Lilensten ${ }^{1}$, M. Pfeifer ${ }^{\mathrm{a}}$, S. M. Radicella $^{\mathrm{g}}$, R. Schäfer ${ }^{\mathrm{a}}$, W. Schmidt ${ }^{\mathrm{k}}$, S. C. Solomon ${ }^{\mathrm{i}}$, G. Thuillier ${ }^{\mathrm{e}}$, W. K. Tobiska ${ }^{\mathrm{f}}, \mathrm{S}$. Wieman $^{\mathrm{r}}$, T. N. Woods ${ }^{\mathrm{b}}$,

${ }^{a}$ Fraunhofer IPM, Heidenhofstrasse 8, D-79110 Freiburg, Germany,

${ }^{\mathrm{b}}$ LASP, University of Colorado, Boulder, CO 80303, USA,

'Universität Leipzig, Germany,

${ }^{\mathrm{d}}$ ICN-DLR, Neustrelitz, Germany,

${ }^{\mathrm{e}}$ LATMOS-CNRS, Guyancourt, France,

${ }^{\mathrm{f}}$ Space Environment, Technologies, Pacific Palisades, CA 90272, USA,

${ }^{\mathrm{g}}$ ICTP, Trieste, Italy,

${ }^{\mathrm{h}}$ Physikalisch-Meteorologisches Observatorium Davos/Word Radiation Center, Davos, Switzerland,

${ }^{\mathrm{i}} \mathrm{HAO} / \mathrm{NCAR}$, Boulder, CO 80307, USA

${ }^{j}$ Vavilov State Optical Institute, St. Petersburg State Politechnical University, Russia,

${ }^{\mathrm{k}}$ Kiepenheuer-Institut für Sonnenphysik, Freiburg i. Br., Germany,

${ }^{1}$ LPG, CNRS and Joseph Fourier University, Saint-Martin d'Hères, France,

${ }^{\mathrm{m}}$ LPC2E, CNRS and University of Orléans, Orléans, France,

${ }^{\circ}$ Belgian Institute for Space Aeronomy (BIRA-IASB), Belgium,

${ }^{\mathrm{P}}$ Georg-August-Universität Göttingen, Germany,

${ }^{\mathrm{q}}$ Physikalisch-Technische Bundesanstalt, Berlin, Germany,

${ }^{\mathrm{r}} \mathrm{UCLA}$, Los Angeles, USA

Key words: UV/EUV solar spectral irradiance - instrumentation - calibration - modeling

\begin{abstract}
At the $10^{\text {th }}$ Thermospheric Ionospheric GEospheric Research (TIGER/COSPAR) symposium held in Moscow in 2014 the achievements from the start of TIGER in 1998 were summarized. During that period, great progress was made in measuring, understanding, and modeling the highly variable UV-Soft X-ray (XUV) solar spectral irradiance (SSI), and its effects on the *Corresponing author. Tel.: +49 7618857 176; fax: +49 7618857224.

E-Mail address: gerhard.schmidtke@ipm.fraunhofer.de (G. Schmidtke).
\end{abstract}


upper atmosphere. However, after more than 50 years of work the radiometric accuracy of SSI observation is still an issue and requires further improvement. Based on the extreme ultraviolet (EUV) data from the SOLAR/SolACES, and SDO/EVE instruments, we present a combined data set for the spectral range from 16.5 to $105.5 \mathrm{~nm}$ covering a period of 3.5 years from 2011 through mid of 2014. This data set is used in ionospheric modeling of the global total electron content (TEC), and in validating EUV SSI modeling. For further investigations the period of 3.5 years is being extended to about 12 years by including data from SOHO/SEM and TIMED/SEE instruments. Similarly, UV data are used in modeling activities. After summarizing the results, concepts are proposed for future real-time SSI measurements with in-flight calibration as experienced with the ISS SOLAR payload, for the development of a space weather camera for observing and investigating space weather phenomena in real-time, and for providing data sets for SSI and climate modeling. Other planned topics are the investigation of the relationship between solar EUV/UV and visible/near-infrared emissions, the impact of X-rays on the upper atmosphere, the development of solar EUV/UV indices for different applications, and establishing a shared TIGER data system for EUV/UV SSI data distribution and real-time streaming, also taking into account the achievements of the FP7 SOLID (First European SOLar Irradiance Data Exploitation) project. - For further progress it is imperative that coordinating activities in this special field of solar-terrestrial relations and solar physics is emphasized.

\section{Goals of TIGER}

After more than three decades of activities for providing EUV/UV SSI data the TIGER Program was established within the framework of the SCOSTEP International Solar Cycle Study Working (ISCS) Group 1 in 1998. Since the 5th TIGER Symposium (Paris/France, 2004) these symposia are held as a session of COSPAR Scientific Assembly (Schmidtke et al., 2006a). The primary objective of this initiative was and still is to determine the variable solar spectral irradiance (SSI) in the extreme ultraviolet/ultraviolet (EUV/UV) and X-ray regions with the ultimate purpose to better understand solar-terrestrial relations including thermospheric-ionospheric (T/I) processes. This is also embedded in the investigation of space weather, and climate change requiring the compilation of long-term SSI data sets. In this context the TIGER/COSPAR topics and goals are:

- Continuous measurement, verification, and compilation of EUV/UV SSI data,

- Developing instrumentation and methods for making a radiometric standard for calibrating and cross-calibrating EUV/UV SSI measurements in space,

- Measurement of airglow/auroral EUV/UV radiation and developing instrumentation and missions for real-time monitoring of space weather phenomena,

- Investigation of relationships between solar EUV/UV and VIS/NIR emissions,

- Modeling solar EUV/UV SSI for different applications,

- Developing solar EUV/UV indices for different applications,

- Modeling the thermosphere/ionosphere with EUV SSI data, 
- Developing instrumentation and missions for EUV/UV SSI real-time and in-line measurements, and

- Investigation of the role of X-rays in the upper atmosphere.

Inter-comparing total solar irradiance (TSI) with solar spectral irradiance (SSI) data from the soft X-ray through the infrared spectral regions is self-evident also for application in climate modeling.

This ambitious program can only be realized by coordinating worldwide activities, resources, and experience, keeping in mind the specific difficulties in space-born EUV/UV technology, and including the strong effects of aging and/or degradation.

TIGER/COSPAR has become an useful platform for acquiring a better understanding of the EUV/UV SSI and its effects on the terrestrial upper atmosphere. The following sections describe the progress on the TIGER goals and explore future opportunities to further address these goals.

\section{EUV/UV SSI: Measurement requirements}

After the first recording of a solar ultraviolet spectrum (Baum et al., 1946) aboard a German V2 rocket intense exploration of the solar EUV/UV radiation started aboard rockets and satellites. Various parameters, such as the Sunspot number, the solar radio flux, provide a strong correlation with the ionospheric density changes on time-scales of the solar cycle, the solar rotation, as well as short-term events such as solar flares. In order to investigate these types of solar phenomena that are relevant for a better understanding of solar-terrestrial relations, satellites provide an excellent platform for scientific instrumentation devoted to investigate physical processes of the Sun and/or terrestrial processes controlled by the variable EUV/UV energy input from the Sun.

Depending on the science topics of interest the terrestrial upper atmosphere responds at different time scales. While resonant and fluorescent scattering is generated in real-time, most of the airglow emissions are produced by impacts of photo electrons with atmospheric particles in nearly real-time. Based on the current understanding, airglow studies require EUV SSI data at 0.2 to $2 \mathrm{~nm}$ spectral resolution and a cadence of minutes.

Global parameters like thermospheric and ionospheric densities and temperatures show a delay in the response to solar EUV/UV changes up to the order of two days (see below). Taking also into account the EUV/UV absorption processes which are discussed in detail below only a modest spectral resolution of the solar spectral irradiance from the total solar disk with medium temporal resolution is required for thermospheric-ionospheric modeling. The measurement requirements for the EUV/UV SSI are the following:

- Broad spectral coverage: The solar radiation shorter than about $320 \mathrm{~nm}$ is completely absorbed in the Earth's atmosphere. The solar radiation which is most important for the T/I layers above about $80 \mathrm{~km}$ include the soft X-ray (SXR or UUV: 0.1-10 $\mathrm{nm}$ ) and the extreme ultraviolet (EUV: 10-121 nm). The far ultraviolet (FUV: 122-200 nm) is 
absorbed in the mesosphere. The middle ultraviolet (MUV: 200-300 nm) and near ultraviolet (NUV: $300-400 \mathrm{~nm}$ ) are more important for middle atmosphere studies (e.g. ozone) and climate change studies.

- Spectral resolution: Absorption by ionization continua of molecular oxygen $\left(\mathrm{O}_{2}\right)$ and nitrogen $\left(\mathrm{N}_{2}\right)$ and atomic oxygen $(\mathrm{O})$ dominates the spectral region shorter than $60 \mathrm{~nm}$. Given the smooth wavelength-dependence of these continua a spectral resolution of about $5 \mathrm{~nm}$ suffices for the $3 \mathrm{~nm}$ to $60 \mathrm{~nm}$ spectral range. However, because of the higher spectral dependence of Auger electron absorption shorter than $3 \mathrm{~nm}$ and of rotation-vibration absorption bands in the interval $>60 \mathrm{~nm}$ with increasing wavelength, a spectral resolution of $1-2 \mathrm{~nm}$ is required to allocate major solar emissions to the absorbing species for wavelengths longer than $60 \mathrm{~nm}$. a $0.1 \mathrm{~nm}$ resolution is needed for the $0.1 \mathrm{~nm}$ to $3.1 \mathrm{~nm}$ range.

- Medium temporal resolution: This requirement is driven by the approach how the EUV SSI data are applied in T/I modeling. In order to provide daily T/I parameters, at least daily measurements are needed but higher cadence is desired to achieve precision below $1 \%$. This requirement can be met, for example, by averaging over six EUV spectral recordings per day. Just for special applications like investigating airglow effects driven by solar flares a cadence down to 1 minute could be of interest. For future application of EUV SSI data in navigation data processing a similar temporal resolution is expected.

- Modest accuracy: With a solar variability in the EUV of a factor of two, the absolute total uncertainty, or accuracy, of the SSI needs to be $20 \%$ or better. It is the aim of the TIGER/COSPAR initiative to improve the radiometric accuracy of EUV SSI data to be better than $5 \%$. - As the FUV variability is smaller, only about $10 \%$, the accuracy goal is $1-5 \%$ for this spectral range. Pre-flight calibrations with synchrotron radiation sources can achieve a total uncertainty of $2-5 \%$. In view of the efficiency changes of the instruments experienced in space, regular re-calibration (preferable once per week) is required either within the instrument itself or via cross-calibration from another instrument operating in space.

\section{3. $\mathbf{E U V / U V ~ i n s t r u m e n t s ~ i n ~ s p a c e ~}$}

This Section provides an overview of relevant EUV/UV instruments in space, reflecting the state of the art with respect to the requirements described in Section 2.

\subsection{Recent and on-going EUV measurements}

Space missions recording solar EUV emissions from 1946 until 2014 have been reviewed by Schmidtke (2015) showing that there is no coherent data set covering the full EUV spectral region from 5-105 $\mathrm{nm}$ as relevant for the T/I system over a full period of a solar cycle, yet. The NASA TIMED Solar EUV Experiment (SEE) is closest to meeting this need with its daily spectral measurement between $27 \mathrm{~nm}$ and $190 \mathrm{~nm}$ from 2002 to 2015. SEE observations at wavelengths shorter than $27 \mathrm{~nm}$ are broad-band measurements with inadequate spectral resolution. In the past century the problem of aging/degrading of instruments hampered the radiometric accuracy of the data so that it is very difficult to allocate uncertainties to the data. 
For example, discrepancies up to a factor of four are published for the solar fluxes below 25 $\mathrm{nm}$ recorded with broad-band silicon detectors deposited with thin metallic films (Solomon et al., 2001).

As compiled in Figure 1 the situation has improved since 2000 with respect to time coverage and accuracy. Now data sets from different experiments are available at a radiometric accuracy of about $10 \%$ exceeding a period of a solar cycle. Continuing the ambitious task of merging these different EUV SSI data sets into one consistent composite is important future work to recommend it as a reference data set for T/I modeling and other applications. The achievements of the FP7 SOLID (First European SOLar Irradiance Data Exploitation) project will be included in this effort.

Currently, real-time EUV SSI data over the full spectral range are not yet available. However, technology combining low-cost and sophisticated instrumentation and data evaluation algorithms have matured to a stage for in-orbit verification (see Section 6.8). Monitors of a few SXR and EUV bands aboard the NOAA GOES satellites and the NASA EUV Variability Experiment (EVE, Woods et al, 2010) onboard the Solar Dynamics Observatory (SDO) provide real-time data products that are applicable for space weather studies as well as for use in space weather operations. 


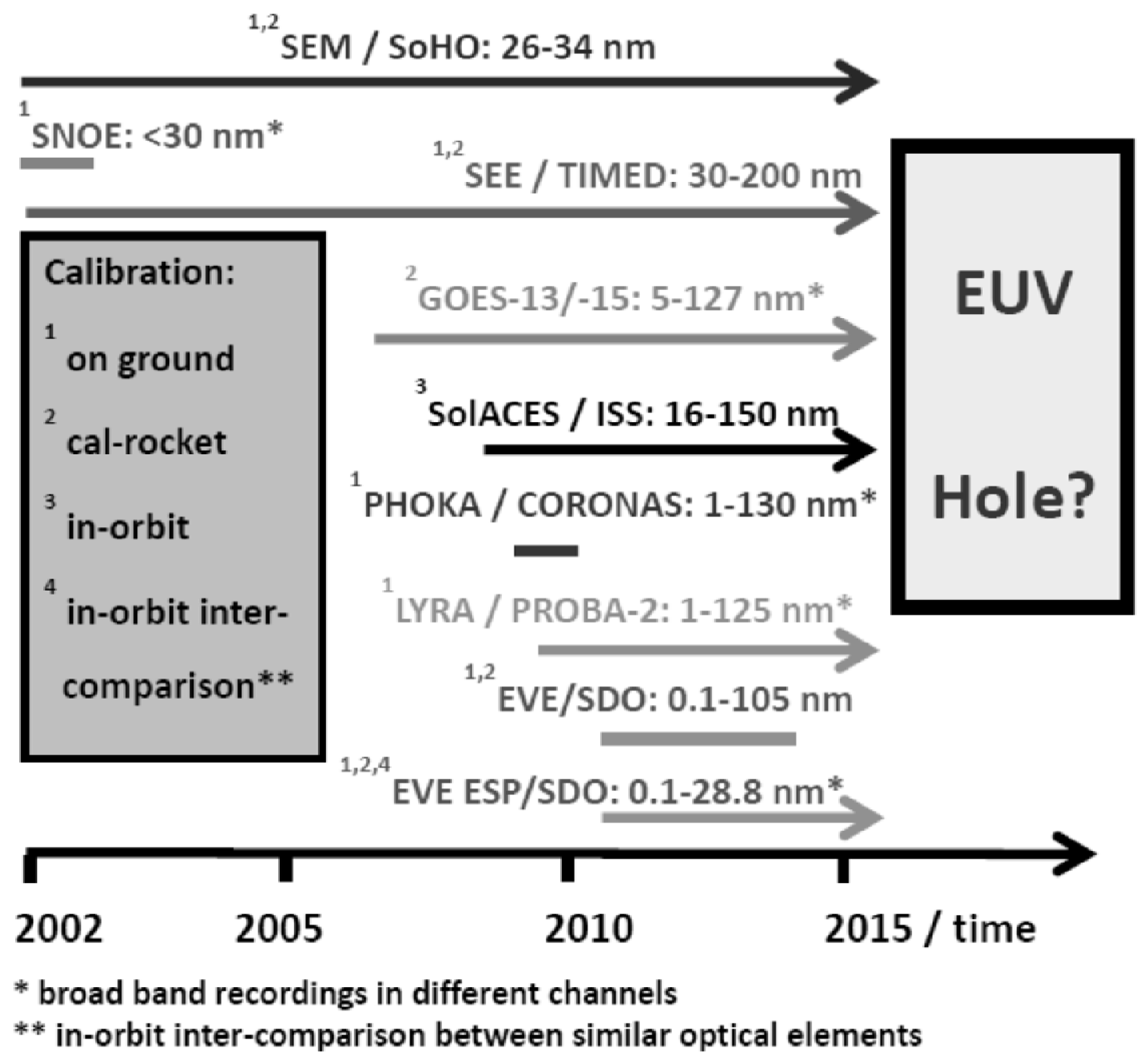

Figure 1: Compilation of recent and on-going EUV/UV SSI measurements.

Relevant missions are shortly characterized in the following sections:

\subsubsection{SOHO/SEM}

The SOHO/Solar EUV Monitor (SEM, Wieman et al., 2010) measures the solar flux in two band-passes: $26-34 \mathrm{~nm}\left(+/-1^{\text {st }}\right.$ order) and 0.1-50 nm (0-order) using a transmission grating and silicon photodiode detectors. The instrument has been providing nearly continuous EUV irradiance measurements for all of the $>22$ years SOHO has been in operation.

Calibration has been maintained based on sounding rocket under-flights - there is no in-flight calibration.

\subsubsection{TIMED/SEE}

Since the launch of the Thermosphere-Ionosphere-Mesosphere Energetics and Dynamics (TIMED) satellite in December, 2001 the Solar EUV Experiment (SEE) has been measuring the solar SSI EUV in the spectral range from about 27 to $190 \mathrm{~nm}$ with $0.4 \mathrm{~nm}$ spectral 
resolution and in broad bands between 0.1 to $30 \mathrm{~nm}$ (Woods et al., 2000; Woods et al., 2005; Woods and Eparvier, 2008; Woods, 2008). The wavelength range from 27-190 nm is measured with the EUV Grating Spectrograph (EGS), consisting of a normal-incidence concave reflection grating and a chevron stack of two microchannel plates with $64 \times 1024$ pixels (CODACON detector). The XUV detectors are thin foil films on silicon photodiodes. The SEE Solar Pointing Platform (SSPP) is a one-axis pointing platform as needed to make one solar observation per orbit with each observation lasting about 3 minutes. The TIMED orbit is nearly circular at about $615 \mathrm{~km}$ with an inclination of $74.1^{\circ}$, providing $15-16$ solar observations per day.

Calibration is performed on the ground, by redundant channels and a flat-field lamp in flight, by inter-calibration with calibration rockets flights once per year, and with inter-calibration with other space missions such as SDO/EVE (see below).

\subsubsection{GOES-13/-15}

GOES-13 and GOES-15 EUV-SSI measurements with the Extreme UltraViolet Sensor (EUVS) include broad-band observations in channels 5-15 nm, 25-34 nm, 42-63 nm, 17-81 $\mathrm{nm}$, and 118-127 nm since August, 2006 (Evans et al., 2010) using instruments similar in design to the SOHO/SEM. A next generation of EUVS and XRS for the GOES-R+ program with expanded capabilities is preparing for launch in 2016 (Eparvier et al., 2009; Chamberlin et al., 2009). The instrument EUVS optics consists of concave reflection gratings, thin freestanding foil filters, and arrays of silicon diodes. It is integrated aboard the American weather satellite in a geostationary orbit at a longitude of $75^{\circ}$ West with an orbital period of 24 hours. The earlier GOES satellites have included the X-Ray Sensor (XRS) that measures the SXR bands of 0.05-0.4 $\mathrm{nm}$ and 0.1-0.8 $\mathrm{nm}$, and these bands have been the reference for classifying X-ray flare magnitude since 1976. Previous XRS instruments have been ionization cells. For GOES-R the XRS will utilize silicon photodiode detectors.

Calibration is performed on ground and with inter-calibration with other space missions such as SDO/EVE and overlapping GOES missions (see below).

\subsubsection{SOLAR/SolACES}

Aboard the International Space Station (ISS) the Solar Auto-Calibrating EUV Spectrometers (SolACES) has been measuring SSI EUV data in the spectral range from 16 to $150 \mathrm{~nm}$ since February, 2008. The mission is extended to February, 2017. The spectrometers consist of planar gratings, parabolic mirrors, and channeltron detectors. In-flight calibration is achieved using three-current ionization chambers (IC) repeatedly re-filled with neon $(\mathrm{Ne})$, xenon $(\mathrm{Xe})$, and a mixture of Xe and nitric oxide (NO). The transmission of a series of filters overlapping the spectral range of interest is determined on demand using a filter wheel for positioning them on spectrometers and ionization chambers and vice versa (Schmidtke et al., 2006b; Schmidtke et al., 2014). The ISS orbits at a 51.6-degree inclination around the Earth with an altitude between 370 and 460 kilometers (230-286 miles) above Earth's surface. The orbit and the geometry of the ISS imply periodic gaps of two to three weeks of measurements with additional interruptions due to the orbit and to logistical events. There are 16 sunrises and 16 sunsets every day providing 20 minute periods of measurements during Solar Viewing 
Windows, each. It should be noted that the SolACES instrument is thermally stabilized minimizing efficiency changes caused by temperature changes. The electrical power feeding the thermal control system is well separated from the instrumental power line.

In-flight calibration primarily relies on the stability of the absorption cross-sections of inert gases because they do not change at all. IC measurements are performed whenever desired.

Based on the successful verification of the auto-calibrating method SolACES is considered as, to be the next generation instrumentation for solar XUV/EUV measurements.

\subsubsection{PROBA-2/LYRA}

The Large Yield Radiometer (LYRA, Hochedez et al., 2006) was launched on board the PROBA-2 satellite on November 2, 2009. With four channels new technology components were tested covering the wavelength intervals of $115-125 \mathrm{~nm}, 200-220 \mathrm{~nm}, 17-50 \mathrm{~nm}$, and 1$20 \mathrm{~nm}$. The optical components are filters and special diodes (Hochedez et al., 2006). The platform was launched in a Sun low Earth orbit (altitude of $725 \mathrm{~km}$ ) with an inclination of $98.28^{\circ}$.

Calibration is performed on ground, by onboard calibration light sources (light-emitting diodes), and through inter-calibration with other space missions.

\subsubsection{CORONAS-PHOTON/PHOKA}

The satellite CORONAS-PHOTON (Kotov et al., 2011; Pflug et al., 1996) was launched in January 30, 2009. The EUV PHOKA instrument aboard recorded radiation in the spectral windows of 0.5-7 nm, 0.5-11 nm, 27-37 nm, and 116-125 nm, respectively until January, 2010. The photodiodes of the instrument were calibrated before launch using a synchrotron radiation source.

\subsubsection{SDO/EVE}

Since the launch of the SDO satellite on February 11, 2010 the EUV Variability Experiment (EVE) measures the EUV in the 6-106 nm range with $0.1 \mathrm{~nm}$ spectral resolution with 10-sec cadence, and the 0.1-7 nm band with 0.25 -sec cadence.

The EUV SpectroPhotometer (ESP, Didkovsky et al., 2012) channel of the EVE/SDO instrument has provided photometrically accurate $(<\sim 10 \%$ absolute uncertainty), 0.25-sec cadence irradiance data in four EUV/soft X-ray bands virtually without interruption since the end of the commissioning phase in April 2010. The ESP is an advanced version of the SOHO Solar EUV Monitor (SEM) that is based on the same transmission grating, thin film filter, and silicon photodiode technologies, but has additional features for in-flight calibration/degradation monitoring, and measures in a larger number of spectral bands, including 0.1-7 nm (zeroth-order quad diode Channels 4 through 7), 16.6-21.5 nm (Channel 8), 22.3-28.8 nm (Channel 2), and 26.2-33.8 nm (Channel 9).The Multiple EUV Grating Spectrographs (MEGS) measures the 6-106 nm spectral irradiance at a resolution of $0.1 \mathrm{~nm}$ and a time cadence of 10 -seconds. The MEGS channels use concave reflection gratings, thin free-standing foil filters, and back-illuminated CCDs (Woods et al., 2013). 
SDO is in a geosynchronous orbit and provides 247 observations of the Sun except for two short eclipse periods per year. However, following a power supply anomaly for the Multiple EUV Grating Spectrographs A (MEGS-A) channel, the high resolution spectra have not been available for wavelengths shorter than about $32 \mathrm{~nm}$ since May 26, 2014.

Calibration is performed on the ground, by inter- comparison with similar optical components of different exposure history to solar radiation, by inter-calibration with calibration rocket flights once per year, and with inter-calibration with other space missions such as SolACES and TIMED/SEE.(*)

\subsubsection{Future EUV SSI Observations}

As mentioned in Section 3.1.3, there is an on-going plan to monitor the SXR and EUV in four spectral bands with the GOES-R series of satellites through about 2030. The GOES XRS and EUVS measurements are only in a few bands. Understanding the SXR spectra it has been challenging performing measurements with just broad-band photometers in the SXR range (0.1-10 nm). This issue will be addressed in the near future with the launch of the NASA Miniature X-ray Solar Spectrometer (MinXSS) CubeSat in late 2015. The MinXSS will provide SXR spectral measurements using the Amptek X123-SDD sensor. - As Figure 1 illustrates, there is likely to be a future EUV Data Hole without full spectral coverage, much akin to the lack of solar EUV measurements in the 1980s and 1990s. Therefore, improving models of the solar EUV irradiance using these current EUV spectral observations is becoming increasingly more important as these satellites age.

\subsection{Absolute calibration in the EUV spectral range}

In the EUV spectral range, pre-flight on-ground calibration traceable to synchrotron radiation based standards can ensure the desired accuracy. However, in this spectral range the optics and detectors steadily suffer from loss of the initial calibration due to degradation. Degradation of each optical component is caused by diversity and unique diverse physical processes so that it is not possible to model the resulting efficiency changes - it must be measured (Schmidtke, 2015). The more components involved, the more difficult it is to determine the efficiency of the instrument. For example, each pixel of array detectors has to be considered a single optical component. Moreover, real components show non-uniform efficiency patterns. If the actual efficiency of an instrument is to be determined, the same partial area of each component interacting with the EUV photon fluxes must be taken into account for each spectral interval of interest. In this arrangement the efficiency curve with wavelength is fairly smooth.

Figure 2 demonstrates the difficult task of deriving reliable efficiency data for the San Marco 5 Airglow Solar Spectrometer Instrument (ASSI) aboard the San Marco 5 satellite (Schmidtke et al., 1985). The 18 channels of four spectrometers consisted of an optical grating and a photo-electron multiplier such as a secondary electron multiplier or a channel electron multiplier, each. The figure shows the Channel 16 relative efficiency changing with time and with wavelength (Schmidtke et al., 1995). The data is determined by cross-calibration with a rocket experiment (Woods and Rottman, 1990), tracking degradation of detectors using an 
internal radioactive source, and analysis of overlapping spectral regions of different channels (Worden et al., 1996).

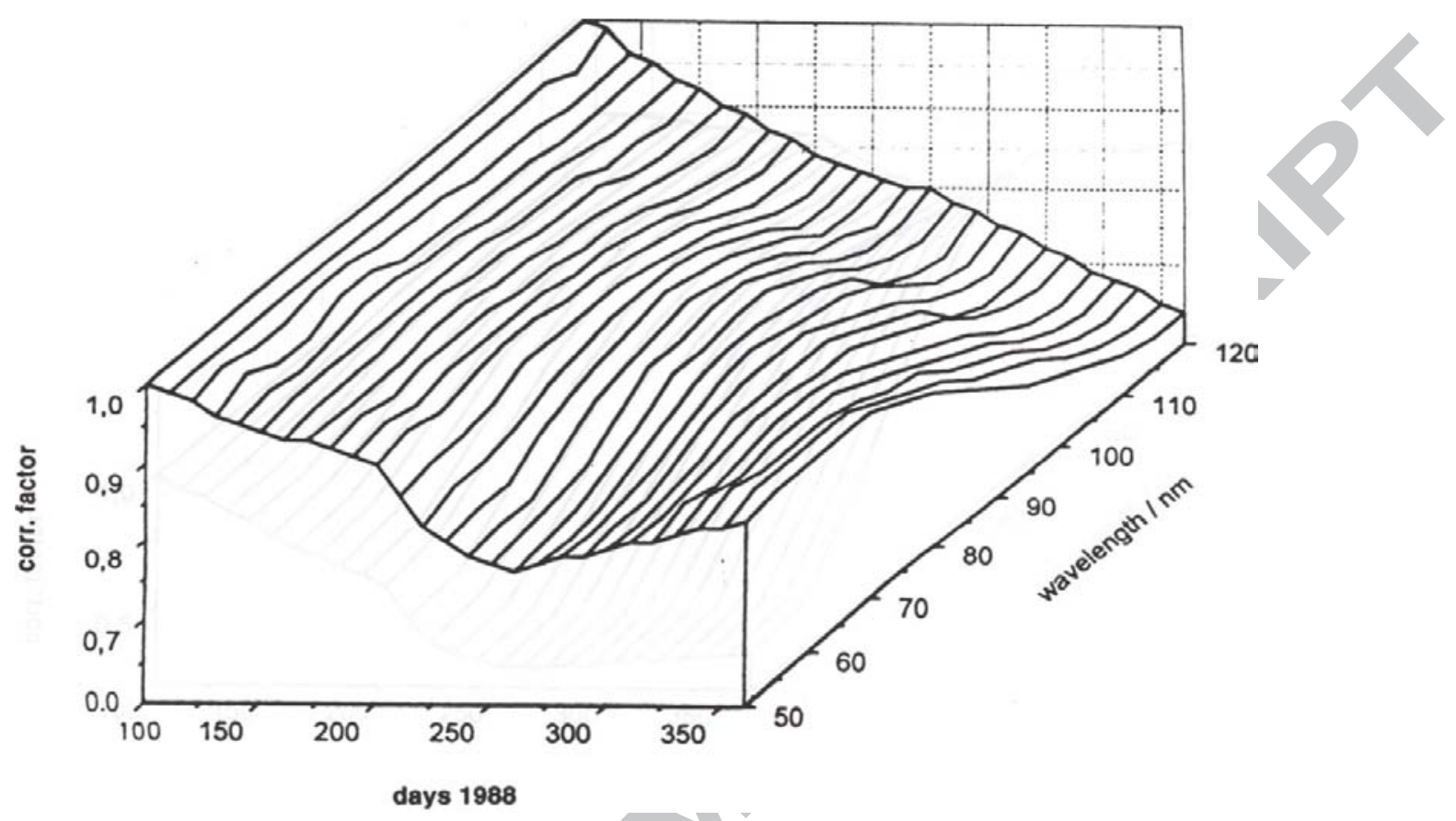

Figure 2: Relative efficiency changes of Channel 16 in the San Marco ASSI experiment withtime and wavelength (Courtesy by Worden and Woods, 1996).

Though it is difficult to separate efficiency changes from the optical grating and the electron multiplier as presented in Figure 2, the channeltrons as used in ASSI show linearity only under quite limited conditions such as low variability of count rates below 10exp(05) cps and accumulated count rates $<10 \exp (11)$. This is also true for the channeltrons of the SolACES experiment. Consequently, space experiments equipped with those detectors must be repeatedly re-calibrated during the mission.

The latter issue holds also for silicon photodiodes coated with thin-film metallic and/or for interference filters. For example, silicon photodiodes coated with aluminum-carbon (AlC) layers are subject to twofold efficiency changes, of the AlC filter and of the silicon photodiode, respectively. The exposure of the AlC filter to the full solar spectrum with photon energies exceeding $100 \mathrm{eV}$, to protons, to neutral and ionized atomic oxygen, and to cosmic radiation is subject to detachment of the upper layers of the metallic film, thus increasing the filter transmission, hence increasing also the recorded current. It is suspected that the deviation of the SEE data from the SEM, SolACES, and EVE recordings could be explained by this effect (see Figures 9 and 10 in Section 5.1). This view is supported by the deviation of the SEE data continuously increasing with time of exposure. In addition, the photodiodes themselves are subject to irradiation induced efficiency changes, too, which occur even under controlled irradiation conditions for traceable calibrated detectors (Gottwald et al., 2011; Scholze et al., 2010). 
In order to save valuable resources for estimating the aging of EUV instruments on the ground as well as in space the primary objective of future work must include establishing instrumentation and methodologies providing real-time EUV SSI data sets to be used as a common standard for cross-calibration purposes. The state of development realizing this objective is ready to be started.

One possible way to realize this goal is based on the work by Samson (1967): "The only absolute primary detector available in the vacuum ultraviolet (VUV) spectral region makes use of the ionization effect of radiant energy. In one class of detectors (ion chambers with rare gases) one photon absorbed produces one ion-electron pair."

There are two types of ionization chambers, high-field and low-field chambers (Samson and Haddad, 1974). The measuring principle with re-calibration capability in space was first published in 1976 (Schmidtke, 1976a). EUV radiation is entering ionization chambers via monochromators or narrow-band filters as realized in the SolACES experiment. In order to mitigate secondary ion-electron production for higher EUV photons in SolACES a low-field chamber is applied as a double chamber with two ampere meters. A third current from a silicon detector at the end of the chamber is generated by the photons passing the two chamber segments (Figure 3). The latter current is not impeded by secondary ionization effects. For each of the IC measurements the chambers are filled up with gas. The actual filter transmission is repeatedly determined in space (Schmidtke et al. 2006b; Schmidtke et al., 2014).

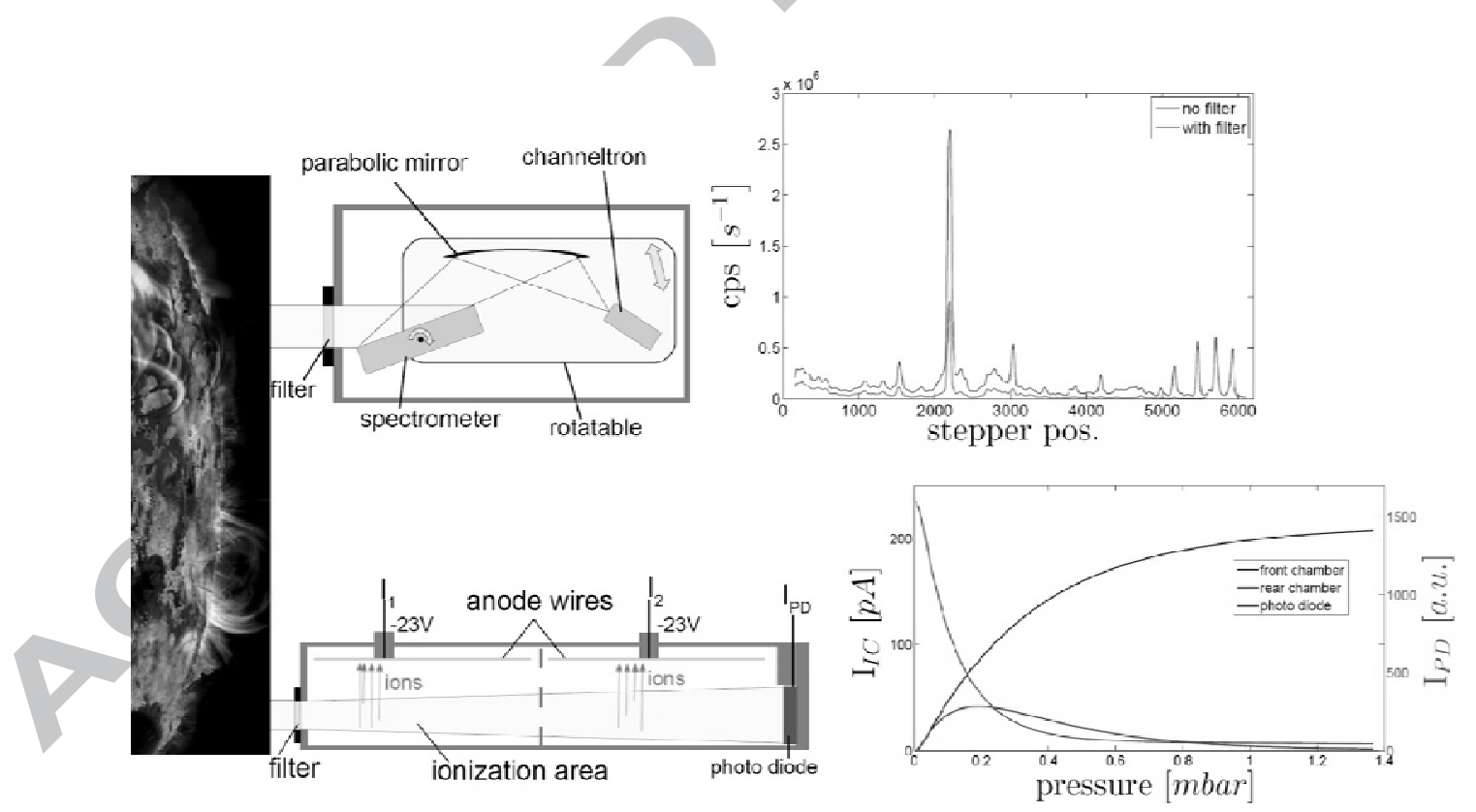

Figure 3: Schematics of SolACES: Solar radiation is entering either the spectrometers with or without filters on top of the aperture producing count rates (upper part) or the ionization chambers producing three currents (lower part). 


\subsection{Recent and on-going UV measurements}

The history of UV measurements at wavelengths longer than $200 \mathrm{~nm}$ from orbit started with NIMBUS 7 in 1978. Given the series of missions with appropriate overlapping periods, the continuity of measurements has been ensured. We shall present only the three current missions operating after 2014. The prior solar EUV measurements include the following.

- The Solar Backscatter UltraViolet (SBUV, Cebula et al., 1992), onboard the National Oceanic and Atmospheric Administration (NOAA) spacecrafts series,

- The Solar Ultraviolet Spectral Irradiance Monitor (SUSIM, Brueckner et al., 1993), and the SOLar STEllar Irradiance Comparison Experiment (SOLSTICE, Rottman et $a l ., 1993)$ for the UV, onboard the Upper Atmosphere Research Satellite (UARS),

- The SCanning Imaging Absorption spectrometer for Atmospheric CHartograpY (SCIAMACHY, Bovennsmann et al., 1999) onboard the ENVISAT satellite,

- The SOLar-STellar Irradiance Comparison Experiment (SOLSTICE) and Spectral Irradiance Monitor (SIM, Harder et al., 2010) on the Solar Radiation and Climate Experiment (SORCE, Rottman, 2005).

- The SOLar SPECtrum (SOLSPEC, Thuillier et al., 2004, 2009) on board the International Space Station.

- PREcision MOnitoring of Solar variability, (PREMOS, Schmutz et al., 2009) on board PICARD satellite.

$(*)$

The UV spectrometers have frequently experienced loss of their spectral responsivity caused by contaminants surface deposition on the optical components generated by the spacecraft/platform environment or instruments outgassing and space particles. Organic contaminants being exposed to EUV are pyrolyzed. This process can produce a permanent carbon layer on the optical surface. Carbon has a strong capability of absorption in EUV and UV that causes absorption of the solar photons. Furthermore, assuming no contamination, the energetic EUV radiation alters the optical components characteristics (transmission, reflectivity, detectors efficiency) leading to significant changes in the instrument efficiency. Furthermore, water vapor, which has a very slow outgassing rate from the instruments in space, interacts with the optical surfaces and forms oxidation layers that also absorb the solar EUV/UV radiation. Depending of the mechanical configuration and the thermal environment, ice layers can be deposited on the optical surfaces. Various solutions are employed to monitor the aging in space.

\subsubsection{SOLAR/SOLSPEC}

SOLSPEC consists of three spectrometers, dedicated to the measurements of the UV, visible and infrared (IR) solar irradiance. They are described in detail by Thuillier et al. (2009). SOLSPEC also includes a set of lamps to monitor the instrument degradation, the wavelength scale and spectrometer slit function. Each spectrometer consists of a double monochromator using holographic gratings manufactured by Jobin-Yvon.

The six gratings are placed on a one-piece housing rotating thanks to a stepper motor having an angular position precision corresponding to a spectral sampling of $0.01 \mathrm{~nm}$ precision. Second order and attenuator filters are placed on the optical path as a function of the grating 
step number (i.e. wavelength). At the entrance of each spectrometer, a quartz diffuser allows us to reduce the signal dependence of the sunline of sight position with respect to the instrument optical axis.

In order to prevent the aging of the three optical entrances generated by solar EUV photons, mobile quartz plates, and a hole are set on a wheel. Two plates are used per spectrometer. One is routinely used. The second one is a spare unit used once a month, which allows us to measure the ratio of their transmission. The absolute transmission is also measured by removing the plates from the optical entrance. This operation is made every six months.

Two deuterium lamps for the UV spectrometer and two tungsten ribbon lamps for the visible and infrared spectrometers allow in flight monitoring of the spectrometers responsivity. A decrease of the solar as well as the deuterium lamps signals appeared having an unexpected high rate of change (about $60 \%$ per year). In April, 2009 the lamp power supply failed. The aging significantly decreased to reach about $10 \%$ per year afterward. This change of rate associated with no further use of the deuterium lamp has been interpreted as due to the outgassing of a material used to maintain the lamp in its support, likely some glue given the high temperature reached by the lamp in function (about $170^{\circ} \mathrm{C}$ ). A hollow cathode lamp provides lines used to measure in flight, the slit function, and the spectrometers dispersion law.

The auto-calibrating instruments SOLSPEC and SolACES are mounted on a solar pointing system attached to the COLUMBUS ESA module on the International Space Station (ISS). However, solar viewing cannot be achieved every day due to the ISS orientation preventing to observe a complete solar rotation. The mean duration period of the Solar Visibility Window (SVW) is about two weeks. At solstice, the duration of the eclipse reduces to about 6 days. On demand 'bridging' maneuvers of the ISS changing the attitude during up to six days two of the SVWs were connected such that full solar rotation periods were observed.

\subsubsection{SORCE/SIM}

The NASA SOlar Radiation Climate Experiment (SORCE) has been making daily measurements of the total solar irradiance (TSI) and solar spectral irradiance (SSI) since its launch in 2003 and is expected to continue measurements until 2018 allowing for overlap with the NOAA/NASA Total and Spectral Irradiance Sensor (TSIS) that will be launched to the ISS in late 2017. Aboard SORCE are two SSI UV spectrometers: the Solar Irradiance Monitor (SIM) and SOLar and STellar Irradiance Comparison Experiment (SOLSTICE).

The SIM is a dual Féry prism spectrometer that employs five detectors per spectrometer channel to cover the wavelength range from 200 to $2400 \mathrm{~nm}$. Two identical, mirror-image, channels are used for redundancy and in-flight measurement of prism degradation. The primary detector for this instrument is an Electrical Substitution Radiometer (ESR) designed to measure power levels 1000 times smaller than other radiometers used to measure TSI. The four complementary focal plane photodiodes are used in a fast-scan mode to acquire the solar spectrum daily, and the ESR calibrates their radiant sensitivity about once a week. Wavelength control is precisely achieved by using a closed loop servo system that employs a linear charge coupled device (CCD) in the focal plane to achieve 0.67 arc second control of 
the prism rotation angle. The SIM / SORCE instrument and calibrations are described in detail by Harder et al. (2005a) and Harder et al. (2005b).

Calibration is performed on ground, by inter-calibration with redundant channel about once per month, and with inter-calibration with other space measurements such as by SOLSTICE/SORCE and SOLSPEC/ISS.

\subsubsection{PICARD/PREMOS}

The PREMOS experiment comprises several instruments aboard the PICARD solar mission, and aims at measuring spectral and total solar irradiance using filter sunphotometers and absolute radiometers respectively. PREMOS observations extend from July 27, 2010 to April, 2014. The photometric channels are centered at 210, 215, 266, 535, 607, and at $782 \mathrm{~nm}$. The data reduction of the PREMOS measurements will be addressed in a forthcoming paper. We have available final data sets for the UV channels for which the degradation has been successfully corrected with back-up channels. Those measurements at 210 and $266 \mathrm{~nm}$ are particularly suited for the study of rotational variability as showed by Cessateur et al (2015).

\subsubsection{SORCE/SOLSTICE}

The SOLSTICE / SORCE measurements of the solar UV irradiance are from $115 \mathrm{~nm}$ to 320 $\mathrm{nm}$ with a spectral resolution of $0.1 \mathrm{~nm}$ and with an accuracy better than $5 \%$ and long-term stability of better than $0.5 \%$ per year. This instrument continues on UV measurements of the SOLSTICE / UARS instrument that has similar technology but was over the spectral range of $120 \mathrm{~nm}$ to $410 \mathrm{~nm}$. The SOLSTICE / SORCE has a pair of identical scanning grating spectrometers that can measure both solar and stellar irradiance. Each spectrometer has entrance slit, flat mirrors for folding the optical path, diffraction grating, concave focusing mirror, exit slit, exit filters for reducing light for the brighter wavelengths, and photomultiplier tube (PMT) detectors. Both UARS and SORCE versions of SOLSTICE are designed to use bright, stable, main-sequence $\mathrm{O}$ and $\mathrm{B}$ type stars for their in-flight calibration. In order to measure six orders of magnitude difference between solar and stellar irradiance, they have interchangeable entrance and exit slits, with the larger slits used for the stellar observations, and the ability to integrate longer for the stellar observations. The SORCE/SIM instrument and calibrations are described in detail by McClintock et al. (2005a) and McClintock et al. (2005b).

Calibration is performed on ground, by inter-calibration with its redundant channel about once per week, and with inter-calibration with other space measurements such as by SORCE/SIM and UARS/SUSIM.

\subsubsection{AURA/OMI}

The Ozone Monitoring Instrument (OMI) aboard the NASA AURA mission observes Earth's ozone as it primary measurements but also observes the solar irradiance for monitoring its instrument degradation trend. The OMI solar measurements cover the range of $265 \mathrm{~nm}$ to 500 $\mathrm{nm}$ and have been made about once per week since July, 2004 (Marchenko and DeLand, 
2014). The OMI solar measurements also include the $\mathrm{Mg} \mathrm{II} \mathrm{h/k}$ and $\mathrm{Ca}$ II $\mathrm{K}$ indices that have been valuable as proxies of chromospheric variability (DeLand and Marchenko, 2013).

\subsubsection{Future UV SSI Measurements}

The NOAA/NASA TSIS program is expected to continue the solar UV SSI measurements in the range of $200 \mathrm{~nm}$ to $2400 \mathrm{~nm}$ for the foreseeable future. The TSIS- 1 instruments, both for TSI and SSI by a second generation of SIM, have been built and calibrated and await launch to the ISS in late 2017. The TSIS-2 mission is currently being defined but is likely to continue with third generation TSI and SSI instruments from the SORCE and TSIS-1 heritage.

\subsection{Absolute calibration in the UV spectral range}

The pre-flight calibrations of the UV SSI instruments are based primarily on radiometric source standards, such as tungsten and deuterium lamps (secondary standards) and synchrotron radiation (primary standard). For example, the SUSIM and SOLSTICE instruments were calibrated before launch using the Synchrotron Ultraviolet Radiation Facility (SURF) at the National Institute for Standards and Technology (NIST) in Gaithersburg, MD. The instrument papers by Thuillier al. (2009), Harder et al. (2005b), and McClintock et al. (2005b) provides more details about pre-flight calibrations. The absolute accuracy of such calibrations is typically $1-10 \%$, wavelength dependent and source dependent. Besides source-based radiometric methods, the use of cryogenic radiometers as primary detector standards (either with laboratory-based or synchrotron radiation sources) is possible. Only recently cryogenic reference detectors have been used for SSI instrument calibrations, such as for TSIS-1/SIM, to improve upon the SSI instrument accuracy to better than $0.1 \%$.

\section{SSI and related modeling}

\subsection{EUV/UV SSI indices}

Several indices (indicator of a wavelength band activity level) and proxies (substitutes for other wavelength bands) of solar activity have been developed to compensate for the paucity of direct UV/EUV observations. The Covington index $F_{10.7}$ is probably the one of the widely used proxies because it mimics the SSI in the EUV band relatively well. Observed on ground since 1947, it is derived from solar radio emission at $10.7 \mathrm{~cm}$ wavelength hence, it is available without using expensive space technology. It provides the longest legacy time series for modeling solar EUV emissions.

However, the correlation of the $\mathrm{F}_{10.7}$ EUV proxy with other spectral bands is less satisfactory. This has stimulated the search for other indices and proxies such as daily quantities for the JB2008 satellite drag model (Tobiska et al., 2008). In addition to $F_{10.7}$ three other indices have been developed to better represent the combination of solar source temperature region and Earth-atmosphere unit optical depth $(\tau)$ combination. These include:

S10 (EUV 26-36 nm: chromosphere emission with $\tau>180 \mathrm{~km}$ ), 
M10 (FUV $160 \mathrm{~nm}$ : photosphere SRC emission with $\tau \approx 100 \mathrm{~km}$ ), and

Y10 (0.1-0.8 nm plus $121 \mathrm{~nm}$ : coronal and transition region emission with $\tau \approx 90 \mathrm{~km}$ ).

The JB2008 indices were developed using concepts originally proposed in 1976. EUV indices $E V_{\mathrm{x}}$ and $\mathrm{EUV}_{\mathrm{yz}}-\mathrm{EUV}-\mathrm{SSI}$ for solar-terrestrial relations (with $\mathrm{x}$ - wavelength and $\mathrm{yz}$ interval of wavelengths) were first proposed by Schmidtke (1976b). This idea is also pursued with the TIGER/COSPAR initiative (Schmidtke et al., 2006a). Based on the past and planned work on SSI data the indices in use such as F10, S10, M10, and Y10 show to substantially improve neutral thermospheric mass density modeling and are adapted to other applications such as ionospheric modeling. Related applications for physical modeling issues in solarterrestrial relations are considered such as:

EUV-TEC - models of the ionosphere taking into account energy loss by ionization,

EUV-THERM - models of the thermosphere taking into account EUV albedo radiation,

EUV-F $F_{10.7}$ - EUV-TEC normalized to the $F_{10.7}$ index for replacing $F_{10.7}$ in models used,

EUV-CHARGING - to be used computing spacecraft dis-charging by solar EUV fluxes. The solar centimetric flux at other wavelengths can be a valuable proxy for the UV. The radio flux at $30 \mathrm{~cm}$ (or F30 index), for example, is a good proxy for the EUV-NUV band, and as such has been recommended for satellite drag modeling (Dudok de Wit et al., 2014).

\subsection{EUV/UV SSI modeling for solar-terrestrial relations}

Measuring the SSI in the EUV/UV is a highly challenging task, still. For practical use different versions of SSI data are required depending on their application. For example, the requirements for the modeled solar SSI data to be used in ionospheric models differ substantially with respect to wavelength interval, accuracy, and spectral resolution from those used in models of the upper mesosphere. Having in mind the challenges of accurate EUV/UV monitoring, the lack of real-time data, the often diverging results from different instruments, and temporary data gaps, modeling of solar EUV/UV SSI data is mandatory. Thanks to the already existing modeled SSI data sets significant progress has been made in the field of solar-terrestrial relations.

The key aim of SSI modeling is to provide the temporal variability for the full or parts of the solar spectrum typically and for times when no EUV/UV solar spectral irradiance is available. Such models follow either a theoretical or empirical approach or are a combination of both. Depending on the approach different proxies are employed. Periodical variations are of importance, i.e. the 27-day rotation and the 11-year solar cycle including, and the long-term variations from the Maunder minimum to the present.

Depending on the user community the requirements on the SSI data sets are different. For example, modeling climate and atmospheric physical processes require different temporal resolution of the data sets applied. While climate modeling covers epochs over solar cycle periods with increasing interest to its absolute value, atmospheric physics require both 
variability and absolute value as solar models are used in atmospheric models for calculation of the atmospheric species through different photochemical reactions such as photodissociation, ionization, recombination, and others.
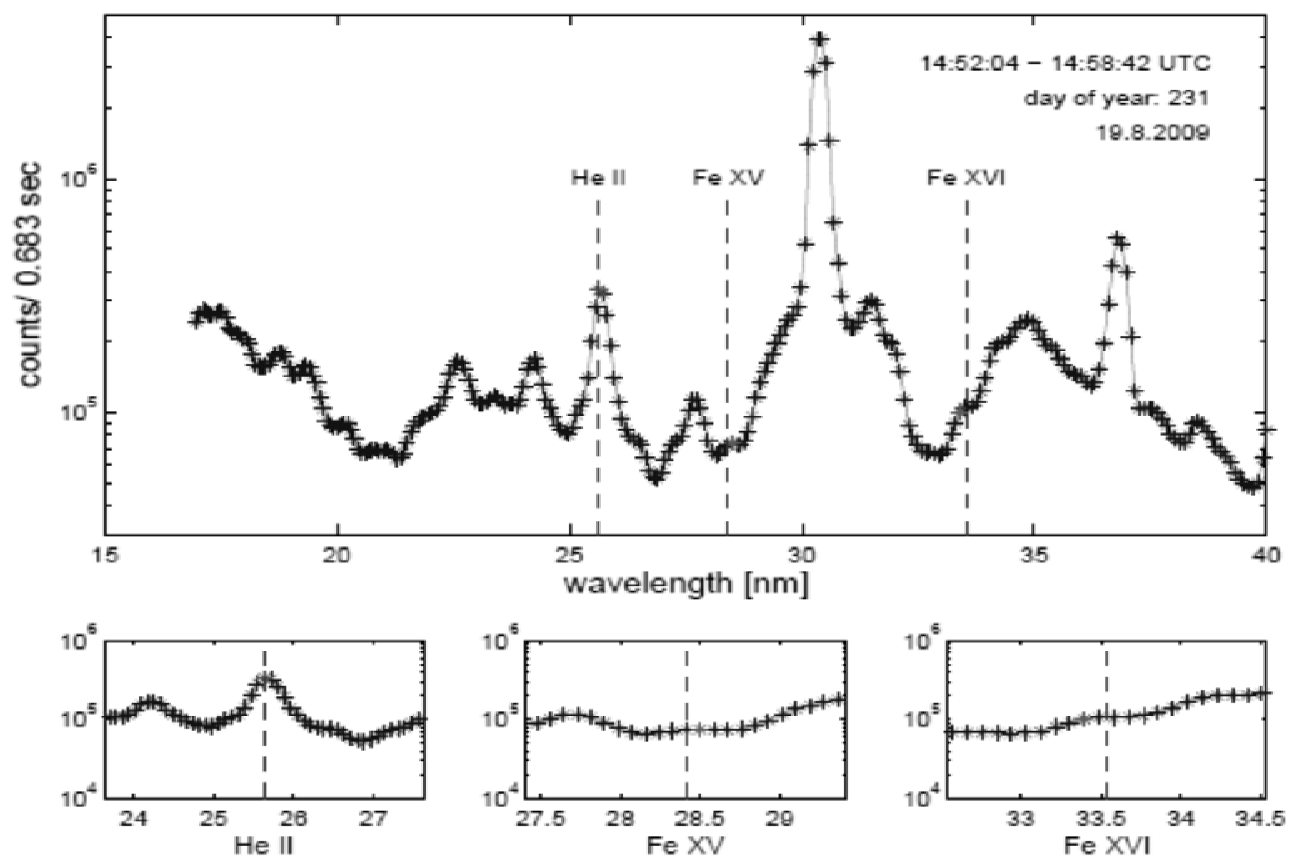

Figure 4a: Fe line emissions at solar minimum activity in 2009 in the absence of significant Sunspots.
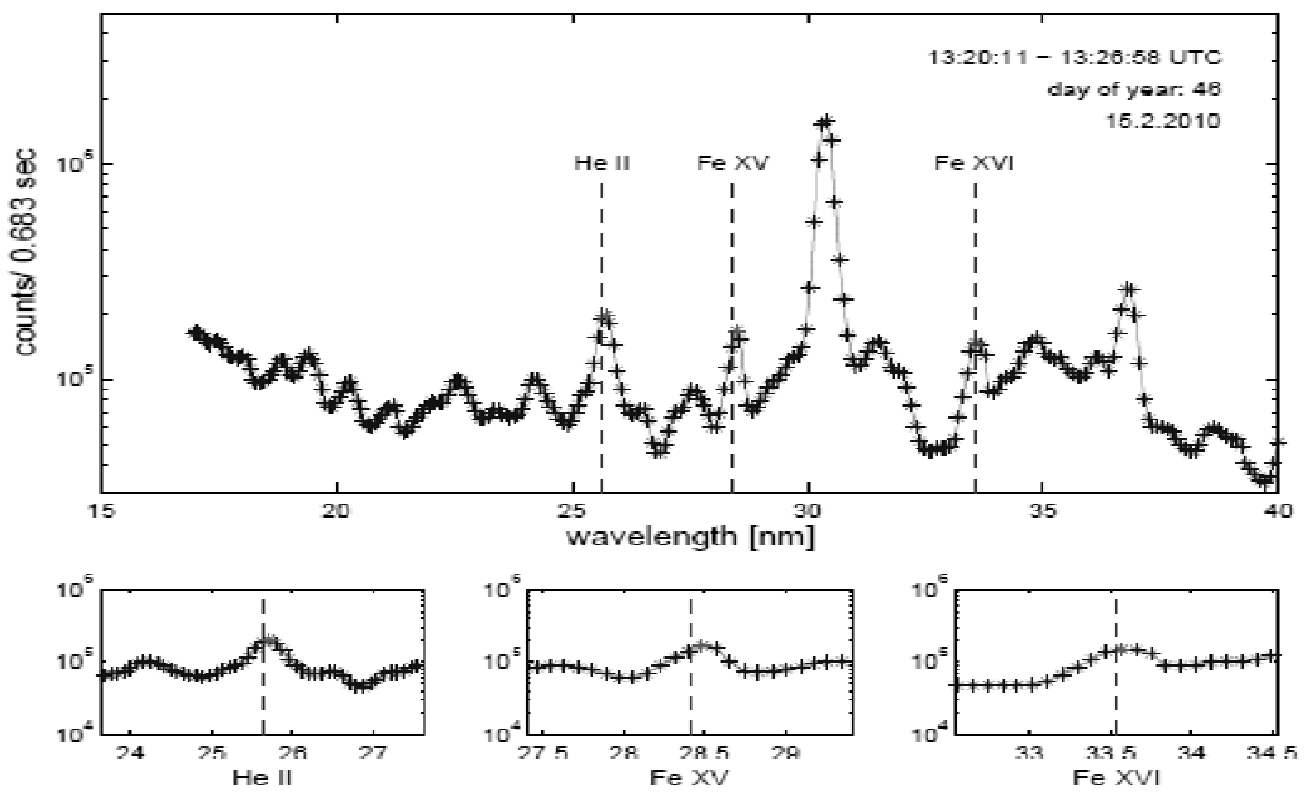

Figure 4b: Fe line emissions at solar minimum activity in 2009 in the presence of Sunspots. 
Based on a statistical analysis of the EUV data recorded aboard the aeronomy satellite Atmospheric Explorer-E Hinteregger (1977, 1981; Hinteregger et al., 1981) developed a reconstruction approach for the full spectral coverage by using two 'key' emissions, namely $\lambda_{\text {Ref }}=102.7 \mathrm{~nm}$ of H I and $\lambda_{\text {Ref }}=33.5 \mathrm{~nm}$ of Fe XVI. These parameters represent two classes of solar activity, namely medium and highly variable solar emissions, respectively. Assigning a class assignment to each wavelength, ranging from $1.86 \mathrm{~nm}$ to $209 \mathrm{~nm}$, a variability index, and the ratio of the intensities of the two 'key' emissions derived for a given day the corresponding intensity can be computed. As Figures 4, panel a,b demonstrate this procedure seems not being applicable for the 'key' emission Fe XVI during solar minimum conditions. While the increase of the Fe XVI intensity from August 19, 2009 (Fig. 4a) to February 15, 2010 (Fig. 4b) is about a factor of 4, the great number of the Fe VIII to Fe XII emissions in the important spectral range from $16 \mathrm{~nm}$ to $28 \mathrm{~nm}$ is increased by less than a factor of two. The question comes up: Does this procedure with variability indices that are linearly related to the two 'key' emissions represent e.g. the great number of Fe VIII to Fe XII emissions in the important spectral range from $16 \mathrm{~nm}$ to $28 \mathrm{~nm}$ from the absence to the appearance of Sunspots during a solar cycle from solar minimum to solar maximum conditions? This is not likely because the Fe XVI emission is generated in the hot regions of the corona while the $\mathrm{Fe}$ VIII/XII emissions are primarily originated in the transition region. Similarly, the local excitation of the hydrogen emission at $102.7 \mathrm{~nm}$ differs from the regions where helium, neon, oxygen and other elements are emitting.

Concluding from SolACES observations a set of minimum four 'key' emissions could improve the representation of full EUV spectra emerging from the solar lower (hydrogen $\mathrm{H} \mathrm{I}$ at $102.7 \mathrm{~nm}$ ) and upper chromosphere (carbon C III at $97.7 \mathrm{~nm}$ ), each formed in the transition region (iron emissions between 18-22 nm), and the corona (Fe XV at $28.4 \mathrm{~nm}$ ). These emissions are still to be considered arbitrary. It is necessary to investigate, if other lines would lead to more accurate results. This issue is further discussed in Section 6.7.

\subsection{EUV/UV SSI modeling for solar physics}

A different approach in EUV/UV SSI modeling is required for solar physics as compared to applications in solar terrestrial relations (see Section 2).

4.3.1 Modeling of the 27-day solar rotation SSI variability

Using the measurements obtained during about 45 days, the SSI variation caused by the solar rotation has been observed by the instruments onboard the ISS for the first time in December, 2012. Data from other spacecrafts and modeling are also used:

- PREMOS (Schmutz et al., 2009) has been operated onboard the PICARD spacecraft,

- Extreme ultraviolet Variability Experiment (EVE) onboard Solar Dynamics Observatory (SDO, Woods et al., 2010), 
- Solar EUV Experiment (SEE) onboard Thermosphere Ionosphere Mesosphere Energetics and Dynamics (TIMED, Woods et al., 2005),

- Solar Radiation and Climate Experiment (SORCE, Rottman, 2005) instruments.

SORCE monitors the solar spectral irradiance using three instruments the SOLar STellar Irradiance Comparison Experiment (SOLSTICE, 115-320 nm, Rottman, 2000), the Solar Irradiance Monitor (SIM, 200-2400 nm, Harder et al., 2005a; 2005b), and the XUV Photometer System (XPS, 0.1-34 nm and Ly $\alpha$ ).

PICARD/PREMOS uses absolute radiometers and filter sunphotometers to measure the total and solar spectral irradiance, respectively. Several spectral domains are observed, among them, we have used the $215 \mathrm{~nm}$ range having a width of $10 \mathrm{~nm}$.

Two EUV models are used :

- The SOLar MODelling code (SOLMOD, Haberreiter, 2011; Haberreiter et al., 2014) utilizes physical descriptions of the solar atmosphere in quiet and active regions to estimate their contributions to EUV irradiance changes,

- NRLSSI (Lean et al., 2011) uses wavelength dependent statistical relationships between the solar EUV irradiance measured by TIMED/SEE from 2002 to 2014 with solar activity proxies.

Five current UV models are discussed below. Let us stress that they highly differ in their level of sophistication. Some are mere linear combinations of solar proxies, whereas others involved solar spectral modelling:

- COSIR (Cessateur et al., 2015),

- MOCASSIM (Bolduc et al., 2012),

- MGNM (Thuillier et al., 2012),COSI (Haberreiter et al., 2008; Shapiro et al., 2010),

- NRLSSI (Lean, 2000),

- SATIRE (Krivova, 2010).

A detailed description of these models and the comparison of the respective predictions is given in Thuillier et al. (2014b), and briefly presented below. 


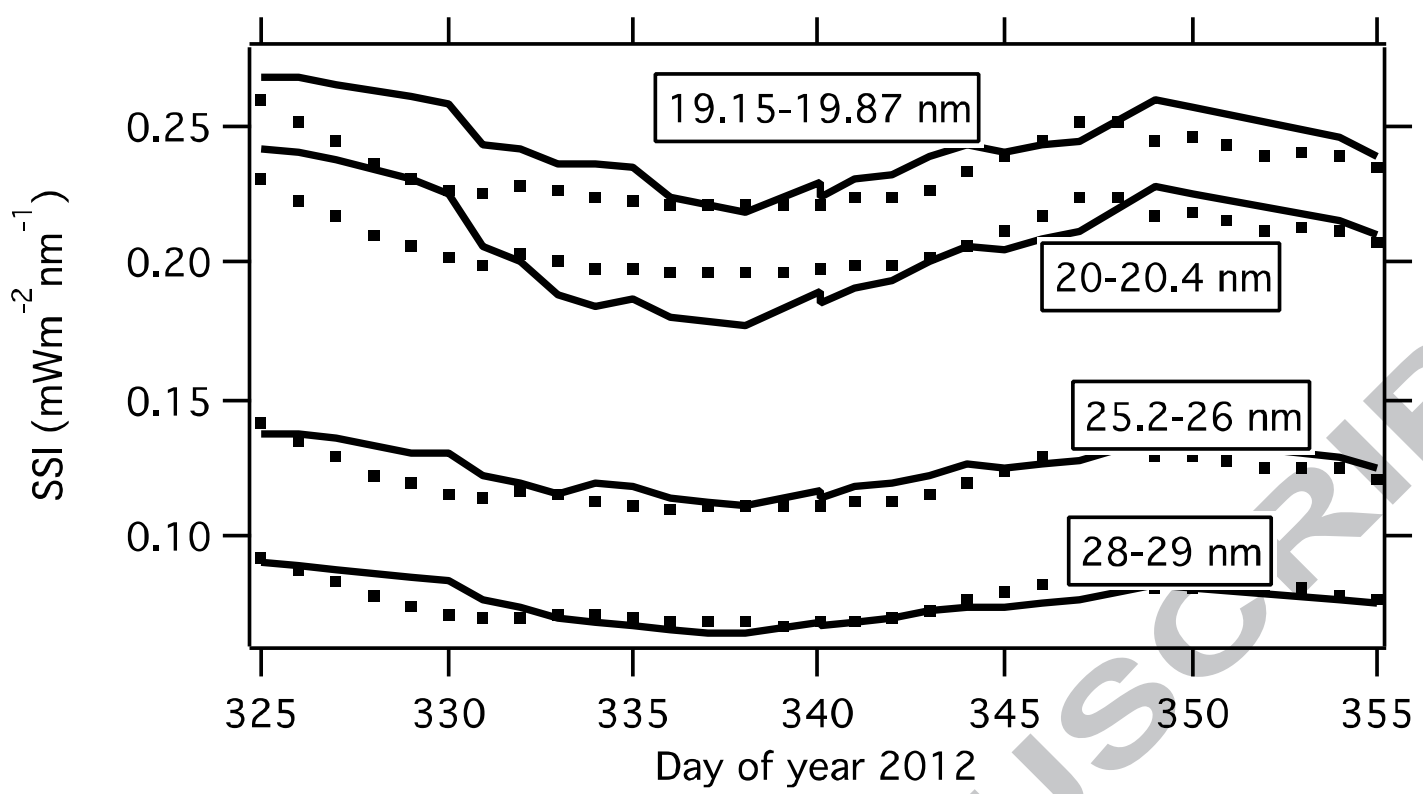

Figure 5a: Comparison between SSI variations (solid lines) measured by SolACES and modeling by SOLMOD (dotted lines) during the solar rotation of December, 2012.

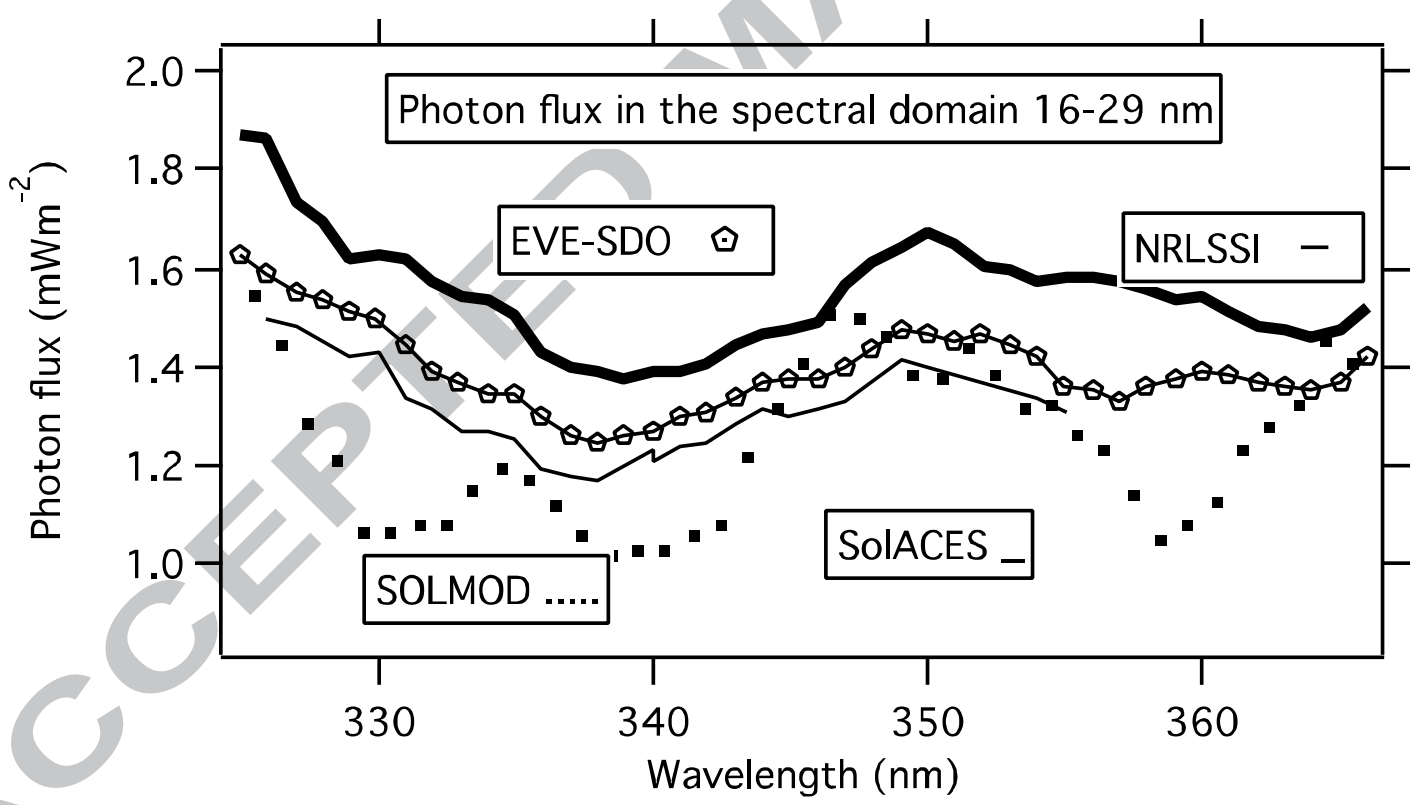

Figure 5b: Comparison of SolACES, EVE with modeling using NRLSSI and SOLMOD in the domain 16-29 $\mathrm{nm}$.

Figure 5a shows the EUV reconstruction for the five spectral intervals 19.15-19.8 nm, 20-20.4 $\mathrm{nm}, 25.2-26 \mathrm{~nm}, 28-29 \mathrm{~nm}$, measured with SolACES and modeled with SOLMOD. The phase of the solar rotation is correctly modelled, however, the absolute irradiance has been adjusted to SolACES, with a wavelength-dependent correction being applied. Currently, a new EUV 
reconstruction zsing an updated atomic dataset in SOLMOD is in preparation. The maximum to minimum irradiance ratio predicted by SOLMOD is, as expected, wavelength dependent and varies from 1.17 to 1.52 . The SolACES measurements indicate a variability ranging from 1.19 to 1.27. A similar comparison has been carried out with NRLSSI. The phase of solar variability is correctly modelled, and, again, as expected, the variability is wavelength dependent ranging from 1.22 to 1.30. As NRLSSI data are provided in $1 \mathrm{~nm}$ bin, and given the number of emission lines compiled in bin intervals, a comparison of predicted with measured value in absolute scale is not possible. This is why a large domain has been chosen extending from 16 to $29 \mathrm{~nm}$. The two EUV reconstructions along with the SolACES and SDO-EVE measurements are displayed in Figure $5 \mathrm{~b}$.

The phases of the solar cycle as observed by SolACES and EVE are in agreement with NRLSSI, however to a lesser agreement with SOLMOD, for which a new reconstruction is in preparation. The absolute values of NRLSSI, which is based on based on the SEE data, are about $25 \%$ larger than the measurements. The difference is understood to be due to differences in the absolute calibrations of the SEE and EVE instruments. SOLMOD reconstruction has been shifted by $+0.1 \mathrm{~mW} / \mathrm{m}^{2}$, and shows a longer rotational variation than the observations as likely due to the uncorrected straylight in the AIA observation.

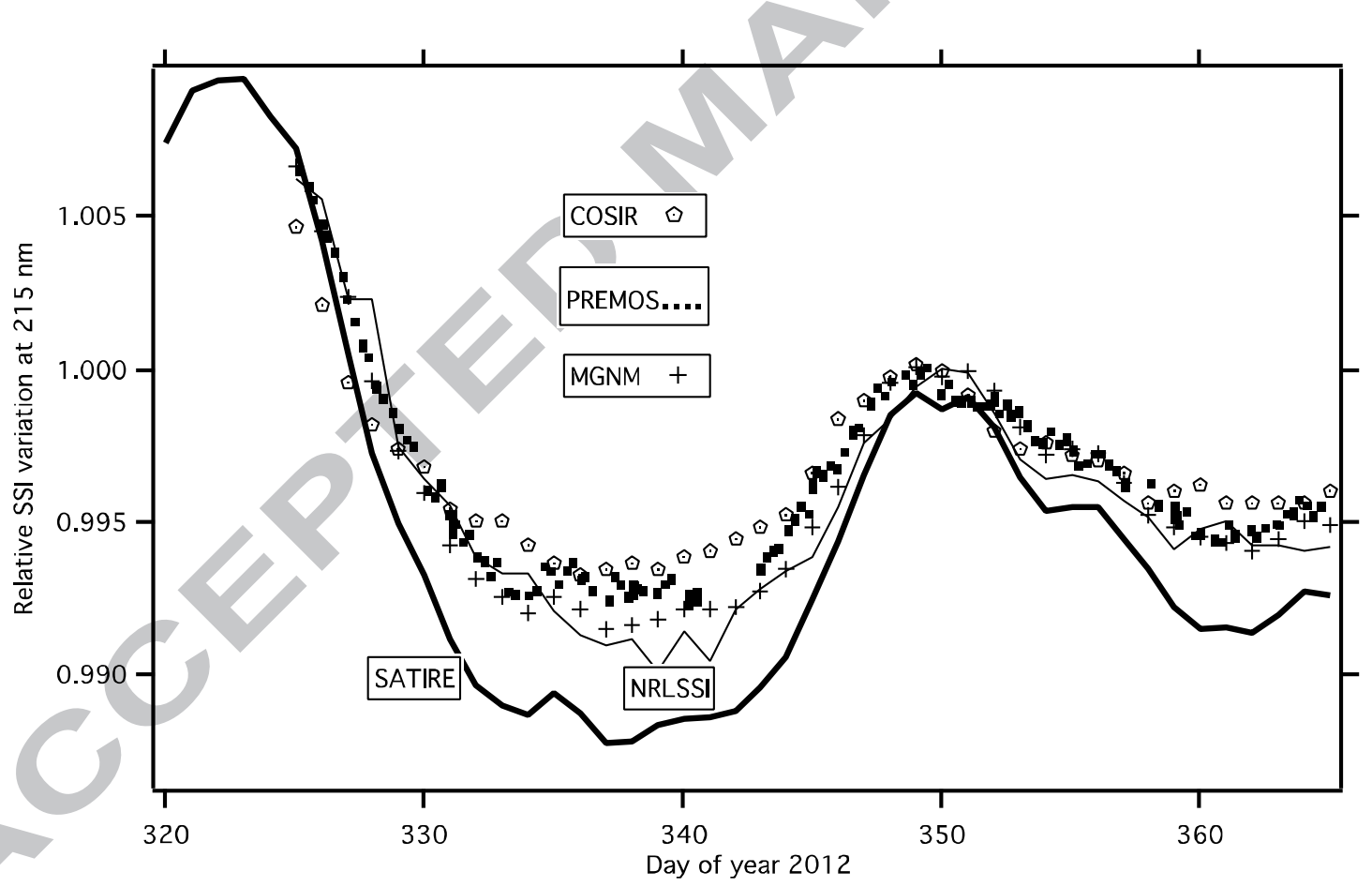

Figure 5c: PREMOS comparison at $215 \mathrm{~nm}$ with modeling.

At longer wavelength, fewer discrepancies are found between observations and reconstructions. The models reported above are used for reconstruction of the SSI at $215 \mathrm{~nm}$ variation generated by the December, 2012 solar rotation. The predictions are normalized to unity at the maximum SSI occurring at day 350 (Figure 5c). We first note that the phases by all models are in agreement with the measurements at $200 \mathrm{~nm}$ obtained with PREMOS as well 
as SOLSPEC (not shown on the figure). Simultaneously observed variability by PREMOS and SOLSTICES are 0.6 and 1\%, respectively. Models predict a variability ranging from 0.6 to $1.3 \%$, the latter being provided by SATIRE in agreement with Figure 5c. Maximum SSI extends from 33 to $37 \mathrm{mWm}^{-2} \mathrm{~nm}^{-1}$. A detailed analysis of the SSI predictions and their comparisons with measurements may be found in Thuillier et al. (2014a).

\subsubsection{Long term modeling}

Thuillier et al. (2013) have achieved a detailed analysis of the properties of five reconstructions as given above, however using COSI (Haberreiter et al., 2008; Shapiro et al., 2010) rather than COSIR as being better adapted to long term predictions Each model uses a spectrum either theoretical or measured, on which a variability is applied calculated from the various proxies used by each model. The specific methods of reconstructions used here are summarized in Thuillier et al. (2013).

The reconstructions differ mainly in terms of variability and absolute SSI as can be seen by comparing with measured SSI provided by SOLSPEC. They may also differ in term of spectrum. This is generally due to the spectrum used either measured or theoretical.

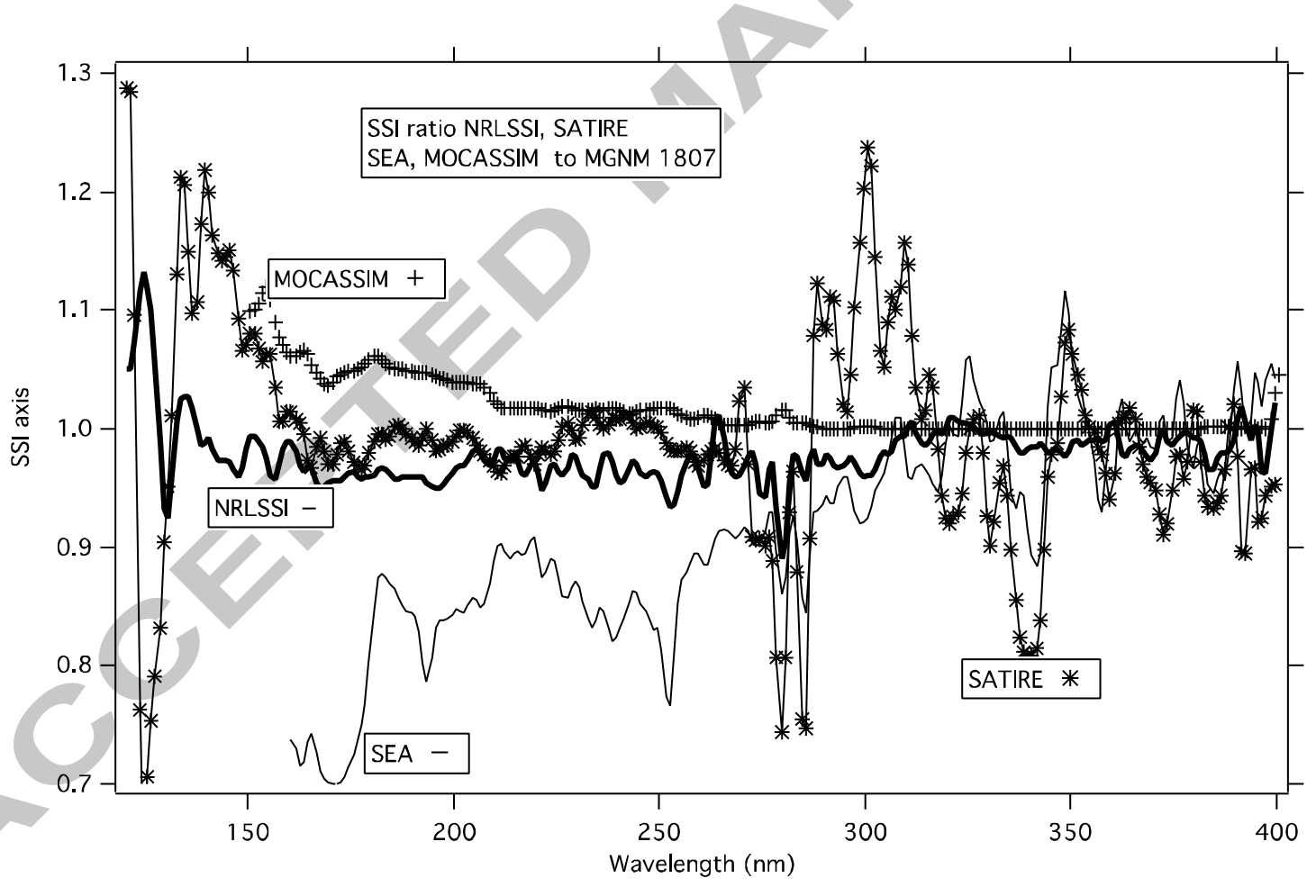




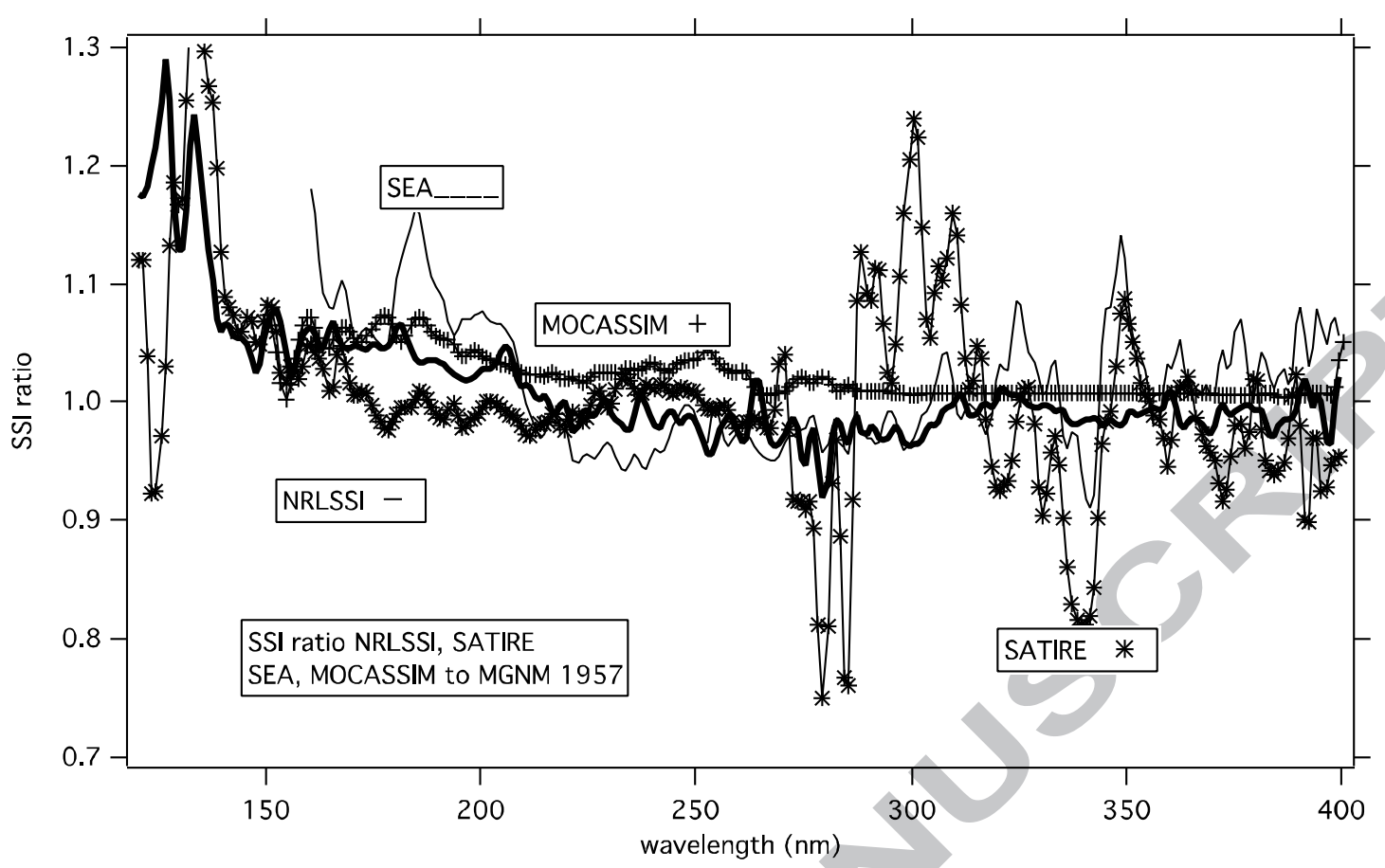

Figures 6a and 6b: SSI comparison for two periods of high and low solar activity using five reconstructions. The ratio of each predicted spectrum to MGNM is displayed.

Figures 6a,b illustrate the different predictions for years 1807 (Dalton minimum) and 1957 as typical examples for low and high solar activity.

Spectral features such as shown by SATIRE are kept whatever the level of solar activity is. In general the consistency of the prediction is higher for high solar activity. Furthermore, as a function of solar activity, some significant SSI features may appear.

\subsubsection{The Sun at solar activity minimum}

The solar minimum during the years 2008-2009, between solar cycles \#23 and \#24, was the longest solar minimum period since the beginning of space-based measurements, and there is considerable evidence that the Sun reached anomalously low levels of magnetic activity and EUV emissions. Lower than normal densities in the ionosphere and upper atmosphere have been documented, starting with Heelis et al. (2009) and Emmert et al. (2010). The relationship between these observations and solar EUV measurements and proxies is explored by Solomon et al. (2010, 2011, 2013). Although the interaction between solar EUV and geomagnetic effects on the atmospheric changes remains controversial, it is clear from diverse measurement sets that the thermosphere-ionosphere system was lower, cooler, and quieter than previous solar minima, and that the cause was ultimately solar in nature. These measurements, and various solar measurements and proxies, are reviewed in Solomon et al. (2013), where it is noted that the $\mathrm{F}_{10.7}$ index, Sunspot number, the Mg II core-to-wing ratio, $\mathrm{H}$ Lyman-alpha composite time series, and measurements by the SEM instrument on the SOHO spacecraft, all reached annual average values significantly lower than the previous minimum in 1996. However, thermosphere-ionosphere changes cannot be explained by the $F_{10.7}$ index alone, probably because of its tendency to reach a minimum value or plateau at a value of 
67; lower fluctuations of EUV emissions are not captured below the level at which $\mathrm{F}_{10.7}$ no longer has sensitivity to changes in radio emissions from solar active regions. Even worse, during the solar minimum in 1975 there were periods which in EUV and the radio emission $\mathrm{F}_{10.7}$ anti-correlate as observed independently from each other aboard the satellite missions Atmospheric Explorer-C (Hinteregger, 1977) and AEROS-B (Schmidtke et al., 1981) as summarized in Schmidtke (1985).

Since solar EUV radiation is produced in these solar active regions, it is not surprising that a solar minimum that was unusually long quiet magnetically as in years 2008 to 2009 should also produce less EUV. It is an open question as to the implications for other regions of the solar spectrum. Solar cycle \#24 has been weak as well as late, and is now in its declining phase. There has been some speculation that the Sun may be entering a period similar to the Dalton Minimum of the early 1800's, or even a new Maunder Minimum. How the solar spectrum changes on longer time scales is a central question for solar and space science, and provides significant motivation for long-term monitoring of spectral irradiance across the full extent of the solar spectrum.

When considering the TSI data from 1978, a significant minimum is easily detected occurring at spring in early June, 2008.

\begin{tabular}{cccc} 
& & & \\
& & & \\
\hline Spectra/solar activity & SSN & $\mathrm{F}_{10.7}$ & $\mathrm{Mg}$ II index \\
\hline ATLAS 3 & 18 & 80.0 & 0.26747 \\
SOLAR 2008 & 2.4 & 68.8 & 0.26426 \\
\hline
\end{tabular}

Table 1: For the ATLAS 3 and SOLAR, the level of activity of the Sun is shown with the Sunspot number (SSN), the radio electromagnetic flux at $10.7 \mathrm{~cm}$, and the $\mathrm{Mg}$ II index.

SOLAR being launched in February, 2008, we obtained the first light data in April. For UV measurements, it is frequently assumed that the first light data is not altered by environmental processes (e.g. contamination by exhaust material from ISS transport vehicles). In similar condition of solar activity, SORCE-SIM has generated a solar spectrum (Whole Heliospheric Interval, WHI, Woods et al., 2009), which we shall use for comparison. The importance of such spectrum is:

- It is the first time that SSI is measured with such a low solar activity,

- Models are frequently using such type of spectrum to which they apply a variability wavelength dependent to generate spectra at other levels of solar activity based on various proxies used by these models. 
ACCEPTED MANUSCRIPT

\begin{tabular}{ccccc}
\hline$\Delta \lambda(\mathrm{nm})$ & Lines/continuum & A3/SOL & WHI/SOL & WHI/A3 \\
\hline $17-120$ & See below & 1.06 & 0.95 & 0.90 \\
$15-25$ & Fe XVII, O VIII, Cr XV, Ca XIV, ... & & & 1.05 \\
$28-35$ & He II, Fe XVI & 1.09 & 0.75 & 0.69 \\
$55-65$ & He I, O VI & 0.95 & 1.26 & 1.33 \\
$96-100$ & C III, Ly $\gamma$, N III & 1.48 & 1.28 & 0.86 \\
$101-105$ & Ly $\beta$, O VI, C III & 1.05 & 0.87 & 0.83 \\
$121-122$ & Ly $\alpha$ & 1.42 & 1.38 & 0.96 \\
$166-180$ & continuum & 1.06 & 0.97 & 0.92 \\
$120-200$ & Continuum,O II, C II, Si IV, C IV, Si II & 1.06 & 1.00 & 0.94 \\
$180-220$ & continuum, Si II, Al edge & 1.03 & 0.95 & 0.92 \\
$170-207$ & continuum, Si II, Al edge & 1.04 & 0.98 & 0.94 \\
$207-300$ & deep Fraunhofer lines & 1.04 & 0.97 & 0.94 \\
\hline
\end{tabular}

Table 2: Ratio of SSI in specific wavelength domains for ATLAS 3 and WHI to SOLAR and WHI to ATLAS 3 below $200 \mathrm{~nm}$. The ratio in the 15-25 nm domain is not calculated as Using the SOLSPEC and SolACES data (named SOLAR), a spectrum has been built (Thuillier et al., 2014a). It is compared to ATLAS 3 and WHI in different spectral intervals. The Table 2 shows the results.

SolACES measurements are available for wavelength greater than $17 \mathrm{~nm}$. Data set names are abbreviated in the column headings as A3, SOL for ATLAS 3 and SOLAR, respectively.

We note that the SOLSTICE-SORCE data are calibrated at the NIST SURF III facility and use quiet blue stars to track relative changes in the instrument (Snow et al., 2014), whereas SolACES measurements are calibrated in the absolute scale using ionization chambers. These two techniques are independent. Furthermore, the ATLAS 3 spectrum was obtained when the sunspot number was 18, while SOLAR and WHI are representative of a sunspot number equal to 3 .

- The closest agreement between WHI and SOLAR is met within the range 17 to 120 $\mathrm{nm}$. Nevertheless, there are variations within that domain, which are shown below as a function of wavelength.

- WHI/SOLAR ratios of $0.75,1.26$ and 1.28 are reaching the limit of their combined uncertainties in the intervals $28-35 \mathrm{~nm}, 55-65 \mathrm{~nm}$ and $96-100 \mathrm{~nm}$ as well as at Ly $\alpha$. We note the presence of many emission lines in these intervals. Very likely, the explanation of such high ratios may be found in the PSF of each instrument in which the strong emissions lines are not identically placed. 
- In case where the solar continuum contributes more than the lines, the difference is not greater than $5 \%$, which is a number fully consistent with the instrumental uncertainties. This supports the interpretation of high ratios in presence of strong emission lines, as shown in the previous paragraph, rather than different instruments calibration accuracies.

- Comparing WHI and SOLAR with ATLAS 3 suggests, as expected, a higher ATLAS 3 SSI (Thuillier et al., 2004).

In conclusion based on this comparison the ratios such as 0.75 or 1.25 contains the combined uncertainties and the effect of the instruments PSF. Precise PSF is necessary to move forward in this study. Nevertheless, a mean difference reaching $20 \%$ below $100 \mathrm{~nm}$ represents a significant improvement with respect to datasets gathered in the 1980's. A detailed analysis of the solar spectrum at solar minimum activity may be found in Thuillier et al. (2014).

\section{Work in progress}

In this section on-going TIGER/COSPAR topics are presented with preliminary results.

In Figure 7 a first approach of a combined data set is presented using an EUV data set from the SDO/EVE and SOLAR/SolACES instruments covering the spectral range of 16.5-105.5 $\mathrm{nm}$ and a period of $3 \frac{1}{2}$ years. This data set is used for testing applications in ionospheric investigations as well as in other fields. Preliminary results are described.

\subsection{Elaboration of EUV SSI reference data}

One of the major tasks is the elaboration of EUV SSI reference data sets. As shown in Figure 7 a first approach of EUV SSI data is presented covering the spectral range of 16.5-105.5 nm and a period of 3.5 years. The solar flux is a combination of SDO/EVE and SOLAR/SolACES data relevant for ionospheric applications. 


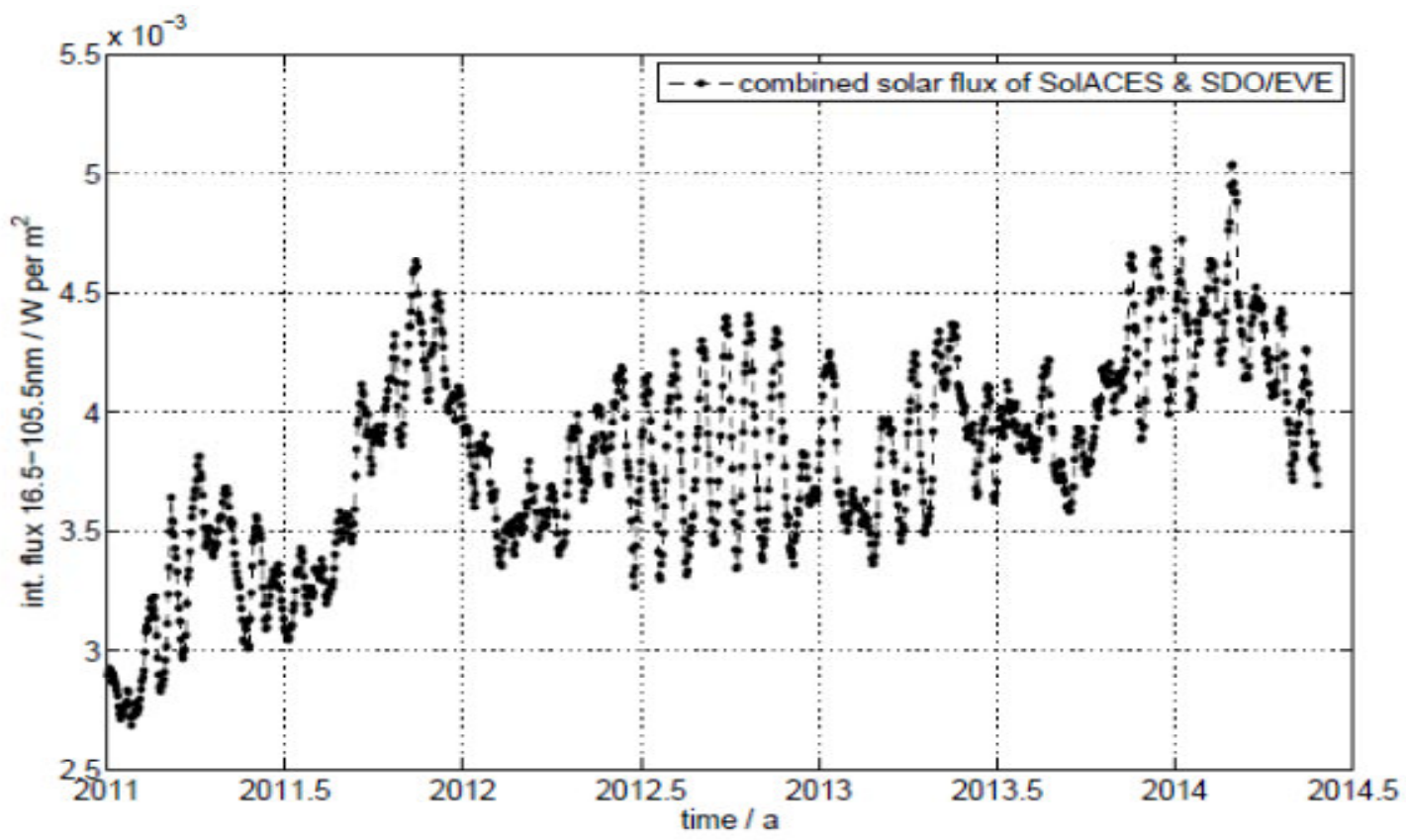

Figure 7: Combined EUV data set from the SDO/EVE and SOLAR/SolACES instruments covering the spectral range of $16.5-105.5 \mathrm{~nm}$ and a period of 3.5 years.

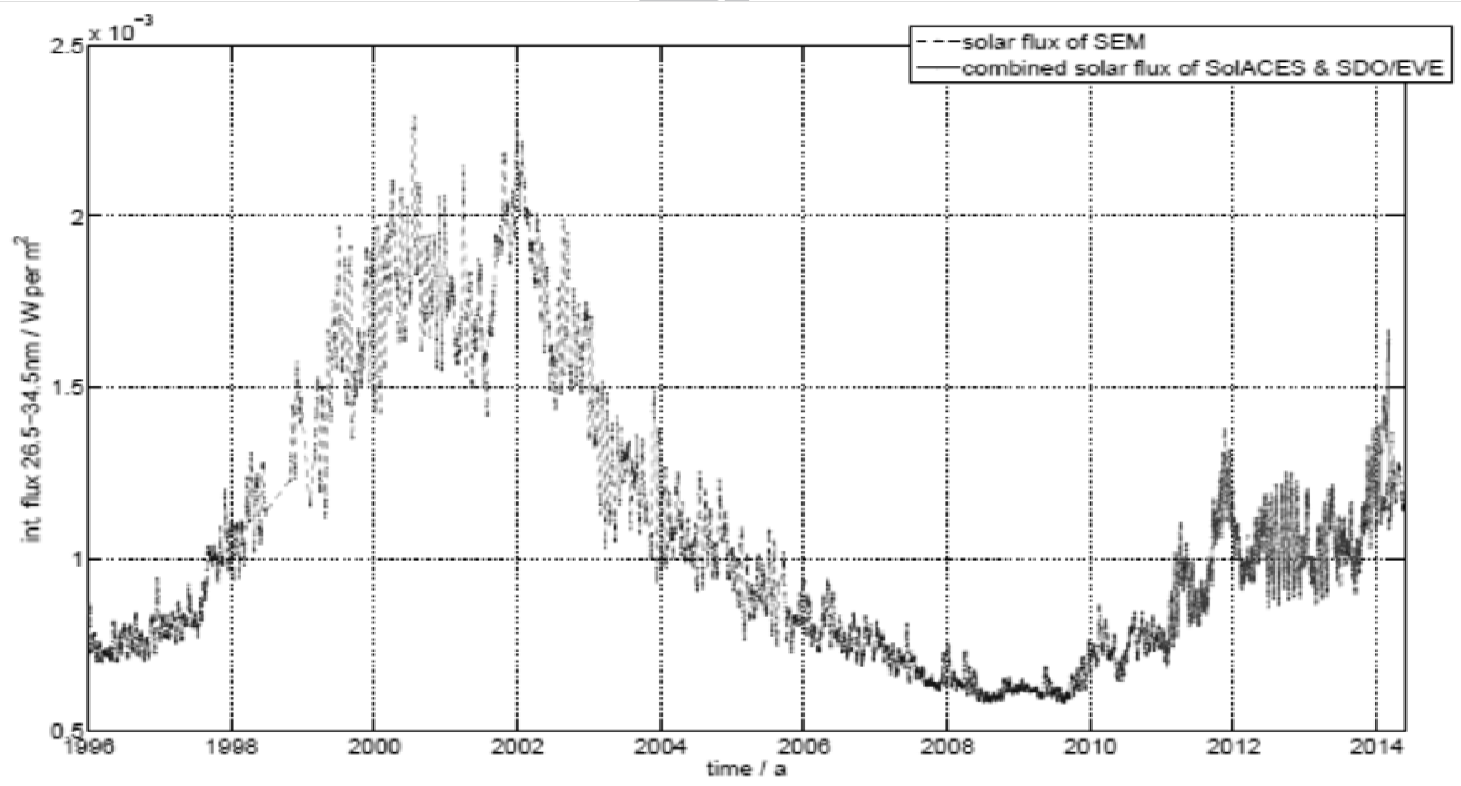

Figure 8: Inter-comparison of solar fluxes from SEM and combined EVE \& SolACES data of Figure 7 during cycles 23 and 24.

Extension of the spectral range up to $150 \mathrm{~nm}$ and of the period back to 2008 is also in progress taking into account data from SEE. Since measured EUV SSI data are available back 
to 2002 the aim is to elaborate a full set of validated EUV SSI data covering the spectral range of 16-150 $\mathrm{nm}$ for the period of a solar cycle (2002-2015).

Inter-comparison of EUV SSI data from SEM, SolACES, and SDO shows good agreement (see Figures 7 and 8). However, there is a significant deviation of the SEE data with time as shown in Figures 9 and 10. A possible explanation is given in Section 3.2.

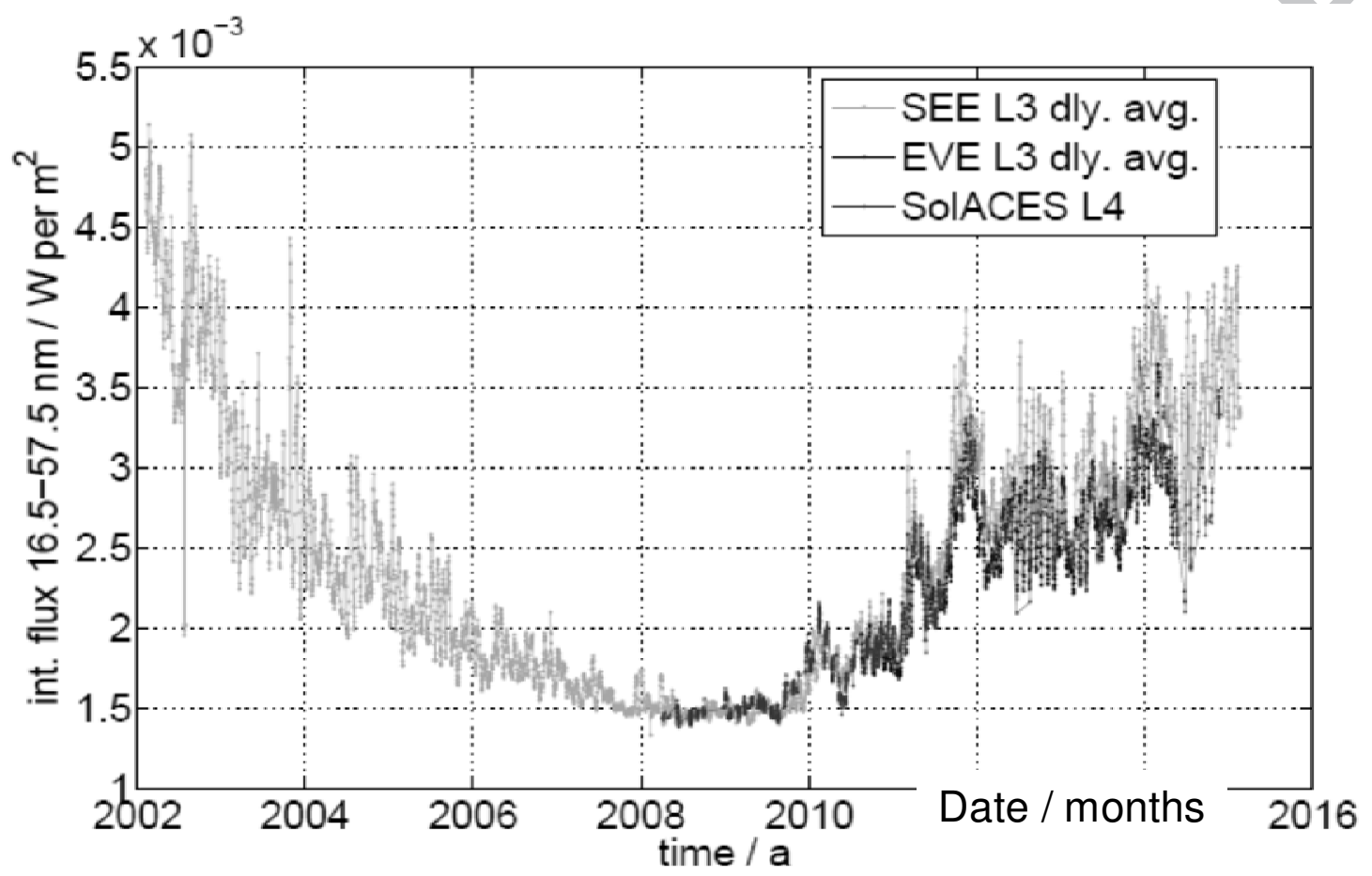

Figure 9: Inter-comparison of SEE, SolACES, and EVE data in the spectral range of 16.5$57.5 \mathrm{~nm}$.

During solar minimum between cycles 23/24 and during the onset of the solar cycle 24 there are unexpectedly large changes in solar EUV activity. The period of solar minimum is lasted significantly longer than the preceding ones. The onset of the solar cycle 24 was not as steep at lower intensity levels as observed during the preceding cycles. Also the maximum levels of cycle 24 are well below cycle 23 . 


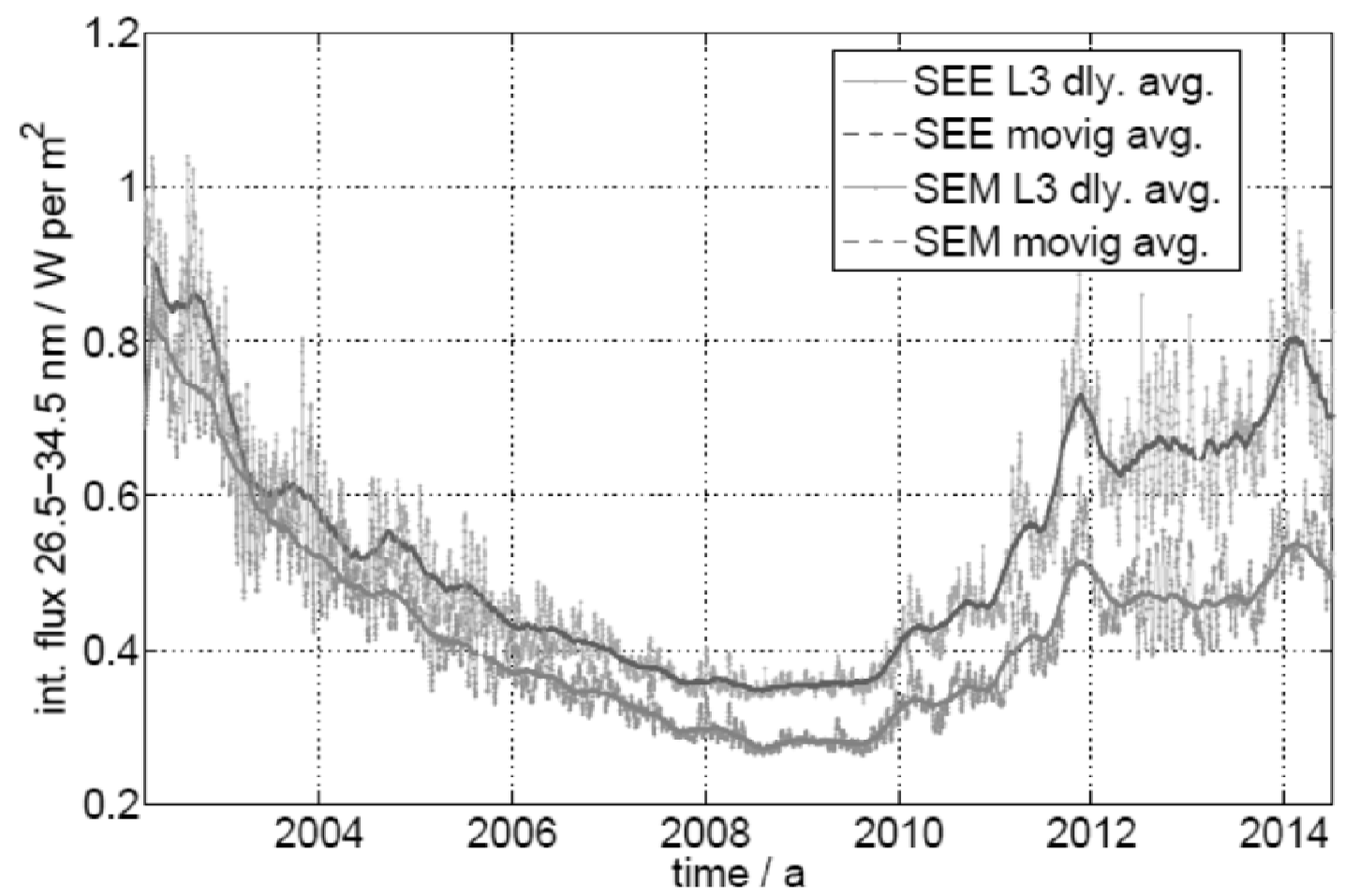

Figure 10: Comparison of SEE (upper part) and SEM (lower part) data in the spectral range of 26.5-34.5 $\mathrm{nm}$ with running mean of 137 days.

There is strong scientific interest to learn how the next solar minimum will develop raising questions such as whether the next solar minimum will again cause lowerthermospheric temperatures and ionospheric densities below past minima, and what the impact on long-term T/I climatology will be.

\subsection{Application of EUV SSI reference data in ionospheric research}

Ionization rates and plasma density are well correlated with solar irradiation and its variability. The ionization of neutral gas components starts at wavelengths less than about 130 $\mathrm{nm}$, where the photon energy exceeds the ionization potential of main thermospheric constituents such as $\mathrm{N}_{2}, \mathrm{O}_{2}$, O, and NO. Since dual frequency measurements using Global Navigation Satellite Systems (GNSS) provide the integral of the vertical electron density distribution up to GNSS satellite altitude of about $20,000 \mathrm{~km}$, referred to as Total Electron Content (TEC), such measurements are well suited to study the ionospheric response to solar activity changes (e.g. Davies et al., 1992). On the other hand, solar irradiation and its variation can seriously impact GNSS based navigation and positioning since the ionospheric range error is primarily proportional to TEC.

The main constituent of the F2 layer that contributes essentially to TEC is ionized atomic oxygen whose ionization potential is equivalent to the energy of photons at $91 \mathrm{~nm}$. Hence, spectral components at wavelengths less than $91 \mathrm{~nm}$ will strongly be associated with TEC 
measurements. Since TEC measurements are usually carried out at elevation angles less than $90^{\circ}$ providing so-called slant TEC (STEC) they must be converted to an equivalent vertical TEC (VTEC) to get a geometry-free reference. If data coverage is sufficient, as over Europe, VTEC maps can be constructed (e.g. http://swaciweb.dlr.de ) from which ionospheric range error corrections may be derived (Jakowski et al., 2011) for use in single frequency applications, such as performed in Space Based Augmentation Systems (SBAS) to enable precision navigation for aviation.

Since the solar EUV radiation in the wavelength range relevant to the ionosphere can vary by more than a factor of three from solar minimum to solar maximum (Hinteregger, 1981), it is expected that ionospheric TEC will follow the solar cycle. Indeed, this can be seen in Fig. 11. Here the solar irradiation is indicated by the proxy $\mathrm{F}_{10.7}$.

It is evident that the general behavior of TEC is strongly correlated with $\mathrm{F}_{10.7}$ and therefore is an important input parameter for ionospheric models (Jakowski et al., 2011; Hoque and Jakowski, 2011). This dependency is modulated by seasonal effects related to solar irradiation conditions that impact the direct ionization as well as thermospheric density and composition. Thus, TEC decreases with increasing geographic latitude as can easily be seen in Figure $11 \mathrm{~b}$.
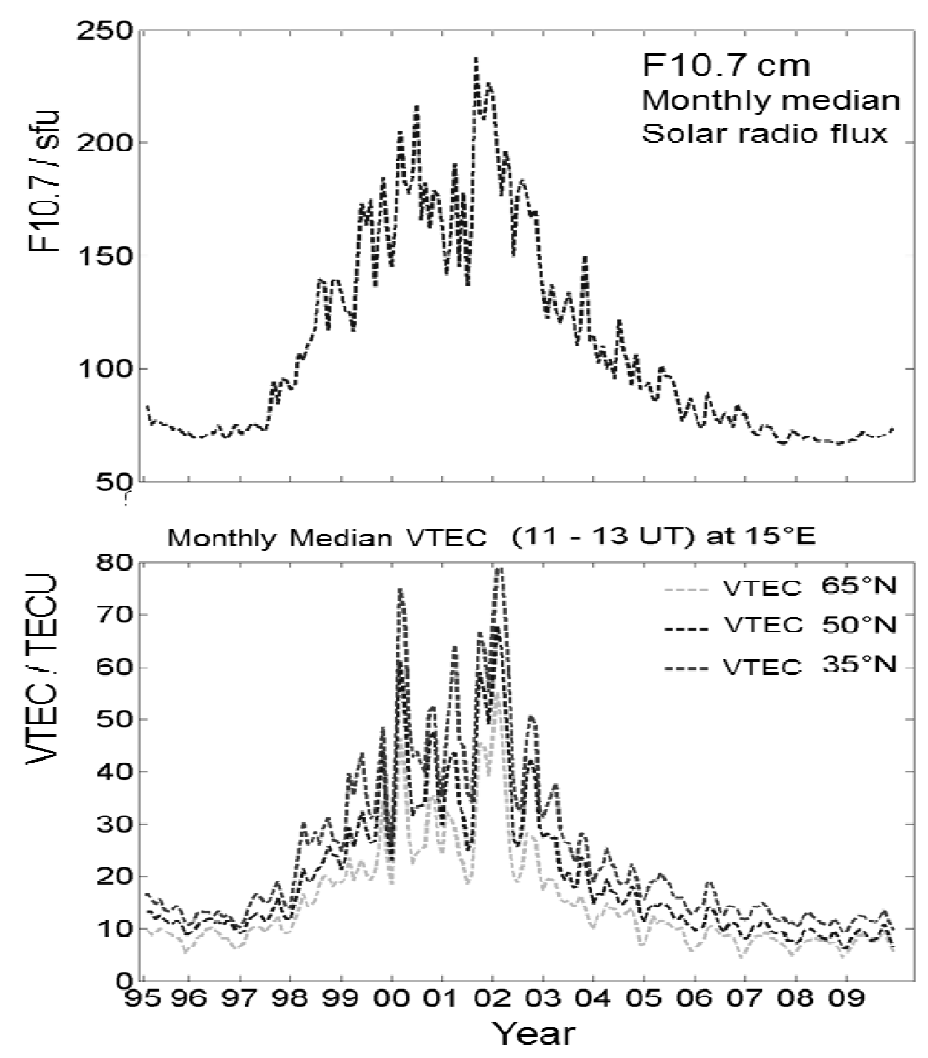
Figures 11a and 11b: Upper panel - Solar activity variation $\left(\mathrm{F}_{10.7}\right)$ from 1995 -2009 covering solar cycle 23 (1996-2008). Lower panel - Day-time variation of TEC averaged over 12-14 LT at three selected points in Europe. (1 TECU $\left.=1 \times 10^{16} \mathrm{~m}^{-2}, 1 \mathrm{sfu}=10^{-22} \mathrm{Wm}^{-2} \mathrm{~Hz}^{-1}\right)$.

TEC derived from GNSS over Europe has a dynamic ratio of about 8 for day-time average. This variability is primarily driven by two energy sources: The relevant solar EUV fluxes change by more than a factor of three. Taking into account that multiple ionization takes place in the spectral EUV range below $45 \mathrm{~nm}$ and that solar EUV fluxes below $30 \mathrm{~nm}$ are dominated by intense iron emissions changing even more than by a factor of three the primary and secondary photo ionization of atomic oxygen is increased correspondingly. In addition, electron fluxes and magnetospheric currents, especially during CME events, add to the TEC values thus increasing the dynamic ratio.

It is important to note that the ionospheric response to radiation changes associated with the 27 days rotation period of the Sun is delayed by about 1-2 days (Jakowski et al., 1991, Jakowski et al., 2002, Lee et al., 2012; see also Fig. 12).

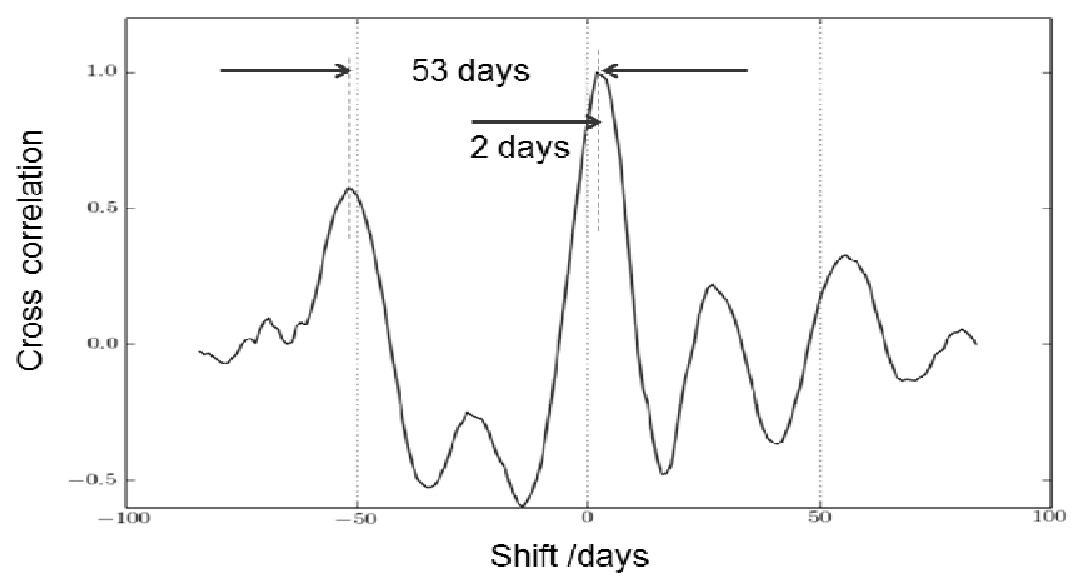

Figure 12: Cross correlation of SolACES+EVE data with VTEC obtained at $50^{\circ} \mathrm{N} ; 10^{\circ} \mathrm{E}$ within the period of Nov. 1, 2012 - Jan. 20, 2013.

Jakowski et al. (1991) estimated the ionization level by Faraday rotation measurements at VHF signals of the SIRIO- and SMS-1 geostationary satellites from which TEC was deduced. The cross correlation analysis with the solar radio flux index $F_{10.7}$ revealed a time delay of 1-2 days for measurements at the Neustrelitz receiving station. To explain the delayed response of the ionospheric ionization it was supposed that thermosphere-ionosphere coupling plays a significant role. This assumption was investigated in more detail by Jakowski et al. (1991) by 
modeling the thermospheric response to the 27 days solar radiation variation. Atomic oxygen that is generated by photo-dissociation is accumulated due to slow diffusion in the lower thermosphere. Due to the accumulation of oxygen atoms, the atomic oxygen number density variation lags the irradiance variation. Since the major ionization in the F2 layer is proportional to atomic oxygen density, the simulation results are consistent with the observed delayed response of VTEC.

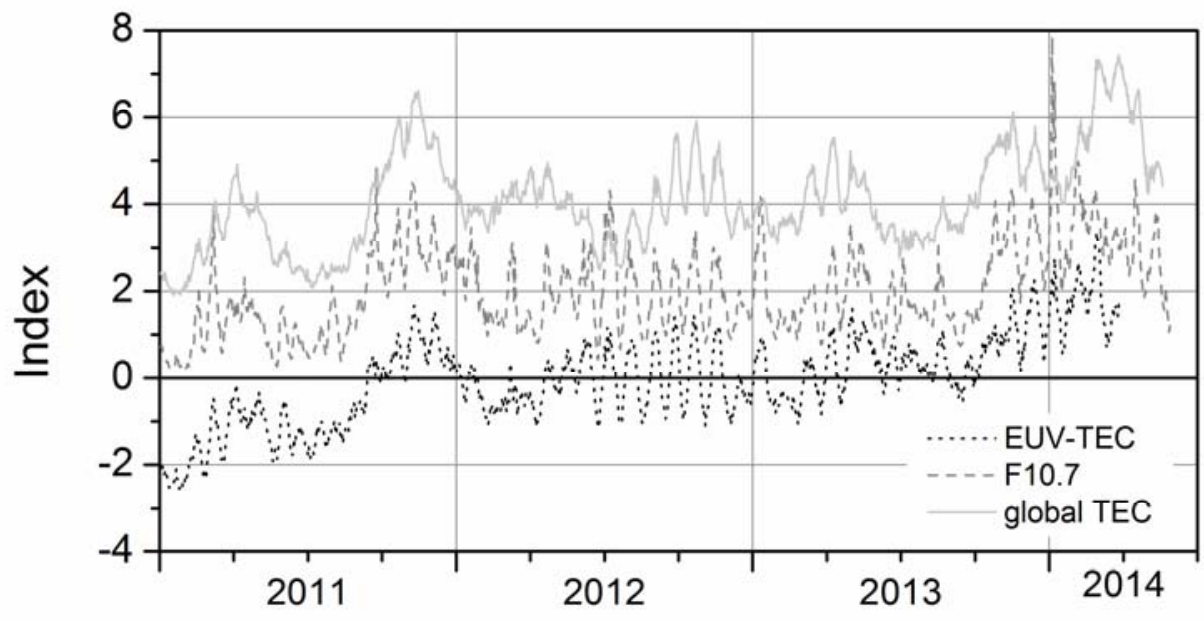




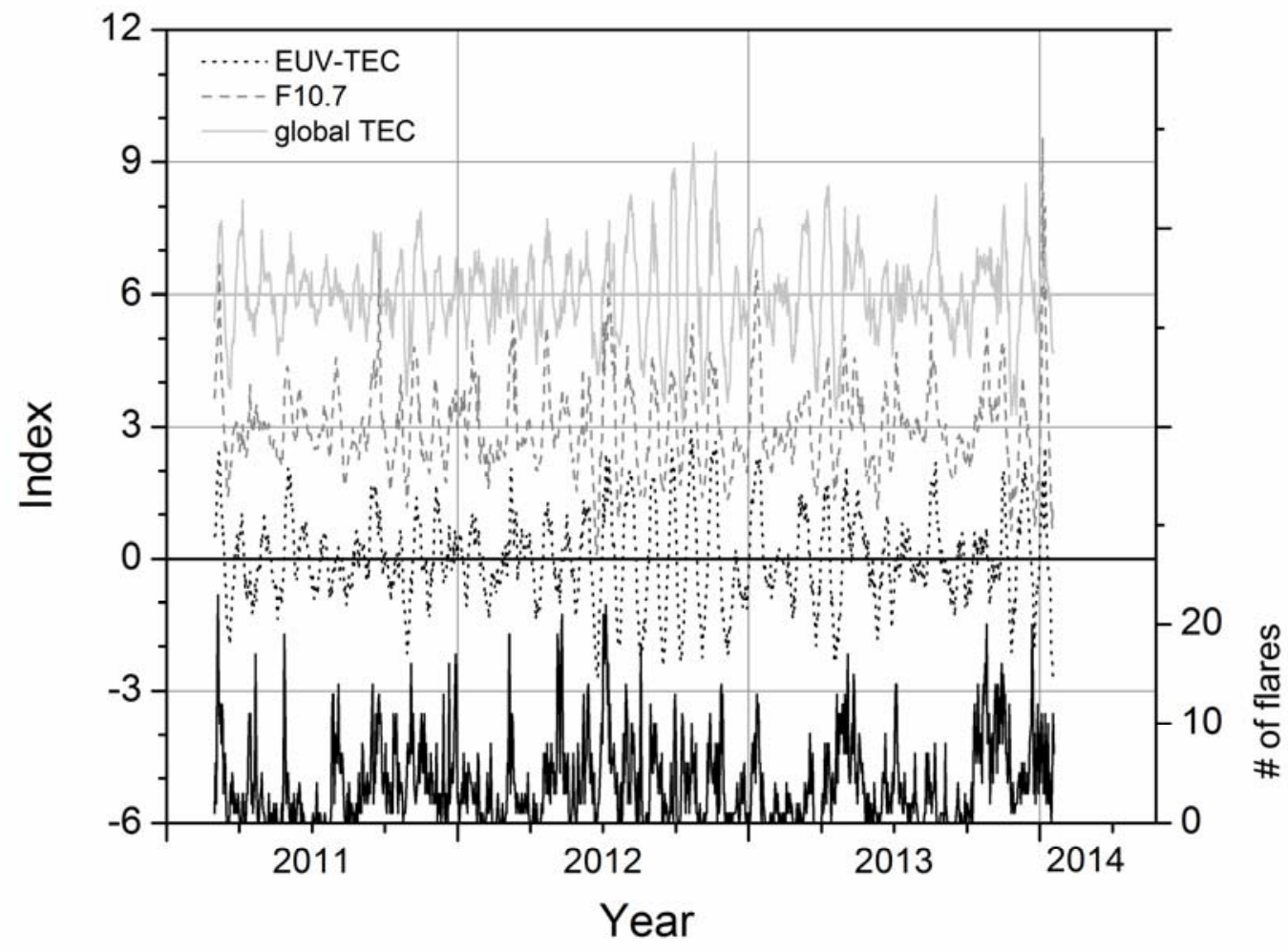

Figures 13a and 13b: EUV-TEC (I) - Upper panel: Comparison of combined EUV-TEC data (black dots) from SDO-EVE and SolACES with $\mathrm{F}_{10.7}$ (grey dashed) and global TEC (light grey). The curves have been shifted by +2 with respect to each other. Lower panel: High-pass filtered data; curves have been shifted by +3 with respect to each other. In the lower part the number of solar flares per day is shown. Data shown are daily sums of reported C, M, and X flares.

Nevertheless, more detailed studies are needed to verify this hypothesis and to fully understand the underlying physics in the thermosphere-ionosphere region. Due to the lack of proper solar radiation data, the $\mathrm{F}_{10.7} \mathrm{~cm}$ radio flux index has been used as a proxy in earlier studies. Now, with the availability of new sensors and satellite missions to measure EUV, the experimental situation is improved. A number of new capabilities also exist to measure the electron density distribution in the ionosphere and TEC using ground and space based GNSS measurements (Jakowski et al., 2002, 2011). Thus, Figure 12 shows the delayed response of TEC for $50^{\circ} \mathrm{N}$; $10^{\circ} \mathrm{E}$ within the time period Nov. 1, 2012 - Jan. 20, 2013 to SolACES+EVE data (16.5-105.5 nm; see Figure 7). TEC has been extracted from vertical TEC maps provided routinely by DLR via the SWACI ionosphere weather service (http://swaciweb.dlr.de). Figure 12 shows a modulation of TEC by the solar rotation period of 27 days and a delayed response to EUV radiation of about 2 days. 
Figure 13a shows global primary ionization rates that have been calculated using EUV data from combined EVE and SolACES measurements from 2011 to early 2014 (see Figure 7). The model includes ionization of $\mathrm{O}_{2}, \mathrm{~N}_{2}, \mathrm{O}$, and $\mathrm{N}$ after Unglaub et al. (2011) and the normalized results have been termed EUV-TEC. Normalized $F_{10.7}$ indices are shown in gray, for comparison. The light gray line indicates global mean TEC calculated from IGS TEC maps. Since the seasonal cycle of EUV radiation and TEC is different, the variance of the three parameters at the time scale of days to weeks is different, and interpretation is difficult. Therefore, in Figure $13 \mathrm{~b}$ we show the data filtered using an FFT filter with cut-off period of 3 months. There is a tendency for $\mathrm{F}_{10.7}$ extremes to overestimate TEC, more than EUV-TEC does. In the lower part of the figure, the number of solar flares per day is shown. Data shown are daily sums of reported $\mathrm{C}, \mathrm{M}$, and $\mathrm{X}$ flares.
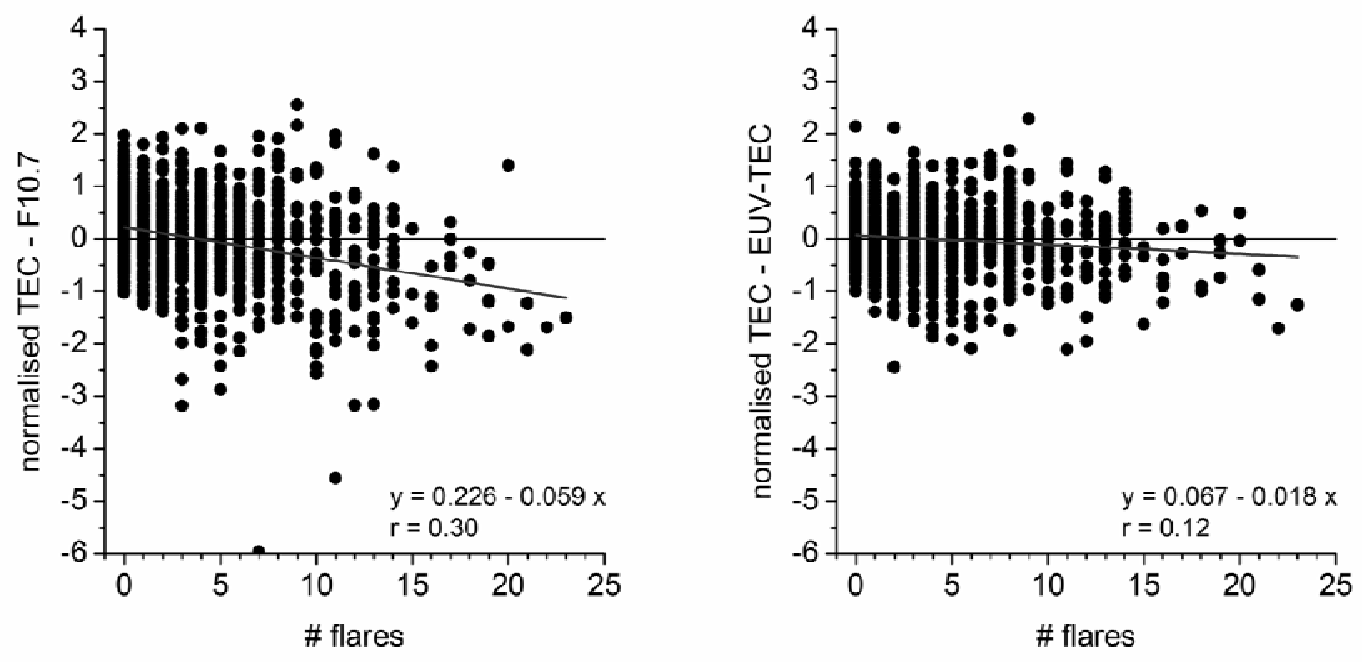

Figure 14a and 14b: EUV-TEC (II) - Differences between TEC and $\mathrm{F}_{10.7}$ (left) and EUVTEC (right) vs. the number of solar flares.

The differences between global mean TEC and $\mathrm{F}_{10.7}$ and EUV-TEC are shown in Figures $14 \mathrm{a}, \mathrm{b}$ vs. the number of solar flares as a proxy for short-term activity. The figures show that $\mathrm{F}_{10.7}$ values are too large to describe TEC variations for large number of flares (negative values of TEC $-\mathrm{F}_{10.7}$ in the left panel), while EUV-TEC better describes the TEC variability for both quiet and disturbed Sun.

There is still considerable variability of TEC not explained by EUV-TEC. Part of that may be due to time shift between TEC and EUV-TEC/ $\mathrm{F}_{10.7}$, which is visible in Figures 13 for a local scale.

These initial results are subject to further investigation:

- Cross correlation of SolACES+EVE data (16.5-105.5 nm; see Figure 7) with TEC provided by the DLR Space weather service SWACI at $50^{\circ} \mathrm{N} ; 10^{\circ} \mathrm{E}$ within the time period Nov. 1, 2012 - Jan. 20, 2013. 
- Results indicate a modulation of TEC by the solar rotation period of 27 days and a delayed response of ionospheric TEC by about 2 days.

- This result confirms previous cross correlation studies between TEC and the $\mathrm{F}_{10.7} \mathrm{~cm}$ radio flux.

The time shift is expected to depend on the zenith angle of the incoming solar EUV flux with respect to the equator and on solar activity. The zenith angle changes with season from 0 to $+/-23 \mathrm{deg}$. Then the absorbed EUV energy is hitting different magnetic field lines at $+/-23^{\circ}$ and $0^{\circ}$ such that the distribution of the photo electrons and the distribution of the energy are carried out along different ways and altitudes. Solar activity affects the energy distribution in two ways. First, the altitude regime of the energy deposition is lower at solar minimum and higher at solar maximum. In addition, the energy content of the solar spectral irradiance shifts toward shorter wavelengths with increasing activity, so high energy photo electrons increase by more than a factor of two compared to low energy photoelectrons.

To study the possible correlation of $\mathrm{F}_{10.7}$ and EUV-TEC with regional values of TEC two sets of stations covering mid latitudes in Europe and Africa have been considered. 16 stations ranging from $52^{\circ}$ to $67^{\circ}$ magnetic dip angle have been taken into account for Europe and 6 stations ranging from $-52^{\circ}$ to $-64^{\circ}$ magnetic dip angle for Africa. TEC values were obtained from the corresponding RINEX files using the technique described in Ciraolo et al. (2007). Figures $15 \mathrm{a}$ and $15 \mathrm{~b}$ show the results for a period of four solar rotations where $\mathrm{F}_{10.7}$ and EUVTEC indices in arbitrary scales are compared with a plot of all TEC values from the European and African stations respectively. In all cases it appears clear that at the regional level both indices are not sufficiently correlated to TEC. Only in the fourth rotation does the EUV-TEC index appear to be slightly more correlated with TEC than with $\mathrm{F}_{10.7}$. These results indicate that the present indices alone cannot be used for practical applications. Further studies are necessary to assess how to improve EUV indices in view of potential applications for regional or local purposes. 


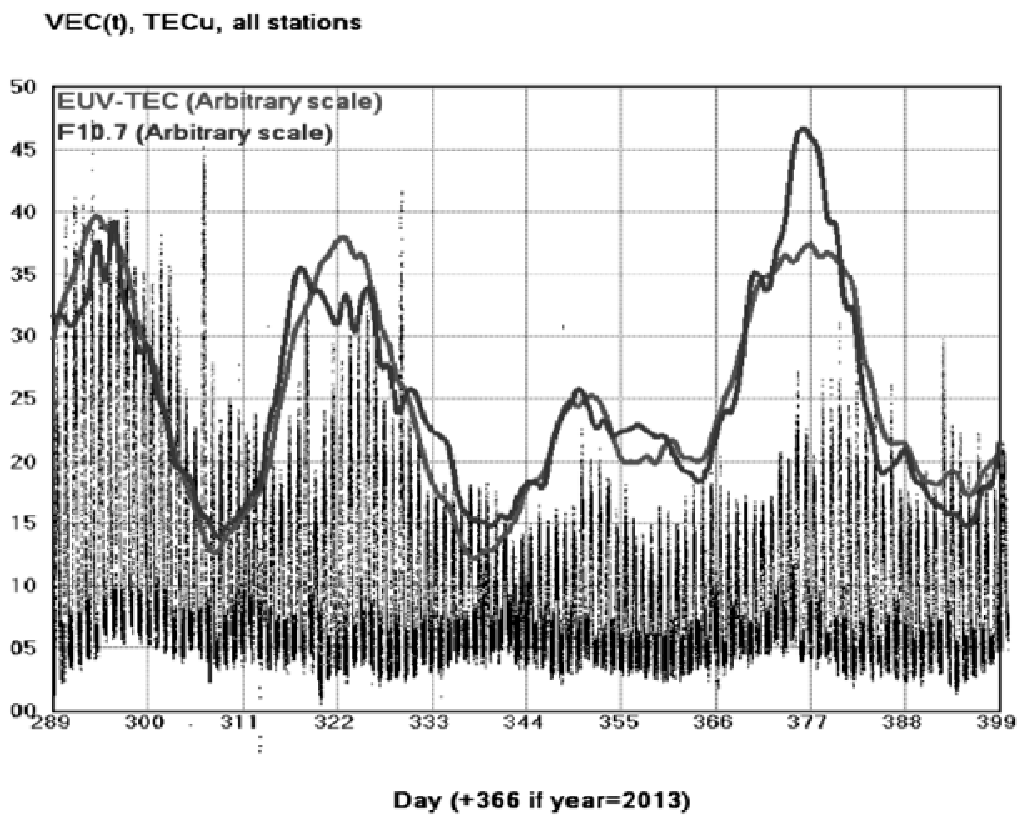

Figure 15a: Four solar rotation time series of $\mathrm{F}_{10.7}$ and EUV-TEC indices (in arbitrary scales) compared with the mass plot of TEC from 16 ionospheric middle latitudes stations in Europe.

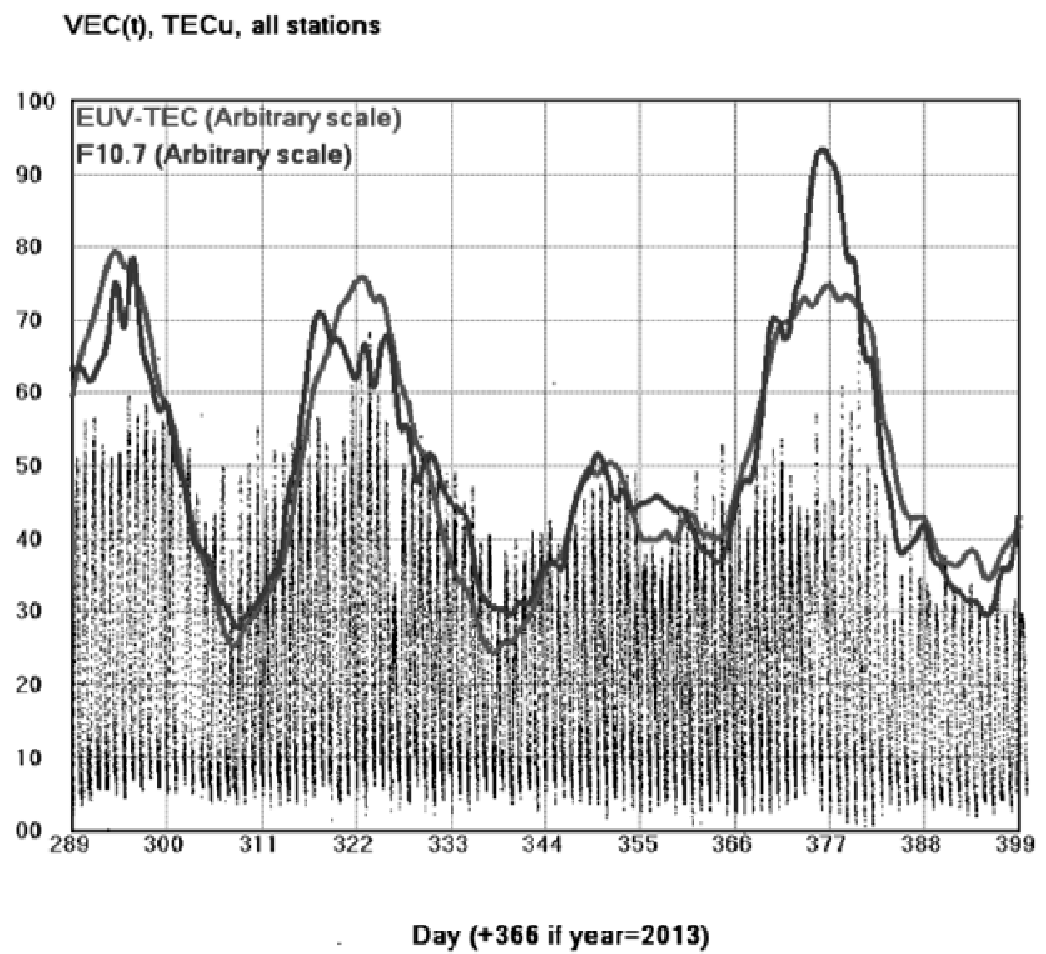

Figure 15b: Four solar rotation time series of $\mathrm{F}_{10.7}$ and EUV-TEC indices (in arbitrary scales) compared with TEC from 6 ionospheric middle latitudes stations in Africa. 


\subsection{Application of EUV/UV SSI reference data for testing EUV/UV SSI models}

As we obtain increasingly precise EUV and UV data, theoretical work continues significant improvement in understanding the physical processes of the solar emission. A major difficulty arises with efforts to compare theoretical spectra given at high spectral resolution with measurements at low spectral resolution. Integration of the theoretical spectra in the instrument bandpass is made, however, precise knowledge of the bandpass is provided, but the bandpass could change in time once the instrument is in space. The possibility of wavelength-dependent degradation remains a significant difficulty for space instrumentation.

\subsection{Does the occurrence of solar minima depend on wavelength?}

EUV data from SEM, SolACES, and SOLSPEC show indications that the date of solar minima depends on wavelength.

For example, SEM measurements in the spectral range of $26-34 \mathrm{~nm}$ provide $5.85 * 10^{-4} \mathrm{~W} / \mathrm{m}^{2}$ on day 218 of year 2008 (05-AUG-2008) and $5.84 * 10^{-4} \mathrm{~W} / \mathrm{m}^{2}$ on day 233 of year 2009 (21AUG-2009). This later 2009 date for solar cycles 23/24 minimum is supported by SolACES recordings (see Figure 16).

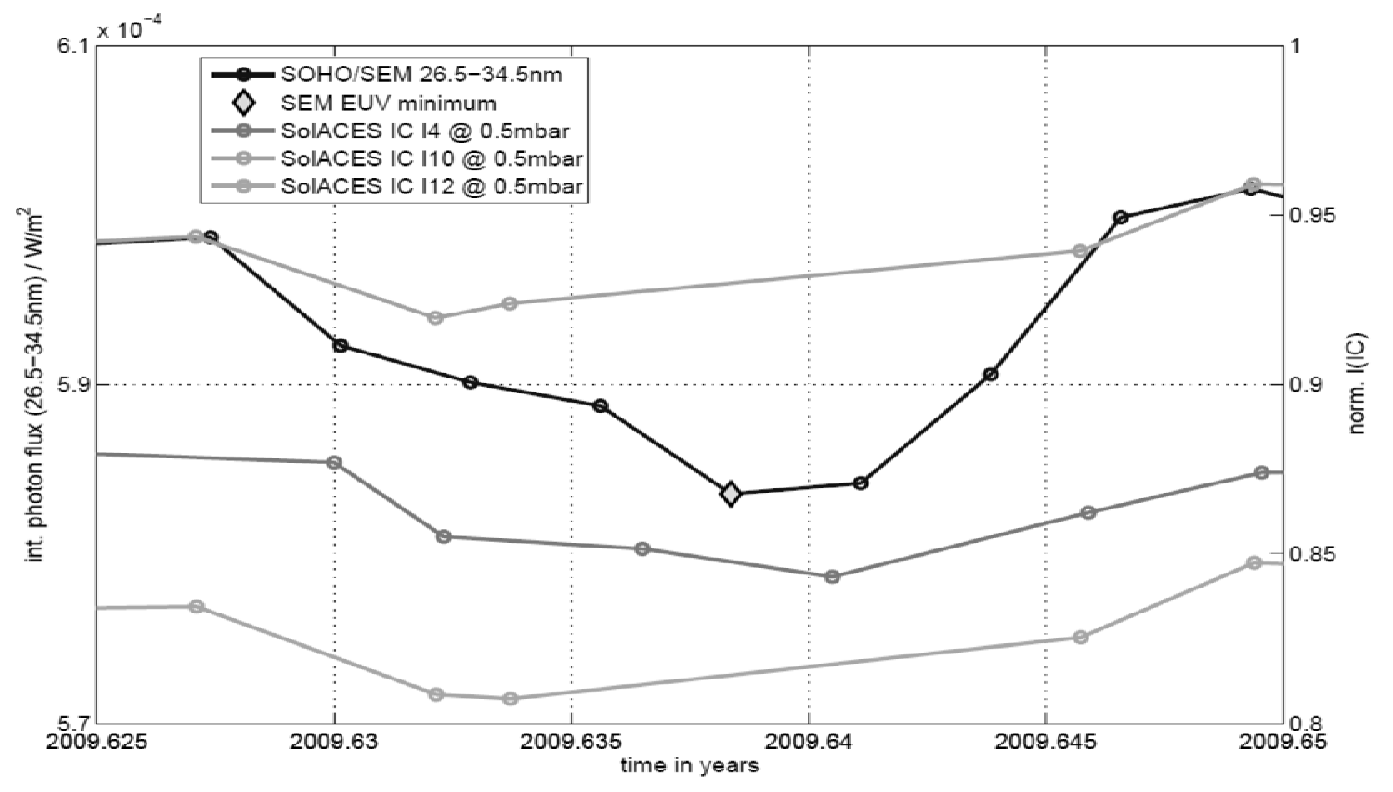

Figure 16: SEM and SolACES data during the solar minimum between solar cycles 23/24.

Figure 17 presents the SSI variability at $215 \mathrm{~nm}$ as measured by SOSTICE. Without ambiguity, the UV solar minimum occurs about one year before the EUV minimum. With respect to the SSN minimum occurrence, the delay is largest for the highest altitude of emission. 
It should be kept in mind that the EUV/UV minima occur at different times with respect to the time of change of the magnetic field alignment in the Sunspots as first noted by Hinteregger (1977) and confirmed by Schmidtke et al. (1981).

SOSTICE data show the occurrence of the minimum in the UV around end of April, 2008 (Figure 17) while Sunspot numbers (SSN), the radio electromagnetic flux at $10.7 \mathrm{~cm}$, and the Mg II index indicate minima in early June, 2008 (see Table 1).

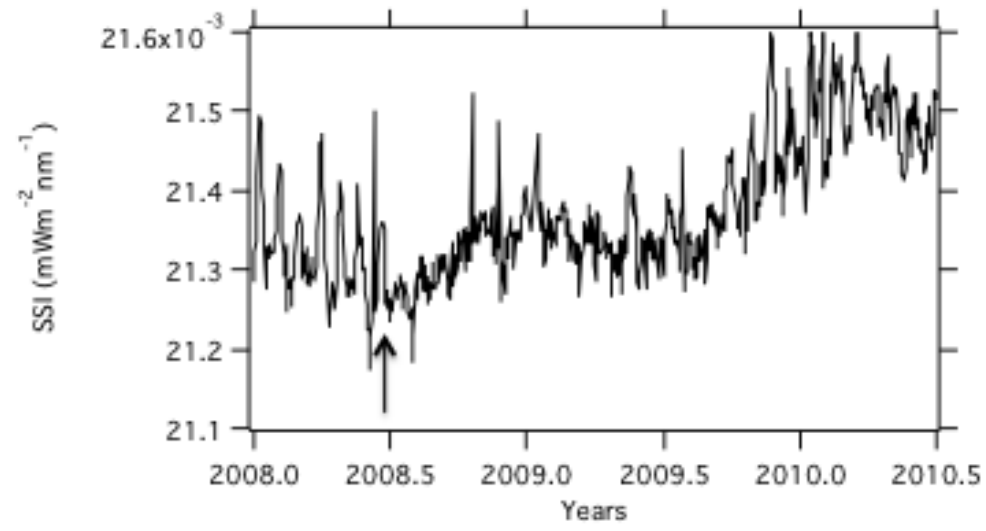

Figure 17: SSI at $215 \mathrm{~nm}$ measured by SOLSTICESIM

It should be noted that the radiometric accuracy of EUV/UV data can be complicated by the subtle trends in instrument degradation corrections, so it may not yet allow confirming the assumption of solar minima appearing at different times depending on wavelength. Therefore, increasing the accuracy of SSI data and improved techniques for tracking instrument degradation are mandatory to further investigate this topic.

\section{Proposed TIGER/COSPAR topics}

The following topics are considered substantial for the future TIGER/COSPAR activities.

\subsection{Continuous measurement, verification, and compilation of EUV/UV SSI data}

As described in Section 3 current measurements deliver EUV/UV data covering the full wavelength range of interest. However, most of the instruments are suffering from instrument aging effects. Also from the operational point of view we are approaching technical failures with high probability as instruments continue on past their design life. If next-generation instrumentation is not implemented very soon, it is highly questionable that we can have overlapping SSI data to derive an accurate long-term record of solar EUV/UV variability. For example, there is high interest in comparing the long-term solar changes of the unusual last solar minimum with the approaching one that might be 2019 .

The SSI data recorded up to now need to be verified and compiled in a common open source data storage and combined into a composted long-term record of solar variability. This 
process has started in the UV with a record back to 1978. In the EUV a first approach has started as discussed in this publication. It will be pursued in the frame of the TIGER/COSPAR initiative in close cooperation with the SOLID team.

\subsection{Developing instrumentation and methods for making a radiometric standard for calibrating and cross-calibrating EUV/UV SSI data in space}

After the problem of aging in the laboratory and even more in space became obvious different methods were and still are applied towards improving the SSI data evaluation. For example, with a radioactive source (nickel 63 with 92 years half-life) producing 64-keV beta particles the efficiency of magnetic electron multipliers was traced (Schmidtke et al. 1974). The efficiency changes recorded already reached a factor of two from the synchrotron calibration to the first measurement in space (Schmidtke et al., 1975). Underflight of calibration rocket experiments have provided a more accurate cross-calibration of space instruments (Woods and Rottman, 1990; Schmidtke et al., 1992; Worden et al., 1996). Internal cross-calibration of redundent channels of same the production history but with different measurements of exposure rates to solar radiation in space has also proven useful for tracking instrument trends (Woods et al., 2005). Using ionization chambers as a primary detector standard helps for recalibrating EUV spectrometers in space (Schmidtke et al., 2014). In the UV spectral regime UV light sources are applied to re-calibrate spectrometers (Thuillier et al., 2009). - These instruments with in-flight calibration capability (e.g., SOLSPEC and SolACES) could be duplicated for the next generation of XUV/UV/VIS/IR measurements.

As we have learned about the advantages and disadvantages of these methods the next step is selecting instrumentation and calibration techniques to provide the most accurate SSI data. In this context the accuracy of the three-current ionization chambers shall be further improved in the course of a doctorate work by investigating the impact of higher energetic EUV/XUV photons that generate secondary electrons and/or Auger electrons in the ionization chamber.

\subsection{Measurement of airglow/auroral EUV/UV radiation and developing instrumentation and missions for real-time monitoring of space weather phenomena}

Each terrestrial EUV/UV airglow emission is generated by specific physical processes in the T/I system, such as by photo-electron impact excitation, resonant and fluorescent scattering, ionization and dissociation, radiative recombination (Schmidtke et al., 1993). The physical modeling of recorded airglow data is quite complex due to the manifold changes within the T/I system, hence such as changing altitudes where the absorbed solar EUV/UV SSI energies are deposited due to solar activity, and due to daily and seasonal changes of T/I parameters with variable solar energy input from above and lower atmosphere dynamics from below.

The measurement of EUV/UV airglow emissions aboard the spinning satellite San Marco 5 (Schmidtke et al., 1985, 1991) disclosed intensity distribution of the equatorial region differing with atmospheric altitude and wavelength while recording nadir and limb airglow spectra from nadir to zenith angles while the satellite changed orbital altitude from $619 \mathrm{~km}$ down to about $200 \mathrm{~km}$ at the end of the mission in 1988. The TIMED Global UltraViolet 
Instrument (GUVI) has been observing the nadir and limb FUV airglow over the full globe since 2002 and during the time of the interesting solar cycle minimum in 2008-2009.

Several new space flight missions currently in development will use airglow emissions to infer thermosphere-ionosphere properties, and so are reliant on knowledge of solar spectral irradiance, particularly in the EUV and soft X-ray regions. The NASA Global-scale Observations of the Limb and Disk (GOLD) mission will be carried as a hosted payload on a commercial communications satellite in a geostationary orbit. It images temperature and $\mathrm{O} / \mathrm{N}_{2}$ composition on the sunlit disk of the Earth by analyzing FUV emissions from the $\mathrm{N}_{2} \mathrm{LBH}$ bands and the $\mathrm{O}\left({ }^{5} \mathrm{~S}\right)$ doublet at $135.6 \mathrm{~nm}$. It also measures temperature and $\mathrm{O}_{2}$ density on the limb, and, at night, images the equatorial ionosphere, including regions depleted by ionospheric instabilities. The NASA Ionospheric Connection Explorer (ICON) is a low-Earth orbit satellite in a near-equatorial orbit that will investigate the interaction between atmospheric and solar influences on space weather. It carries a Michelson interferometer to measure winds from oxygen airglow emissions at 630.0 and $557.7 \mathrm{~nm}$, an EUV spectrometer to image ionospheric density from the $\mathrm{O}^{+}$triplet at $83.4 \mathrm{~nm}$, a FUV spectrograph for composition from $\mathrm{N}_{2} \mathrm{LBH}$ and $\mathrm{O}\left({ }^{5} \mathrm{~S}\right)$, and an ion velocity meter. Both missions will launch in 2017. Additional data from international constellation missions such as COSMIC-2 and SWARM will vastly increase our understanding of the global thermosphere-ionosphere response to changes in space weather and climate.

These missions have analysis strategies designed to retrieve the required parameters in the absence of any information concerning solar spectral irradiance, but clearly their goals will be enhanced by solar observations. For example, understanding the thermosphere-ionosphere response to flare events would be limited unless there is a reasonable estimation of the flare intensity and spectral character. On longer time scales, if these new missions can endure for a solar cycle, as has the NASA TIMED mission, the systematic seasonal and cyclical variation in the upper atmosphere will be better characterized through comprehensive measurements of solar spectral variability.

From the manifold airglow emissions available the bright line emitted from $\mathrm{O}^{+}$at $83.4 \mathrm{~nm}$ is particularly interesting for real-time observations of space weather phenomena. Four types of ionospheric disturbances can be distinguished, CME and solar wind driven disturbances, daily day-night turbulences, equatorial super bubbles, and those caused by solar flares. 


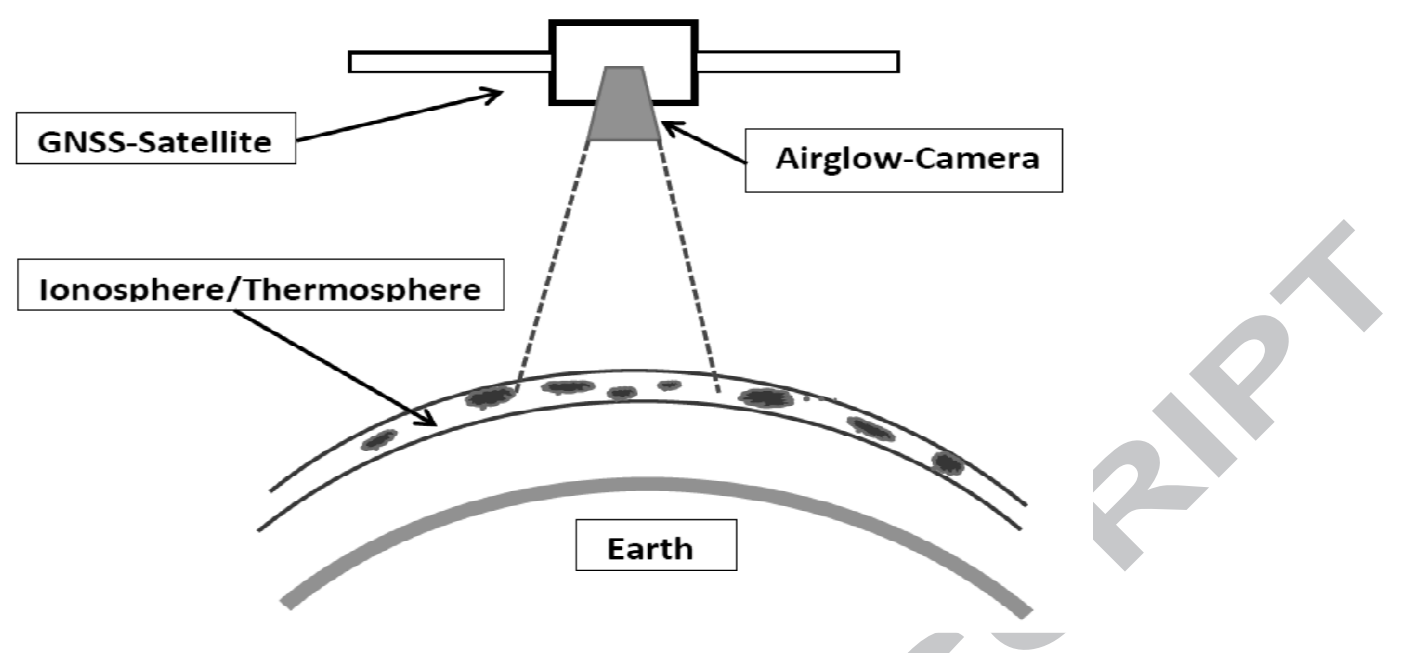

Figure 18: Position of an airglow space weather camera (SpaWeCam) aboard a GNSS satellite

Airglow space weather camera (SpaWeCam) could be placed aboard multiple navigation satellites (Figure 18). Supposing a field of view (FOV) of $+/-5$ deg for a three-mirror shortfocal-length telescope and with an array detector to provide spatial resolution of 5 arcmin, then the nadir view would have a resolution equivalent to $\sim 33.5 \mathrm{~km}$ for studying the $\mathrm{T} / \mathrm{I}$ system. For example, if the SpaWeCam was integrated aboard a Galileo satellite at an orbit altitude of $23.260 \mathrm{~km}$ the FOV corresponds to a circular field of observation of $\sim 4000 \mathrm{~km}$ diameter at T/I airglow emission altitudes.

An estimate of the signal-to-noise ratio $(\mathrm{S} / \mathrm{N})$ for observing the $\mathrm{O}^{+}$emission at $83.4 \mathrm{~nm}$ with a maximum photon flux of 800 Rayleigh is derived using a primary ring telescope mirror with an outer diameter of $40 \mathrm{~cm}$ and an inner diameter of $15 \mathrm{~cm}$ and assuming a 200x200 pixel channel-plate detector with an EUV-VIS converter coupled to a CCD-camera. The estimate of the $\mathrm{S} / \mathrm{N}$ leads to $2 \times 10^{4} \mathrm{cps}$ per pixel. With the 14-hour orbit period of a Galileo spacecraft, one would need to take make an image every 40 seconds to achieve the $33 \mathrm{~km}$ resolution, and only 10 of the 30 operational Galileo spacecraft would need to have SpaWeCam to provide global coverage over a day.

The data evaluation is explained for allocating a SpaWeCam aboard Galileo satellites. The assignment of geographic coordinate pairs (longitude and latitude) of the T/I system, e.g. at $200 \mathrm{~km}$ altitude for the $200 \times 200$ pixels, can made with the GNSS attitude data in combination with the angular/geometric pixel parameters of the SpaWeCam. This way the signal of each pixel is directly mapped to the T/I area. For the investigation of space weather phenomena the data of the double-matrix (intensity and geographic coordinates at a certain level of altitude) can be transmitted to ground in two steps, first during an in-orbit calibrating and measuring period and, second during a measuring and re-calibration period.

In-orbit calibrating and measuring period:

Parallel to the on-board data recording, GNSS signals received at ground in GNSS receiving stations are evaluated to provide total electron content (TEC) information that 
can be correlated with the airglow signals of the SpaWeCam transmitted to ground for calibration purposes. From these data a rather simple series of correction parameters can be created to be sent to the GNSS satellite to improve their navigation accuracy. The correction factors are sorted according to the degree of disturbing GNSS signals e.g. by numbers from 0 to 20 representing discrete magnitudes of TEC values. This way the strength of the airglow signals is correlated to the degree of disturbed GNSS signals controlled by TEC values.

Measuring and re-calibration period:

This airglow-TEC comparison provides disturbance factors containing 200x200 geographic coordinate pairs plus disturbance values from 0 to 20 for the range of TEC values. These factors can be transmitted with the GNSS signals for correcting the navigation data at each geographic location and thus providing even more accurate navigation information. For re-calibration purposes the efficiency of the pixels can be checked and corrected for airglow instrument aging by repeatedly deriving the correction factors at multiple ground stations. Therefore, ground stations are not required in uninhabited or restricted regions.

This proposed concept with SpaWeCam airglow measurements from Galileo constellation of satellites has a couple of scientific advantages too. For one, the SpaWeCam data can provide quantitative evaluation of space weather disturbances from particle contributions that can then be used with EUV SSI in T/I modeling. In addition, the integration of the measured EUV/UV airglow fluxes over the globe will provide improved understanding of the contribution of EUV/UV airglow and albedo to the upper atmosphere energy balance.

\subsection{Investigation of relations between solar EUV/UV and VIS/NIR emissions}

It is important to improve our understanding of the relationship between solar EUV/UV and VIS/NIR emissions, for several reasons. First, such knowledge may help to constrain the physical processes that cause these emissions on the Sun. For example, it has long been known that the emissions from various parts of the solar atmosphere are often nonlinearly correlated, which can be used to study the energy balance and typical geometrical properties of the emitting regions on the Sun (e.g., Hammer and Schleicher 2000). And second, if a large database of such correlations between EUV and VIS measurements is available, it might be possible to bridge short gaps, where for some reason no EUV data are available from spaceborne instruments, with the help of ground-based VIS data. Such indirect methods can never be perfect, because of the variability of solar activity, but it could be useful as a rough approximation in cases where no direct EUV measurements are available. Thus we consider it important to study relationship between EUV/UV and visible/NIR variability. However, this is a signifcant challenge because the solar visible/NIR variability is below the threshold of the detectability of the current space instruments. 


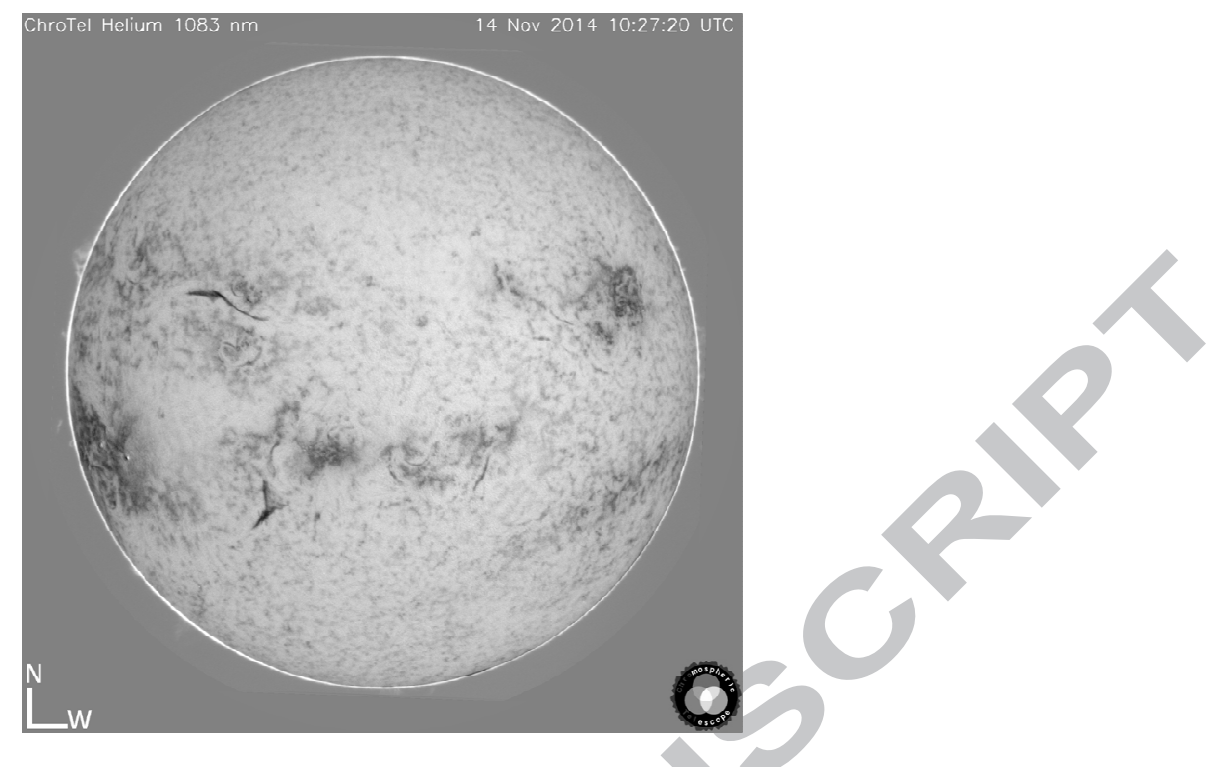

Figure 19: Full-disk image of the Sun, taken in the neutral He line at $1083 \mathrm{~nm}$ (ChroTel telescope on Tenerife, KIS)

For these reasons, we suggest to support space-borne observations with ground-based solar image measurements. This requires a telescope that observes the entire solar disk as often as weather conditions and observatory operation permit. These requirements are met by ChroTel, a full-disk telescope (Bethge et al., 2011) operated by KIS in the Observatorio del Teide on Tenerife. The telescope provides narrow-band images of three chromospheric lines, namely $\mathrm{H} \alpha, \mathrm{Ca} \mathrm{K}$, and the He I 1083.0 nm line. The He I filter is tunable, and observations are taken at seven wavelength positions covering the whole spectral line. The image cadence is 3 minutes, and the spatial resolution is 2 arcsec. Figure 19 shows an example, taken on November 14, 2014, a day with rather high solar activity. The picture is taken at the center of the solar absorption line, thus dark areas indicate regions where the line absorption is strong, and the brightest areas show only a very weak absorption or no line at all. It is obvious from visual inspection that the Helium line depth is a marker for magnetic activity. A quantitative analysis will be able to distinguish between filaments (they appear black in Figure 19, i.e., the He line is very strong there), and other magnetically active areas, like spots or plages. Although ChroTel operates mostly during the summer and autumn months, there are monthlong periods of daily data that will allow a quantitative comparison of $\mathrm{He} 1083$ data with EUV data and with the Sunspot numbers.

It is of particular interest to compare the He I $1083 \mathrm{~nm}$ line, which is mostly formed in the upper solar chromosphere, with the resonance lines of neutral and singly ionized helium, 58.4 $\mathrm{nm}$ and $30.4 \mathrm{~nm}$, respectively, both formed in the lower parts of the transition region between chromosphere and corona. Because of the very high excitation energies of these lines, the respective energy levels can be populated either by collisions with sufficiently energetic electrons, or by photoionization with energetic photons and subsequent recombination. The relative importance of these mechanisms has been debated for about four decades. Currently 
most authors believe that the 30.4 line is mostly collisionally excited, the $1083 \mathrm{~nm}$ line mostly by photoionization/recombination, and the $58.4 \mathrm{~nm}$ line by a combination of both effects. The relative importance of the excitation mechanisms depends also on the region on the Sun. Moreover, the resonance lines are not only very important for the energy balance of the solar transition region, but they also have several properties that differ from other lines formed in the lower transition region (like their response to temporal variations). These differences have been ascribed in the past to numerous effects, like turbulent mixing, non-Maxwellian electron contributions, diffusion effects across the magnetic field, or even abundance variations. These issues are not yet completely solved. We hope that we can contribute more information towards a better understanding of the most important emissions from the second most abundant element on the Sun.

\subsection{Modeling solar EUV/UV SSI data for different applications}

Knowledge of the EUV and FUV solar flux and its variability is also a key problem in the framework of planetary space weather. Various processes such as ionization or dissociation triggered by the solar flux leads to atmospheric emissions, especially in the UV and visible spectral ranges. Barthelemy and Cessateur (2014) have shown the critical impact of the uncertainties of UV measurements on the estimates of the atmospheric parameters regarding planets, moons and exoplanets. As an example, proxy models of the EUV leads to an underestimation of about $15 \%$ on the Total Electron Content (TEC) within the Ganymede atmosphere (Cessateur et al., 2012a). It is feasible that the models of the EUV/UV SSI could be improved with the more recent solar EUV/UV observations and thus could improve upon the research concerning terrestrial and planetary atmospheres.

\subsection{Developing solar EUV/UV indices for different applications}

In many applications, $\mathrm{F}_{10.7}$ is used as a proxy for EUV and its variability, however, it could be shown that a) using measured EUV fluxes or indices such as S10, M10, and Y10 rather than the $\mathrm{F}_{10.7}$ proxy provides a (somewhat) closer correlation with ionospheric TEC F, E, and D regions and b) there are further processes influencing ionospheric electron density and subsequently positioning errors such as the delay shown in Figure 12. Therefore, to further improve ionospheric models such a delay must be taken into account in new EUV indices. This means, they must be based on EUV measurements, but includeing a time shift especially at the 27-day time scale. This time shift, which is both time scale and regionally dependent, can be obtained from either extensive data analyses (e.g., using TEC maps in combination with radio occultations to obtain a 3-dimensional global electron density picture), or from careful global ionospheric modeling including thermospheric dynamics.

\subsection{Modeling the thermosphere/ionosphere with EUV SSI data}

Following a recent Chapman Conference of the American Geophysical Union, the Monograph Modeling the Ionosphere-Thermosphere System (Huba et al., 2014) was published with contributions from many model developers in the ionosphere-thermosphere field. This monograph provides a detailed description of the physics of the system in 
conjunction with numerical techniques used to model it, and issues of current interest, including the interaction between the response of the IT system to forcings from the lower atmosphere and from solar and magnetospheric sources. Many well-known global general circulation models, ionospheric models, and airglow process models are described and validated in this Monograph, so an additional review will not be attempted here. Rather, we reiterate that although lower atmosphere interactions are currently of great scientific interest, solar forcing of the thermosphere-ionosphere remains paramount. There are effective methods to apply solar inputs to general circulation models (e.g., Solomon and Qian, 2005), and a general convergence between solar EUV and FUV observations and the latest editions of proxy models often used in modeling. However, some fundamental issues remain unresolved. Of these, the most significant is probably the question of how much solar energy is supplied by the soft X-ray region, especially in the $\sim 1 \mathrm{~nm}$ to $\sim 10 \mathrm{~nm}$ region, and how variable it is. These photons ionize the lower ionosphere E-region. Ion density in this altitude range around $110 \mathrm{~km}$ sets the stage for tidal forcing and variability imposed by the lower and middle atmosphere, which controls the equatorial electro-dynamo, and hence ionospheric morphology in the low-latitude anomaly region. Identifying the reasons why models, using current estimates of solar soft X-ray and H Lyman-beta, fail to obtain high enough levels of E-region ionization, is an unsolved problem for solar and ionospheric physics; it is possible, for example, that the dissociation of $\mathrm{O}_{2}$ by SRC $160 \mathrm{~nm}$ photospheric emission near 100-110 $\mathrm{km}$ (represented by the M10 index) may contribute to further ionization processes. Spectrallyresolved solar measurements in this region are a challenge, and a key area for future observations.

\subsection{Developing instrumentation and missions for EUV/UV SSI real-time and in-line measurements}

Following the discussions in Sections 3.2 and 3.4 as well as related to Figures 9 and 10 the implementation of space instruments with absolute in-flight capability providing SSI reference data of the highest possible radiometric accuracy is an indispensable need for future missions. Though validated real-time spectrally resolved SSI observations are not yet available, the current progress promises more accurate data sets within shorter time periods possibly even in real-time and in-line (e.g. for use in T/I models). There are some alternatives for different applications and requirements (see Section 2):

- T/I, mesosphere, and climate modeling: Spectrometers covering SSI from XUV to IR spectral regions with real in-flight calibration as provided by ISS-SOLAR. If crosscalibration capability becomes available, a compilation of instruments out of the following missions will fulfill the requirements explained in the preceding sections: SOHO, TIMED, GOES-13/-15, SDO, P-2, and CORONAS-PHOTON with EUV/UV spectrometers, broadband detectors (silicon detectors with thin film filters; Spherical EUV and Plasma Spectrometer (Schmidtke, 2015)), and/or poly-chromators.

- T/I, mesosphere, and solar physics: High-resolution EUV/UV spectrometers with high cadence of spectral recording and availability of frequently repeatable cross-calibration action. Instruments like SDO/EVE covering the XUV/EUV/UV spectral range are 
potential candidates - again cross-calibration capability must be available for recalibrating each channel of the detector array individually.

- T/I: Combination of low-cost EUV SSI broad-band detectors (silicon detectors with thin film filters; Spherical EUV and Plasma Spectrometer) or poly-chromators and EUV SSI modeling capability - also with availability of frequently repeatable cross-calibration action.

- Space weather: Availability of a space weather camera with cross-calibration capability from ground.

The instruments of the missions mentioned are verified in space except for the Spherical EUV and Plasma Spectrometer (SEPS). The latter one consists of a metallic sphere surrounded by two spherical grids providing three photoelectron currents generated by the absorption of EUV photons. Sweeping the voltage of the inner sphere the currents from the metallic sphere and the two grids provide measurements of the EUV photon flux. The data evaluation is similar to the one of the three-current ionization chambers of SolACES. SEPS was tested in the SolACES test chamber, in the ESTEC plasma chamber, and at the BESSY synchrotron in Berlin. It is space-qualified by vibrational testing (Schmidtke, 2015). - The metallic sphere is expected to be very stable with respect to long-term efficiency changes.

It is necessary to optimize the instrumentation to be selected with respect to costs with mission operations included, availability, accuracy of the SSI data, and data evaluation. Since modeling of EUV/UV SSI plays a major role acquiring data sets without gaps, the state of the art and an outlook are presented below.

With reference to the models, current proxy models (mainly using $\mathrm{F}_{10.7}$ ) are not as accurate as they need to be in estimating the solar EUV/UV variability for space weather purposes. However, direct spectrally resolved observations of the EUV part of the solar spectrum are still a real challenge because of instrumental degradation issues.

Numerous studies suggest that the solar spectral variability in the UV can however be described by only a few terms (Dudok de Wit al. 2005; Amblard et al. 2008). This result is the consequence of the remarkable coherency in the spectral UV irradiance variability, for which the origin comes from the strong structuring of the solar atmosphere by the magnetic field (Domingo et al. 2009).

Using this coherency property, Cessateur et al (2011) have shown using a statistical approach that the solar EUV/UV SSI can be reconstructed using a few carefully chosen spectral bands. A new instrument design using typically less than 5 spectral bands is then compliant for reconstructing the whole UV spectrum with a relative error of about $20 \%$. Such instrumental concept, using new detector and filters technologies is also suitable for planetary space weather purposes with a low mass and low power consumption (Cessateur et al. 2012a,b). Along with the increasing interest for nanosatellites for scientific purposes, this instrument design paves the way for future solar EUV/UV observations suitable for the short term modulation as well as for the long-term.

The development of a space weather camera for airglow monitoring is ready to be started. 


\subsection{Investigation of the role of X-rays in the upper atmosphere}

Physical modeling of the processes of ionization and optical excitation in the terrestrial upper atmosphere encounters on-going issues in understanding energetic balance in the Earth's aeronomy especially for short-term events caused by solar X-rays during flares. Actually it means that our knowledge of the main input parameter-values of the solar fluxes is still insufficient. Unfortunately there are no reliable models of energetic characteristics of the Sun's EUV and $\mathrm{X}$-ray fluxes during the solar flares of different intensity levels (the flare classes) and over the different phases.

Though the "ground state" of the ionosphere and thermosphere (I/T) is determined by the flux of solar EUV and soft X-ray radiation as the dominant global energy source for them, the I/T models commonly use proxy data to represent solar activity instead of the more accurate EUV/UV SSI data. Moreover, modeling the variable state of the I/T system is hampered in response to changes both in the solar irradiance and in the energy received from the magnetosphere, with the latter causing the more extreme variability in the state of the system. - Particle fluxes are excluded in TIGER because this complex topic is dealt with by other communities.

SDO and GOES satellites have recorded thousands of flare events in the EUV and soft X-ray regions. The increase of solar ionizing fluxes during solar flares causes the increase of the energy absorption in the atmosphere. In this case the relative magnification of solar fluxes have the maximum in the short wavelength spectral range below 2-4 $\mathrm{nm}$, while the absolute increase of these fluxes is completely determined by the long wavelength (EUV) part of the spectrum of ionizing solar radiation. However, for strong solar flares, the contribution of $\mathrm{X}_{-}$ ray radiation to the additional ionization and to the increase of excitation of UV emission of upper atmosphere exceeds the contribution of the increased EUV radiation. This fact is connected with the main role of X-ray solar radiation in the generation of ionospheric photoelectron and Auger-electron spectra - the principal factor of additional ionization and UV-excitation (Avakyan, 1998; Solomon et al., 2001; Bailey et al., 2002). The principal gap in the experimental determination of the ionospheric and upper atmospheric response on the solar flares is the absence of the quantitative measurements of this additional effect. For example, there is often full radio wave absorption during solar flares; therefore, it is rara to obtain the experimental radio data about the flare growth of the ionization for different ionospheric regions.

The upper atmosphere dayglow in the UV-spectral range (for example, in 135-160 nm, that is, the Lyman-Birge-Hopfield system of $\mathrm{N}_{2}$ bands) is determined by the direct process of the excitation by electron impact and thus show the direct response to the growth of solar ionizing flux during a flare. Concurrent solar and airglow measurements during a flare are also rare. The intensity of atmospheric emissions during solar flares can increase by more than a factor of up to 1.5 in observations at nadir, and by a factor of up to 10 at various altitudes. The contribution of the secondary ionization due to photoelectrons during optical class $2 \mathrm{~B}$ flares is predominant when compared with the direct photoionization at all heights from 90 to $180 \mathrm{~km}$ (Kudryashev and Avakyan, 2000). At the same time, only a combined experiment providing simultaneous measurements of the solar ionizing flux, UV-dayglow emissions, and of the 
ionospheric photoelectron spectrum will allow verification of models predicting the response of the upper atmosphere to a solar flare. So far, no simultaneous measurements of the solar spectral irradiance and the global scale character of the atmospheric and ionospheric composition and dynamics have been made.

The main point for future research is to better understand the photoelectron spectrum and its changes during a solar flare. Each solar photon in the X-ray spectral region shorter than 3.1 $\mathrm{nm}$ produces a positive doubly charged ion and two electrons: a photoelectron and an Auger electron. Thus two ion-electron pairs are generated in the ionosphere by the absorption of one $\mathrm{X}$-ray photon at wavelengths shorter than $3.1 \mathrm{~nm}$. Therefore, the contribution of the direct photoionization in the E-region during flares can increase by $25-35 \%$. These results on the rates of the additional ion formation were obtained by an accurate calculation of the entire photoelectron spectrum for energies lower than $600 \mathrm{eV}$ (Avakyan and Kudryashev, 1984a,b).

\section{Summary and conclusions}

Results from on-going missions demonstrate good progress in obtaining EUV/UV SSI data. However, the SSI data sets published show significant deviations from each other at some wavelengths. Many versions are published with lengthy publication delays, and there are still uncertainties with respect to the absolute levels of radiometric accuracy. This situation reflects the technological difficulties inherent in SSI spectroscopy and understanding instrument degradation trends, as well as leaving T/I modelers uncertain between using actual EUV/UV SSI data instead of solar activity proxies.

SOLAR/SOLSPEC and SOLAR/SolACES instruments aboard the International Space Station constitute the first SSI-related payload with auto-calibrating capability covering the solar spectral regions from the IR through the XUV.

In view of the current state we are now in a position to begin a new era in this field of research:

- We are now able to compile a full set of validated UV/EUV SSI data covering more than a full solar cycle for scientific and for technical applications with physical thermospheric/ionospheric modeling included.

- We acquired the know-how for future UV/EUV SSI data measurements at high accuracy levels.

- We can provide SSI data from IR to XUV spectral regions for modern climate modeling with inter-comparison of total solar irradiance (TSI) with integral solar spectral irradiance (SSI) data included.

In order to realize these possibilities the following projects are proposed with respect to priorities:

Installation of instrumentation into space that provide real-time, calibrated EUV/UV SSI data.

- The EUV/UV SSI monitoring requirements depend on the field of application (e.g. low-cost instrumentation using EUV modeling for T/I modeling; instrumentation with high spectral resolution and high cadence of data recording for solar physics). 
- Development of a composite EUV/UV SSI record using a combination of measurements and models to provide daily estimates of the SSI back to the 1970s.

- Continued implementation of solar indices such as the S10, M10, and Y10 for daily space weather operations are derived from EUV/UV SSI data.

Because there is no substitute for measuring SSI directly with highest possible accuracy, the installation of a verified reference cross-calibrating EUV/UV space instruments could:

- save a lot of resources still invested in the 'determination' of aging effects,

- allow more time devoted to science, and

- assure users that they are receiving validated data sets for their various applications.

In view of the limited resources available and keeping in mind that we are now able to make a big step forward we shall go on coordinating all activities as effectively as possible for the TIGER program.

Consequently, it is essential to ensure SSI measurements in the future for the following reasons:

- The recent solar cycle 24 differs significantly from other ones.

- It is expected that the next solar cycle could also be different (lower activity).

- SSI data can neither be predicted nor can be modeled reliable without real data.

- T/I modeling and other application require the availability of on-line SSI data.

In this context the following TIGER/COSPAR topics are proposed:

- Continuing research-quality measurements of the solar and airglow/auroral EUV/UV radiation,

- Validating proven EUV/UV space instrumentation and calibration techniques added by the development of new EUV/UV space instrumentation and calibration techniques to further improve the accuracy,

- Modeling of the solar EUV/UV radiation,

- Elaborating and further developing EUV/UV indices such as F10, F30, S10, M10, Y10, EUV $, E_{\mathrm{y}}, \mathrm{EUV}_{\mathrm{yz}}$, EUV-TEC, EUV-THERM, EUV-F ${ }_{10.7}$, EUV-CHARGING...

- Modeling of the thermosphere/ionosphere with EUV SSI data normalized to $F_{10.7}$

- Verifying the accuracy of the current EUV/UV data for solar-terrestrial applications,

- Providing real-time monitoring of EUV/UV SSI for space weather operations and use in T/I models,

- Continuing inter-comparison of total solar irradiance (TSI) with integral solar spectral irradiance (SSI) data from the soft X-ray through the infrared spectral regions,

- Monitoring of airglow/auroral EUV/UV radiation (e.g. by a space weather camera) for improving GNSS navigation accuracy and for space weather applications,

- ,Coordinating world-wide solar and terrestrial EUV/UV SSI studies and operations, 
- Establishing a shared TIGER data system for EUV/UV SSI data distribution and realtime streaming, and

- Drafting an experts report on securing support for future measurements.

Based on today's experience, we can move forward in acquiring and providing EUV/UV SSI data at high radiometric accuracy for a myriad of $\mathrm{T} / \mathrm{I}$ research and space weather applications.

\section{Acknowledgements}

TEC data for construction of global TEC maps have been provided by NASA through ftp access on $\mathrm{ftp}$ ://cddis.gsfc.nasa.gov/gps/products/ionex/. $\mathrm{F}_{10.7}$ indices have been provided by NOAA, NGDC through http://www.ngdc.noaa.gov/stp/space-weather/solar-data/. Solar flare numbers were provided by NOAA, Space Weather Prediction Center (SWPC), through http:// www.swpc.noaa.gov/ftpdir/indices. The SolACES team acknowledges the sponsoring and support by DLR Bonn, by ESA/ESTEC Noordwijk, and by the Fraunhofer Institute for Measuring Technique Freiburg. NCAR is sponsored by the U.S. National Science Foundation. The research leading to these results has also received funding from the European Communitys Seventh Framework Programme (FP7/2007-2013) under Grant Agreement no. 313188 (SOLID, http://projects.pmodwrc.ch/solid/).

\section{References}

Amblard, P., Moussaoui, S., Dudok de Wit, T. The EUV Sun as the superposition of elementary Suns. A\&A, 487, L13, 10.1051/0004-6361:200809588, 2008.

Avakyan, S.V. Auger effect in the optics of the upper atmosphere. J. Opti. Technol.. 65, 11, 410, 1998.

Avakyan, S.V., Kudryashev, G.S. The excitation and ionization at the upper atmosphere during solar flares with consideration of the photoelectrons. Geomagn. Aeronomy 24, 4, 693-695, 1984a.

Avakyan, S.V., Kudryashev, G.S. The spectrum of photoelectrons in the upper atmosphere of Earth during the solar flares. Cosmic Research 22, 6, 889-894, 1984b.

Barthelemy, M., Cessateur, G. Sensitivity of upper atmospheric emissions calculations to solar/stellar UV flux. J. Space Weather Space Clim., 4, A35, http://dx.doi.org/10.1051/swsc/2014033, 2014.

Baum, W. A., Johnson, F. S., Oberly, J. J., Rockwood, C. C., Strain, C. V., Tousey, R. Solar Ultraviolet Spectrum to 88 Kilometers. Phys. Rev. 70, 781-782, DOI: http://dx.doi.org/10.1103/PhysRev.70.781, 1946.

Bailey, S.M., Barth, C.A., Solomon, S.C. A model of nitric oxide in the lower thermosphere. J. Geophys. Res. 107, A10, doi: 10.1029/2001JA000258, 2002.

Bethge, C., Peter, H., Kentischer, T., Halbgewachs, C., Elmore, D., Beck, C. The Chromospheric Telescope, A\&A 534, A105, doi:http://dx.doi.org/10.1051/00046361/201117456, 2011. 
Bolduc, C., Charbonneau, P., Dumoulin, V., Bourqui, M.-S., Crouch, A.-D. A Fast Model for the Reconstruction of Solar Spectral Irradiance in the Near- and Mid-Ultraviolet. Sol. Phys., vol. 279, no. 2, 383-409, doi:10.1007/s11207-012-0019-4, 2012.

Bovensmann, H, Burrows, J. P., Buchwitz, M., Frerick, J., Noel, S., Rozanof, V. V., Chance, K. V., Goede, A. P. H. J. SCIAMACHY: Mission Objective and Measuremetn Modes, Atm. Sci. 56, 127-150, doi:10.1175/1520-0469, 1999.

Brueckner, G. E., Edlow, K. L., Floyd, L. E., Lean, J. L., VanHoosier, M. E. The solar ultraviolet spectral irradiance monitor (SUSIM) experiment onboard the Upper Atmosphere Research Satellite (UARS). ,J. Geophys. Res., vol. 98, no. D6, 1069510711, doi:1020/93JD0041, 1993.

Cebula, R.-P., DeLand, M.-T., Schlesinger, B.-M. Estimates of solar variability using the solar backscatter ultraviolet (SBUV) $2 \mathrm{Mg}$ II index from the NOAA 9 satellite. J. Geophys. Res., vol. 97, no. D11, 11613-11620, doi:10.1029/92JD00893, 1992.

Cessateur, G., Dudok de Wit, T., Kretzschmar, M., Lilensten, J., Hochedez, J.-F., Snow, M. Monitoring the solar UV irradiance spectrum from the observation of a few passbands. Astron. Astrophys., 528, A68, http://dx.doi.org/10.1051/0004-6361/201015903, 2011.

Cessateur, G, Lilensten, J, Barthelemy M. Photoabsorption in Ganymede's atmosphere. Icarus, vol. 218, Issue 1, 308-319, doi:10.1016/j.icarus.2011.11.025, 2012a.

Cessateur, G, Lilensten, J, Dudok de Wit, T. New observation strategies for the solar UV spectral irradiance. J. Space Weather Space Clim., 2, A16. http://dx.doi.org/10.1051/swsc/2012016,2012b.

Cessateur, G., Shapiro, A. I., Yeo, K. L., Krivova, N. A., Tagirov, R., Adams, W., Schmutz, W. Solar spectral irradiance variations on rotation time scales: Comparison between observations and the COSIR model. Astron. Astrophys., submitted, 2015.

Chamberlin, P. C., Woods, T. N., Eparvier, F. G., and Jones, A. R. Next Generation X-Ray Sensor (XRS) for GOES-R Satellite Series. SPIE Proc., 7438, 23, 2009.

Ciraolo, L., Azpilicueta, F., Brunini, C. Meza, A., Radicella, S. M. Calibration errors on experimental slant total electron content (TEC) determined with GPS, Journal of Geodesy, 81-2, 111-120, doi:10.10007/s00190-006-0093-1, 2007.

Davies, K., Degenhardt, W., Hartmann, G.K., Jakowski, N., Leitinger R. Relationships between ionospheric electron content and solar fluxes. Proc. Int. Beacon Sat. Symp. (Ed.: Min-Chang Lee), MIT, Cambridge, 6-10 July, 1992, 188-191, 1992.

DeLand, M., S. Marchenko, The solar chromospheric Ca and Mg indices from Aura OMI, J. Geophys. Res. Atmos., 118, 3415-3423, doi:10.1002/jgrd.50310, 2013.

Didkovsky, L., Judge, D., Wieman, S., Woods, T., Jones, A. EUV SpectroPhotometer (ESP) in Extreme Ultraviolet Variability Experiment (EVE): Algorithms and Calibrations. Sol. Phys., vol. 275, Issue 1-2, 179-205, doi:10.1007/s11207-009-9485-8, 2012.

Domingo, V., Ermolli, I., Fox, P., Fröhlich, C., Haberreiter, M., Krivova, N., Kopp, G., Schmjútz, W., Solanki, S., K., Spruiut, H. C., Unruh, Y., Vögler, A. Solar Surface Magnetism and Irradiance on Time Scales from Days to the 11-Year Cycle, Space Sci. Rev., 145, 337-380, doi:10.1007/s11214-009-9562-1, 2009. 
Dudok de Wit, T., Lilensten, J., Aboudarham, J., Aamblard, P.-O., Kretzschmar, M. Retrieving the solar EUV spectrum from a reduced set of spectral lines. Ann. Geophys. 23, 3055-3069, doi:10.5194/angeo-23-3055-2005, 2005.

Dudok de Wit, T., Bruinsma, S., and Shibasaki, K. Synoptic radio observations as proxies for upper atmosphere modelling. Journal of Space Weather and Space Climate, 4, A06, 2014.

Emmert, J. T., Lean, J. L., Picone, J. M. Record-low thermospheric density during the 2008 solar minimum. Geophys . Res. Lett., 37, L12102, doi:10.1029/2010GL043671, 2010.

Eparvier, F. G., Jones, A. R., Chamberlin, P. C., Woods, T. N., McClintock, W. E., Snow, M. The Extreme Ultraviolet Sensor (EUVS) for GOES-R. SPIE Proc., 7438-19, Bibcode:2009SPIE.7438E..02E, 2009.

Evans, J. S., Strickland, D. J., Woo, W. K., McMullin, D. R., Plunkett, S. P., Viereck, R. A., Hill, S. M., Woods, T. N., Eparvier, F. G. Early Observations by the GOES-13 Solar Extreme Ultraviolet Sensor (EUVS). Solar Phys. 272, 71-115, doi:10.1007/s11207-0099491-x, 2010.

Heelis, R. A., Coley, W. R., Burrell, A. G., Hairston, M. R., Earle, G. D., Perdue, M. D., Power, R. A., Harmon, L. L., Holt, B. J., Lippincott, C. R. Behavior of the $\mathrm{O}^{+} / \mathrm{H}^{+}$ transition height during the extreme solar minimum of 2008. Geophys. Res. Lett., 36, L00C03, doi:10.1029/2009GL038652, 2009.

Huba, J., R. Schunk, G. Khazanov, editors, Modeling the Ionosphere-Thermosphere System, AGU Geophysical Monograph Series, 201, doi:10.1002/9781118704417, 2014.

Gottwald, A., Richter, M., Shaw, P.-S., Li, Z., Arp, U. Bilateral NIST-PTB comparison of spectral Responsivity in the VUV, Metrologia, 48, 02001, doi:10.1088/00261394/48/1A/02001, 2011.

Haberreiter, M. Solar EUV Spectrum Calculated for Quiet Sun Conditions. Sol. Phys., vol. 274, no. 1-2, 473-479, doi:10.1007/s11207-011-9767-9, 2011.

Haberreiter, M., Delouille, V., Mampaey, B., Verbeeck, C., Del Zanna, G., Wieman, S. Reconstruction of the solar EUV irradiance from 1996 to 2010 based on SOHO/EIT images. J. Space Weath. Space Clim., 4, A30, doi:10.1051/swsc/2014027, 2014.

Haberreiter, M., Schmutz, W., Hubeny, I. NLTE model calculations for the solar atmosphere with an iterative treatment of opacity distribution functions. Astron. Astrophys., 492, 833-840, doi:10.1051/0004-6361:200809503, 2008.

Hammer, R., Schleicher, H. Connections between X, EUV/UV and VIS solar activity. Phys. Chem. Earth C 25, 409-412, doi:10.1016/S1464-1917(00)00045-3, 2000.

Harder, J. W., Fontenla, J., Lawrence, G., Woods, T. N., Rottman, G. Solar Phys. 230, 1-2, 169-204, doi:10.1007/s11207-005-1528-1, 2005a.

Harder, J. W., Lawrence, G., Fontenla, J., Rottman, G., Woods, T. N. Solar Phys. 230, 1-2, 141-167, doi:10.1007/s11207-005-5007-5, 2005b.

Harder, J.-W., Thuillier, G., Richard, E.-C., Brown, S.-W., Lykke, K.-R., Snow, M., McClintock, W.-E., Fontenla, J.-M., Woods, T.-N., Pilewskie, P. The SORCE SIM Solar Spectrum: Comparison with Recent Observations. Sol. Phys., vol. 263, 3-27, 
doi:10.1007/s11207-010-9555-y, 2010.

Hinteregger, H. E. Representations of solar EUV fluxes for aeronomical applications. Adv. Space Res. 1, no. 12, 39-52, doi:10.1016/0273-1177(81)90416-6, 1981.

Hinteregger, H. E., Fukui, K., Gilson, G. R. Observational, reference and model data on solar EUV, from measurements on AE-E. Geophys. Res. Lett. 8, 1147-1150, doi:10.1029/GL008i011p01147, 1981.

Hinteregger, H.-E. Far Ultraviolet Radiation, Radiant Flux Density, Satellite Observation, Solar Cycles, Solar Radiation, Radiation Distribution, Spaceborne Astronomy, Tables (Data). Geophys. Res. Let., vol. 4, 231-234, doi:10.1029/GL0041006p00231, 1977.

Hochedez, J.-F., Schmutz, W., Nesladek, M., Stockman, Y., Schühle, U., BenMoussa,, A., Koller, S., Haenen, K., Berghmans, D., Defise, J.-M., Halain, J.-P., Theissen, A., Delouille, V., Slemzin, V., Gillota,y D., Fussen, D., Dominique, M., Vanhellemont, F., McMullin, D., Kretschmar, M., Mitrofanov, A., Nicula, B., Wauters, L., Roth, H., Rozanov, E., Rüedi, I., Wehrli, C., Amano, H., Van der Linden, R., Zhukov, A., Clette, F., Koizumi, S., Mortet, V., Remes, Z., Petersen, R., D'Olieslaeger, M., Roggen, J., Rochus, P. LYRA: a solar UV radiometer on Proba2. Adv. Space Res. 37, 303-312, doi: 10.1016/j.asr.2005.10.041, 2006.

Hoque, M. M., Jakowski, N. A new global empirical NmF2 model for operational use in radio system. Radio Sci., 46, RS6015, doi:10.1029/2011RS004807, 2011.

Jakowski, N., Fichtelmann, B., Jungstand, A. Solar activity control of ionospheric and thermospheric processes. J. Atmos. Terr. Phys., 53, 1125-1130, bibcode: 1991JATP...53.1125J, 1991.

Jakowski, N., Hoque, M., Mayer, C. A new global TEC model for estimating transionospheric radio wave propagation errors. Journal of Geodesy, 85, 965-974, doi:10.1007/s00190011-0455-1, 2011.

Jakowski, N., Heise, S., Wehrenpfennig, A., Schlueter, S., Reimer, R. GPS/GLONASS-based TEC measurements as a contributor for space weather forecast. Journal of Atmospheric and Solar Terrestrial Physics 64, 729-735, doi:10.1016/S1364-6826(02)00034-2, 2002.

Jakowski, N., Mayer, C., Hoque, M. M., Wilken, V. TEC Models And Their Use In Ionosphere Monitoring. Radio Sci., 46, RS0D18, doi:10.1029/2010RS004620, 2011.

Jakowski, N., Wehrenpfennig, A., Heise, S., Reigber, Ch., Luehr, H. GPS Radio Occultation Measurements of the Ionosphere on CHAMP: Early Results. Geophysical Research Letters, 29, No. 10, 951-954, doi:10.1029/2001GL014364, 2002.

Kotov, Yu. D., Kochemasov, A. V., Glyanenko, A. S., Yurov, V. N., and Arkhangelsky, A. I. PHOKA experiment: Description of the equipment and first results. Solar System Res. 45, 153-161, doi:10.1134/S0038094611020109, 2011.

Krivova, N.-A., Vieira, L.-E.-A., Solanki, S.-K. Reconstruction of solar spectral irradiance since the Maunder minimum. J. Geophys. Res., vol. 115, no. A12, doi:10.1029/2010JA015431, 2010.

Kudryashev, G.S., Avakyan, S.V. Ionization and excitation of the upper atmosphere during solar flares. Phys. Chem. Earth (C) 25, 5-6, 511-514, doi:10.1016/S1464- 
1917(00)00067-2, 2000.

Lean, J. Evolution of the Sun's Spectral Irradiance since the Maunder Minimum. Geophys. Res. Lett., vol. 27, no. 16, 2425-2428, doi:10.1029/2000GL000043, 2000.

Lean, J. L., Woods, T. N., Eparvier, F., Meier, R. R., Strickland, D. J. J. Geophys. Res. 116, A01102. doi:10.1029/2010JA015901, 2011.

Lee, Choon-Ki, Han, Shin-Chan, Bilitza, D., Seo, Ki-Weon. Global characteristics of the correlation and time lag between solar and ionospheric parameters in the 27-day period. 77, 219-224, doi:10.1016/j.jastp.2012.01.010, 2012.

Marchenko, S. V., M. T. DeLand. Solar spectral irradiance changes during cycle 24. Ap. J. 789, Issue 2, 117, doi:10.1088/0004-637X/789/2/117, 2014.

McClintock, W. E., G. J. Rottman, and T. N. Woods, Solar Stellar Irradiance Comparison Experiment II (SOLSTICE II): Instrument concept and design, Solar Physics, vol. 203, 225-258, doi:10.1007/s11207-005-7432-x, 2005a.

McClintock, W. E., M. Snow, and T. N. Woods, Solar Stellar Irradiance Comparison Experiment II (SOLSTICE II): Pre-launch and on-orbit calibrations, Solar Physics, vol. 203, 259-294, doi:10.1007/s11207-005-1585-5, 2005b.

Pflug, K., Kotov, Yu. D., Schmidtke, G. EUV-PHOKA measurements of solar spectral irradiance variations in the EUV/XUV region. J. Moscow Phys. Soc. 6, 337-346, 1996.

Rottman, G. J., Woods, T. N., Sparn, T. P. Solar-Stellar Irradiance Comparison Experiment 1. I - Instrument design and operation. J. Geophys. Res. 98, 10667-10677, doi:10.1029/93JD00462, 1993.

Rottman, G. Variations of solar ultraviolet irradiance observed by the UARS SOLSTICE 1991 to 1999. Sp. Sci. Rev., 94, Issue 1/2, 83-91. doi:10.1023/A:1026786315718, 2000.

Rottman, G. J. The SORCE Mission. Solar Phys. 230, Issue 1-2, 7-25. doi:10.1007/s11207005-8112-6, 2005.

Samson, A. R., and Haddad, G. N. Absolute photon flux measurements in the vacuum ultraviolet. J. Opt. Soc. America 64, 47-54, doi:10.1364/JOSA.64.000047,1974.

Samson, A. R. Techniques of Vacuum Ultraviolet Spectroscopy, Wiley, New York, 1967.

Schmidtke, G., Schweizer, W., and Knothe, M. The AEROS-EUV Spectrometer, J. Geophys., vol. 40, 577-584, 1974.

Schmidtke, G., Knothe, M., Heidinger, F. Magnetic electron multiplier: efficiency changes, Appl. Opt., vol. 14, 1645-1648, doi:10.1364/AO.14.001645, 1975.

Schmidtke, G. Solar XUV Measurements with In-Flight Calibration needed for Aeronomy, Space Res., vol. XVI, 229-233, doi:10.1029/GL003i010p00573, 1976a

Schmidtke, G. EUV Indices for Solar-Terrestrial Relations. Geophys. Res. Lett. 3, 573-576, doi:10.1029/GL003i010p00573, 1976 b.

Schmidtke, G., Börsken, N., and Sünder, G. Variability of Solar EUV Fluxes and Exospheric Temperatures, J. Geophys., vol. 49, 146-148, 1981. 
Schmidtke, G. Modelling of the Solar Extreme Ultraviolet Irradiance for Aeronomic Applications, Encyclopedia of Physics, vol. XLIX/7, Geophysics III, part VII, 1-56, ISBN: 3-540-11425-4, 1984.

Schmidtke, G., Seidl, P., Wita, C. Airglow-solar spectrometer instrument (20-700) nm aboard the San Marco D/L satellite. Applied Optics 24, 3206-3213, doi:10.1364/AO.24.003206, 1985.

Schmidtke, G., Doll, H. G., Wita, C., and Chakrabarti, S. Solar EUV/UV and equatorial airglow measurements from San Marco-5. J. Atmospheric Terrestrial Phys. 53 (8), 781785, Bibcode:1991JATP...53..781S, 1991.

Schmidtke, G., Woods, T. N., Worden, J., Rottman, G., Doll, H., Wita, C., Solomon, S. C. Solar Irradiance from the San Marco ASSI: A reference spectrum. Geophys. Res. Lett. 21, 2175-2178, doi:10.1029/92GL02183, 1992.

Schmidtke, G., Doll, H. G., and Wita, C. Measurement of solar EUV/UV radiation of the steeply rising solar cycle 22 during the San Marco 5 mission and proposed instrumentation to achieve high radiometric accuracy. Adv. Space Res. 13, 221-246, doi:10.1016/0273-1177(93)90073-K, 1993.

Schmidtke, G., Doll, H., Seidl, P., Wita, C., Römer, M., Enninghorst, K., Tai, H., Woods, T. N., Worden, J. ASSI-System-Analyse und Koordinierung der Auswertung der Messdaten, Final Report, University of Bonn, Astronom. Institutes, 1995.

Schmidtke, G., Eparvier, F. C., Solomon, S. C., Tobiska, W. K., Woods, T. N. The TIGER (thermospheric-ionospheric geospheric research) program: Introduction. Adv. Space Res. 37, 194-198, doi:10.1016/j.asr.2005.02.088, 2006a.

Schmidtke, G., Brunner, R., Eberhard, D., Halford, B., Klocke, U., Knothe, M., Konz, W., Riedel, W. J., Wolf, H. Sol-ACES: Auto-Calibrating EUV/UV Spectrometers for measurements onboard the International Space Station. Adv. Space Res. 37, 273-282, doi:10.1016/j.asr.2005.01.112, 2006 b.

Schmidtke, G., Nikutowski, B., Jacobi, Ch., Brunner, R., Erhardt, Ch., Knecht, S., Scherle, J., Schlagenhauf, J. Solar EUV Irradiance Measurements by the Auto-Calibrating EUV Spectrometers (SolACES) Aboard the International Space Station (ISS). Sol. Phys., vol. 289, no. 5, 1863-1883, doi:10.1007/s11207-013-0430-5, 2014.

Schmidtke, G. Extreme ultraviolet spectral irradiance measurements since 1946. Hist. Geo Space Sci., 6, 3-22, doi:10.5194/hgss-6-3-2015, 2015.

Schmutz, W., Fehlmann, A., Hüsen, G., Meindl, P., Winkler, R., Thuillier, G., Blattner, P., Buisson, F., Egorova, T., Finsterle, W., Fox, N., Gröbner, J., Hochedez, J. F., Koller, S., Meftah, M., Meisonnier, M., Nyeki, S., Pfiffner, D., Roth, H., Rozanov, E., Spescha, M., Wehrli, C., Werner, L., Wyss, J. U. The PREMOS/PICARD instrument calibration. Metrologia, 46, S202. doi:10.1088/0026-1394/46/4/S13, 2009.

Scholze, F., Vest, R., Saito, T. Report on the CCPR Pilot Comparison: Spectral Responsivity $10 \mathrm{~nm}$ to $20 \mathrm{~nm}$, Metrologia, 47, 02010, doi:10.1088/0026-1394/47/1A/02001.

Schöll, M., Dudok de Wit, T., Kretzschmar, M., and Haberreiter, M., 2015, Making of a solar spectral irradiance dataset: data and methods. J. Space Weath. Space Clim., under review, 2015. 
Shapiro, A. I., Schmutz, W., Schöll, M., Haberreiter, M., Rozanov, E. NLTE solar irradiance modeling with the COSI code. Astron. Astrophys. 517, A48, 13-38, doi:10.1051/00046361/200913987, 2010.

Snow, M., Weber, M., Machol, J., Viereck, R., Richard, E. J. Space Weather Space Climate, 4, A04-1, doi:10.1051/swsc/2014001, 2014.

Solomon, S. C., Bailey, S. M., Woods, T. N. Effect of solar soft X rays in the lower ionosphere. Geophys, Res. Lett., 28, 2149-2152, doi:10.1029/1999GL900236, 2001.

Solomon, S. C., Qian, L. Solar extreme-ultraviolet irradiance for general circulation models. J. Geophys. Res., 110, A10306, doi:10.1029/2005JA011160, 2005.

Solomon, S. C., Woods, T. N., Didkovsky, L. V., Emmert, J. T., Qian L. Anomalously low solar extreme-ultraviolet irradiance and thermospheric density during solar minimum. Geophys. Res. Lett., 37, L16103, doi:10.1029/2010GL044468, 2010.

Solomon, S. C., Qian, L., Didkovsky, L. V., Viereck, R. A., Woods, T. N. Causes of low thermospheric density during the 2007-2009 solar minimum. J. Geophys. Res., 116, A00H07, doi:10.1029/2011JA016508, 2011,

Solomon, S. C., Qian, L., Burns, A. G. The anomalous ionosphere between solar cycles 23 and 24. J. Geophys. Res. Space Physics, 118, 6524-6535, doi:10.1002/jgra.50561, 2013.

Thuillier, G., Floyd., L., Woods, T.-N., Cebula, R., Hilsenrath, E., Hersé, M., Labs, D. In Solar Variability and its Effect on Climate, AGU monograph 141, eds J. Pap and P. Fox, p. 171. Bibliographic Code:2004GMS...141..171T, 2004.

Thuillier, G., Foujols, T., Bolsée D., Gillotay, D., Hersé, M., Peetermans, W., Decuyper, W., Mandel, H., Sperfeld, P., Pape, S., Taubert, D. R., Hartmann, J. SOLAR/SOLSPEC: Scientific Objectives, Instrument Performance and Its Absolute Calibration Using a Blackbody as Primary Standard Source. Sol. Phys., vol. 257, no. 1, 185-213, doi:10.1007/s11207-009-9361-6, 2009.

Thuillier, G., DeLand, M., Shapiro, A., Schmutz, W., Bolsée, D., Melo, S.M.L. The solar spectral irradiance as a function of the $\mathrm{Mg}$ II index for atmosphere and climate modeling. Sol. Phys., vol. 277, no. 2, 245-266, doi:10.1007/s11207-011-9912-5, 2012.

Thuillier, G., Melo' S.-M.-L., Lean, J., Krivova, N.-A., Bolduc, C., Fomichev, V. I., Charbonneau, P., Shapiro, A. I., Schmutz, W., Bolsée, D. Analysis of Different Solar Spectral Irradiance Reconstructions and Their Impact on Solar Heating Rates. Sol. Phys., vol. 289, no. 4, 1115-1142, doi:10.1007/s11207-013-0381-x, 2013.

Thuillier, G., Bolsée, D., Schmidtke, G., Foujols, Th., Nikutowski, B., Brunner, R., Erhardt, Ch., Shapiro, A. V., Schmutz, W., Hersé, M., Gillotay, D., Mandel, H., Petermanns, W., Decuyper, W. The Solar Irradiance Spectrum at Solar Activity Minimum Between Solar Cycles 23 and 24. Sol. Phys., vol. 289, no. 6, 1931-1958, doi:10.1007/s11207-0130461-y, 2014a.

Thuillier, G., Schmidtke, G., Erhardt, Ch., Nikutowski, B., Shapiro, A. I., Bolduc, C., Lean, J., Krivova, N., Charbonneau, P., Cessateur, G., Haberreiter, M., Melo, S., Delouille, V., Mampeay, B., Yeo, K. L., and Schmutz, W. Solar Spectral Irradiance Variability Simultaneously Observed From the International Space Station and by Several 
Instruments in November/December 2012 and Its Modeling, in press in Sol. Phys. Vol. 289, 4433-4452, doi:10.1007/s11207-014-0588-5, 2014b.

Tobiska, W. K., Bouwer, S. D., Bowman, B. R. The development of new solar indices for use in thermospheric density modeling, J. Atmos. Solar-Terr. Phys., vol. 70, no. 5, 803-819, doi:10.1016/j.jastp.2007.11.001, 2008.

Unglaub, C., Jacobi, Ch., Schmidtke, G., Nikutowski, B., Brunner, R. EUV-TEC proxy to describe ionospheric variability using satellite-borne solar EUV measurements: first results. Adv. Space Res. 47, 1578-1584, doi:10.1016/j.asr.2010.12.014, 2011.

Vanhoosier, M.-E., Bartoe, J.-D.-F., Brueckner, G., Prinz, D.,-K. Results from the Solar Ultreviolet Spectral Irradiance Monitor flown on SpaceLab 2. Bulletin of the American Astronomical Society, vol. 27, no. 3, 163-168, bibcode: 1988ApL\&C..27..163V, 1988.

Wieman, S. R., Didkovsky, L. V., Judge, D. SOHO/CELIAS EUV Monitor (SEM) Absolute Solar EUV Irradiance Measurements Spanning Two Solar Minima, American Geophysical Union, Fall Meeting 2010, bibcode: 2010AGUFMGC33C..05W, 2010.

Woods, T. N., and Rottman G. J. Solar EUV irradiance derived from a sounding rocket experiment on November 10, 1988, J. Geophys. Res. 95, 6227-6263, doi:10.1029/JA095iA05p06227, 1990.

Woods, T. N., Bailey, S. M., Eparvier, F. G., Lawrence, G., Lean, J., McClintock, B., Roble, R., Rottman, G. J., Solomon, S. C., Tobiska, W. K., White, O. R. TIMED solar EUV experiment. Phys. Chem. Earth C 25, 393-396, doi: 10.1016/S1464-1917(00)00040-4, 2000.

Woods, T. N., Eparvier, F.-G., Bailey, S.-M., Chamberlin, Ph.-C., Lean, J., Rottman, G.-J., Solomon, S.-C., Tobiska, W.-K., Woodraska, D.-L. Solar EUV Experiment (SEE): Mission overview and first results. J. Geophys. Res., vol. 110, no. A1, 115-144, doi:10.1029/2004JA010765, 2005.

Woods, T. N.: Recent advances in observations and modeling of solar ultraviolet and x-ray spectral irradiance, Adv. Space Res., 42, 895-902, doi:10.1016/j.asr.2007.09.026, 2008.

Woods, T.- N., Chamberlin, P. C., Harder, J. W., Hock, R., A., Snow, M., Eparvier, F. G., Fontenla, J., McClintock, W. E., Richard, E. C. Solar Irradiance Reference Spectra (SIRS) for the 2008 Whole Heliosphere Interval (WHI), Geophys. Res. Let., vol. 36, 1. L01101, doi:10.1029/2008GL036373, 2009.

Woods, T.-N., Eparvier, F.-G., Hock, R., Jones, A. R., Woodraska, D., Judge, D., Didkovsky, L., Lean, J., Mariska, J., Warren, H., McMullin, D., Chamberlin, P., Berthiaume, G., Bailey, S., Fuller-Rowell, T., Sojka, J., Tobiska, W.-K., Viereck, R. Extreme Ultraviolet Experiment (EVE) on the Solar Dynamic Observatory (SDO). Sol. Phys., 275, no. 1-2, 115-144. doi:10.1007/s11207-009-9487-6, 2010.

Worden, J., and Woods, T. N.: Private communication, 1996.

Worden, J., Woods, T. N., Rottman, G. J., Schmidtke, G., Tai, H., Doll, H. G., Solomon, S. C. Calibration of the San Marco Airglow-Solar Spectrometer Instrument in the extreme ultraviolet, Opt. Eng. 35, 554-563, doi: 10.1117/1.601037, 1996. 